\title{
The bispectrum of relativistic galaxy number counts
}

\author{
Enea Di Dio ${ }^{a, b, c}$, Ruth Durrer ${ }^{c}$, Giovanni Marozzi ${ }^{c}$, Francesco \\ Montanari ${ }^{c, d}$
}

\author{
${ }^{a}$ Osservatorio Astronomico di Trieste, Università degli Studi di Trieste, Via Tiepolo 11, \\ 34143 Trieste, Italy \\ ${ }^{b}$ INFN, Sezione di Trieste, Via Valerio 2, I-34127 Trieste, Italy \\ ${ }^{c}$ Université de Genève, Département de Physique Théorique and CAP, 24 quai Ernest- \\ Ansermet, CH-1211 Genève 4, Switzerland \\ ${ }^{d}$ Physics Department, University of Helsinki and Helsinki Institute of Physics \\ P.O. Box 64, FIN-00014, University of Helsinki, Finland \\ E-mail: Enea.DiDio@oats.inaf.it, Ruth.Durrer@unige.ch, \\ Giovanni.Marozzi@unige.ch, Francesco.Montanari@helsinki.fi
}

\begin{abstract}
We discuss the dominant terms of the relativistic galaxy number counts to second order in cosmological perturbation theory on sub-Hubble scales and on intermediate to large redshifts. In particular, we determine their contribution to the bispectrum. In addition to the terms already known from Newtonian second order perturbation theory, we find that there are a series of additional 'lensing-like' terms which contribute to the bispectrum. We derive analytical expressions for the full leading order bispectrum and we evaluate it numerically for different configurations, indicating how they can be measured with upcoming surveys. In particular, the new 'lensing-like' terms are not negligible for wide redshift bins and even dominate the bispectrum at well separated redshifts. This offers us the possibility to measure them in future surveys.
\end{abstract}




\section{Contents}

1 Introduction $\quad 2$

2 Galaxy Number Counts 3

2.1 Basic ideas 3

2.2 Second order number counts: the dominant terms 4

2.3 Comparison with the Newtonian calculation 8

3 The dominant terms in the bispectrum $\quad 10$

3.1 The bispectrum 10

3.2 The Newtonian terms 12

$\begin{array}{lll}3.3 & \text { Terms containing lensing } & 14\end{array}$

3.4 Bispectrum for the second order velocity and lensing terms $\quad 15$

$\begin{array}{lll}3.4 .1 & \text { Second order redshift space distortion } & 16\end{array}$

$\begin{array}{ll}3.4 .2 \text { Second order lensing } & 18\end{array}$

$\begin{array}{ll}\text { 3.4.3 Comparison with the second order density } & 19\end{array}$

$4 \quad$ Numerical examples and how to measure lensing terms 22

$\begin{array}{lll}4.1 & \text { Window functions } & 27\end{array}$

5 Conclusions $\quad 30$

A Derivation of bispectrum terms

A.1 Density term 32

A.1.1 Monopole $\quad 35$

$\begin{array}{lll}\text { A.1.2 Dipole } & 35\end{array}$

$\begin{array}{lll}\text { A.1.3 Quadrupole } & 35\end{array}$

A.2 Term $\mathcal{H}^{-2} \partial_{r} v \partial_{r}^{3} v \quad 36$

A.3 Term $\mathcal{H}^{-2} \partial_{r}^{2} v \partial_{r}^{2} v \quad 37$

A.4 Term $\mathcal{H}^{-1} \partial_{r} v \partial_{r} \delta \quad 38$

$\begin{array}{lll}\text { A.5 Term } \mathcal{H}^{-1} \partial_{r}^{2} v \delta & 39\end{array}$

$\begin{array}{llr}\text { A.6 } & \text { Term } \mathcal{H}^{-1} \partial_{r}^{2} v \kappa & 39\end{array}$

A.7 Term $\kappa \delta \quad 40$

A.8 Term $\kappa^{2} \quad 40$

A.9 Term $\nabla_{a} \delta \nabla^{a} \psi \quad 42$

A.10 Term $\mathcal{H}^{-1} \nabla_{a} \partial_{r}^{2} v \nabla^{a} \psi$

A.11 Term $\nabla_{b} \kappa \nabla^{b} \psi$

A.12 Term $\int_{0}^{r(z)} \frac{d r}{r} \nabla^{b} \kappa \nabla_{b} \Psi_{1}$

A.13 Term $\int_{0}^{r_{s}} d r \frac{r_{s}-r}{r_{s} r} \Delta_{2}\left(\nabla^{b} \Psi_{1} \nabla_{b} \Psi_{1}\right) \quad 49$

B Magnification Bias $\quad 53$

C Intensity mapping $\quad 54$ 


\section{Introduction}

A present challenge in cosmology is to repeat the big success of CMB observations, see e.g. [1, $2]$, with large galaxy surveys which are planned and underway [3-7]. The advantage is that galaxies fill 3-dimensional space as compared to the 2-dimensional surface of last scattering, which significantly increases the number of independent modes that can be observed. This is especially important if we want to measure not only the power spectrum (the harmonic transform of the 2-point function) but also the bispectrum (the harmonic transform of the 3 -point function).

Primordial non-Gaussianities generated during inflation [8,9] contain information about physical interactions at inflationary energy scales. However, gravity is itself non-linear and therefore also evolution under gravity after inflation introduces non-Gaussianities even if primordial fluctuations are Gaussian. It is important to distinguish these gravitational nonGaussianities from the primordial ones. In order to do so, one has to calculate the bispectrum induced at second order in perturbation theory. This has been carried out within Newtonian gravity in great detail, see [10] for a review.

In a fully relativistic analysis, we take into account that observations are made on the past light-cone. We cannot observe a constant time hypersurface, but we observe directions and redshifts of galaxies. We then can observe the fluctuation of their number within a small opening angle around a given direction $\mathbf{n}$ and within a redshift bin around an observed redshift $z$. But also the observed direction and redshift are perturbed. This relativistic number counts have been determined to first order in perturbation theory in [11-14]. Here we follow especially the latter two references where the perturbation of the number counts, $\Delta(\mathbf{n}, z)$ is consistently considered as a function of observed direction $\mathbf{n}$ and redshift $z$. These are good coordinates on the past light-cone since they are directly observable. In a previous paper, Ref. [15], we have derived the number counts to second order following a purely geometric approach based on the so-called Geodesic Light-Cone (GLC) coordinates [16] and using the result for the luminosity distance obtained in [17-19]. Results for the second order number counts are also published in [20-22], and in [23] the relativistic bispectrum is computed in the squeezed limit (see also [24]). In [15] we have identified the terms which dominate at sub-Hubble scales and at intermediate to large redshifts. These are quadratic terms which contain in total four transverse derivatives of the Bardeen potentials. Parametrically they are of the same amplitude as the square of density perturbations which means that they can become large.

In [15] we have computed the bispectrum from only a few of these dominant terms. In this paper we complete the previous analysis. We calculate the bispectrum from all the dominant terms. We also compare our dominant terms with the ones obtained in the Newtonian analysis and find that, apart from the lensing-like terms which are new, they fully agree. The main difference is that we express the bispectrum, including the 'Newtonian terms', in the directly observable angular and redshift space. Finally, we compare the new lensing terms with the standard Newtonian terms and device a strategy to measure them.

The paper is organized as follows. In the next section we present the dominant terms of the second order number counts, interpret the different terms and compare our expression with previous Newtonian results. In Section 3 we derive expressions for their contribution to the bispectrum. In Section 4 we then show numerical examples of all the leading contributions to the bispectrum, and discuss the situations in which the lensing-like terms can be non negligible and even dominate the signal. In Section 5 we conclude. Some lengthy derivations 
are deferred to Appendix A. We also derive second order expressions for the magnification bias in Appendix B and for intensity mapping in Appendix C.

\section{Galaxy Number Counts}

\subsection{Basic ideas}

In galaxy surveys we observe the number $N$ of galaxies in a redshift bin $d z$ and a solid angle $d \Omega$ in terms of the observed redshift $z$ and of the observation direction defined by the unit vector $\mathbf{- n}$ (in this work $\mathbf{n}$ denotes the direction of propagation of the photon). The fluctuation of galaxy number counts $[13,14]$ is defined as a function of these observable coordinates

$$
\Delta(\mathbf{n}, z) \equiv \frac{N(\mathbf{n}, z)-\langle N\rangle(z)}{\langle N\rangle(z)},
$$

where $\langle\cdots\rangle$ denotes the ensemble average over many realisations. In observations this is replaced by an average over observed directions, at a fixed observed redshift.

Traditionally, galaxy density fluctuations have been represented in Fourier space [25]. This has the advantage that in the case of Gaussian fluctuations, the full information is contained in the power spectrum, $P(k)$ and the density fluctuations at different $k$ 's, $\delta\left(\mathbf{k}_{1}\right)$, $\delta\left(\mathbf{k}_{2}\right)$ are, at least theoretically, uncorrelated as a consequence of statistical homogeneity. Correspondingly, the bispectrum is also represented in Fourier space, $B\left(\mathbf{k}_{1}, \mathbf{k}_{2}\right)$. It is a function only of the three variables determining the triangle spanned by $\mathbf{k}_{1}$ and $\mathbf{k}_{2}$. The disadvantage of these variables is the fact that the observed fluctuations live on the backward light-cone and not on an equal time hypersurface on which we might perform a Fourier transform. To move them onto a fixed time hypersurface one must make assumptions about the time evolution of fluctuations. Furthermore, it is not simple to include line of sight deflection effects (lensing) in such a formalism, as lensing mixes different scales. Furthermore, as mentioned above, the truly measured quantities are redshifts and angles in the sky. To convert them to distances we have to assume a cosmological model, i.e. fix the cosmological parameters. It is then somewhat inconsistent to use the matter power spectrum to determine cosmological parameters, e.g., via the baryon acoustic oscillations (BAO), see [26].

Because of these arguments, we prefer to describe clustering in the directly observed angle and redshift space. Doing this, already the power spectrum, $C_{\ell}\left(z_{1}, z_{2}\right)$ depends on three variables, the multipole $\ell$ and two redshifts. As we shall see, the (reduced) bispectrum even depends on six variables, $b_{\ell_{1} \ell_{2} \ell_{3}}\left(z_{1}, z_{2}, z_{3}\right)$. This of course makes the data more noisy and more difficult to analyze. But these spectra also contain much more information. As for the power spectrum in [27], we shall find for the bispectrum in this work, that we can use number counts not only to determine density fluctuations and redshift space distortions, but when correlating different redshifts, they are also very sensitive to the gravitational potential, especially to the lensing potential. Therefore, this more complex treatment has in principle the capacity to test General Relativity by investigating the consistency between the inferred density fluctuations and the lensing potential.

In Ref. [15] we have derived an expression for the number counts for scalar perturbations to second order. The derivation has been performed in the geodesic lightcone (GLC) gauge [16], and then expressed in the flat Poisson gauge (or longitudinal gauge) defined by 
metric perturbations of the form

$$
\begin{aligned}
d s^{2} & =a^{2}\left[-(1+2 \Psi) d \eta^{2}+(1-2 \Phi) \delta_{i j} d x^{i} d x^{j}\right] \\
\Phi & =\Phi^{(1)}+\Phi^{(2)} \\
\Psi & =\Psi^{(1)}+\Psi^{(2)}
\end{aligned}
$$

Here $\Phi$ and $\Psi$ are the gauge invariant Bardeen potentials which can also be defined in any other gauge. Note that several publications expand $\Phi=\Phi^{(1)}+\frac{1}{2} \Phi^{(2)}$, etc. which leads to different factors of 2 in some of the results. Here, following [10], we do not introduce any factor in front of the second order term.

Because the perturbed Einstein equations are not known in GLC gauge, the change to longitudinal gauge is necessary to compare the derived results with current and future surveys. Starting from a relatively simple result in GLC gauge, after the coordinate transformation, the full expression for $\Delta^{(2)}(\mathbf{n}, z)$ obtained in [15] becomes long and cumbersome, filling several pages and we do not want to repeat it here.

However, most of the terms are very small and appreciable only at scales close to the Hubble scale. These terms are most probably not measurable due to cosmic variance. Our aim is to determine the amplitude of all the terms which appear at the same parametrical order in $k / \mathcal{H}$ as the leading Newtonian terms. At second order these are the terms $\propto(k / \mathcal{H})^{4} \Phi^{2} \propto \delta^{2}$. Here $\mathcal{H}$ denotes the comoving Hubble parameter, $k$ is the wavenumber of the perturbation and $\delta$ is the total matter density perturbation.

\subsection{Second order number counts: the dominant terms}

We concentrate here on the 'dominant terms' on intermediate to large redshift and subHubble scales, where the pure Doppler and potential terms can be neglected. These are the terms with the maximal number of spatial derivatives given in Eq. (4.45) of Ref. [15]. All other terms are suppressed by at least one factor $\mathcal{H} / k$ with respect to these terms.

We introduce the Weyl potential and the following integrals

$$
\begin{aligned}
\Phi_{W} & =\frac{1}{2}(\Phi+\Psi) \\
\psi(\mathbf{n}, z) & =-2 \int_{0}^{r(z)} d r \frac{r(z)-r}{r(z) r} \Phi_{W}^{(1)}\left(-r \mathbf{n}, \eta_{0}-r\right), \quad-2 \kappa^{(1)}=\Delta_{2} \psi(\mathbf{n}, z), \\
\Psi_{1}(\mathbf{n}, z) & =\frac{2}{r(z)} \int_{0}^{r(z)} d r \Phi_{W}^{(1)}\left(-r \mathbf{n}, \eta_{0}-r\right) .
\end{aligned}
$$

Here $\eta$ denotes conformal time and $r(z)=\eta_{0}-\eta(z)$ is the conformal distance to a point on the (background) light-cone at redshift $z$. The integral $\psi(\mathbf{n}, z)$ is the (first order) lensing potential and $\kappa^{(1)}$ is the first order magnification or convergence, while $\Psi_{1}(\mathbf{n}, z)$ is the time delay integral, $r(z) \Psi_{1}(\mathbf{n}, z)$ is the Shapiro time delay. $\Delta_{2}$ is the (dimensionless) Laplacian on $\mathbb{S}^{2}$ with respect to the direction $\mathbf{n}$.

The first order number count fluctuations are given by

$$
\begin{aligned}
\Delta^{(1)}(\mathbf{n}, z,)= & \delta^{(1)}+\frac{1}{\mathcal{H}} \partial_{r}^{2} v^{(1)}-2 \kappa^{(1)}+2 \Psi_{1}+\frac{1}{\mathcal{H}} \dot{\Phi}^{(1)}-2 \Phi^{(1)}+\Psi^{(1)} \\
& +\left(\frac{\dot{\mathcal{H}}}{\mathcal{H}^{2}}+\frac{2}{r \mathcal{H}}\right)\left(\Psi^{(1)}+\partial_{r} v^{(1)}+\int_{0}^{r} d \tilde{r}\left(\dot{\Phi}^{(1)}+\dot{\Psi}^{(1)}\right)\right) \\
= & \Sigma^{(1)}+R^{(1)}
\end{aligned}
$$


where $\Sigma^{(n)}$ and $R^{(n)}$ identify the leading and subleading terms of nth order, with the leading terms of order $(k / \mathcal{H})^{2 n} \Phi^{n}$ like the density term, $\delta^{(n)}$. In these expressions we suppress the arguments which are $\mathbf{x}(\mathbf{n}, z)=-r(z) \mathbf{n}$ and $\eta(z)=\eta_{0}-r(z)$. Here $\delta^{(1)}$ is the first order density fluctuation, $v^{(1)}$ is the first order velocity potential so that $v_{r}=-\partial_{r} v$ is the radial component of the velocity.

In this paper we do not include biasing. Neither galaxy nor magnification or evolution bias are considered. This is not a good approximation, but assuming deterministic bias it is not difficult to insert the corresponding factors $b(z), s(z)$ or $f_{\text {evo }}(z)$ for a given survey, see e.g. [27]. In this work we set $b(z)=1$ and $s(z)=f_{\text {evo }}(z)=0$ in all the plots shown. We do, however discuss the modification of the magnification bias $s(z)$ when second order perturbations are considered both in the main text and in Appendix B.

The dominant terms of the first order number counts are the first three terms of Eq. (2.9). They are given by

$$
\Sigma^{(1)}(\mathbf{n}, z)=\delta^{(1)}(-r(z) \mathbf{n}, z)+\mathcal{H}^{-1}(z) \partial_{r}^{2} v^{(1)}(-r(z) \mathbf{n}, z)-2 \kappa^{(1)}(-r(z) \mathbf{n}, z) .
$$

All other terms are suppressed by at least one factor $\mathcal{H} / k$. This is correct for $z \gtrsim 0.2$ where $1 / r(z) \lesssim k$ and we can neglect also the term proportional to $(\mathcal{H} r(z))^{-1}\left(\partial_{r} v^{(1)}+\Psi^{(1)}\right)$ which blows up at very small redshift. The first two terms in (2.9) are the well known density and redshift space distortion (RSD), while the third term is the lensing term which becomes relevant at high redshift. RSD and lensing represent respectively a radial and a transverse volume distortion, see [13].

The second order expression allows an analogous split into leading and sub-leading terms,

$$
\Delta^{(2)}(\mathbf{n}, z,)=\Sigma^{(2)}+R^{(2)}-\left\langle\Sigma^{(2)}+R^{(2)}\right\rangle .
$$

The dominant terms of the number counts at second order can be written in the form $\Sigma^{(2)}-$ $\left\langle\Sigma^{(2)}\right\rangle$.

The definitions (2.6) and (2.7) imply

$$
\begin{aligned}
\frac{d \psi}{d r} & =-\frac{1}{r} \Psi_{1}, \\
\frac{d\left(r \Psi_{1}\right)}{d r} & =2 \Phi_{W}^{(1)} .
\end{aligned}
$$

With the help of these identities one can rewrite the expression given in Eq. (4.45) of $[15]$ in the form

$$
\begin{aligned}
\Sigma^{(2)}(\mathbf{n}, z)= & \delta^{(2)}+\mathcal{H}^{-1} \partial_{r}^{2} v^{(2)}+\mathcal{H}^{-2}\left[\left(\partial_{r}^{2} v\right)^{2}+\partial_{r} v \partial_{r}^{3} v\right]+\mathcal{H}^{-1}\left(\partial_{r} v \partial_{r} \delta+\partial_{r}^{2} v \delta\right) \\
& -2 \delta \kappa+\nabla_{a} \delta \nabla^{a} \psi+\mathcal{H}^{-1}\left[-2\left(\partial_{r}^{2} v\right) \kappa+\nabla_{a}\left(\partial_{r}^{2} v\right) \nabla^{a} \psi\right]-2 \kappa^{(2)}+2 \kappa^{2}-2 \nabla_{b} \kappa \nabla^{b} \psi \\
& -\frac{1}{2 r(z)} \int_{0}^{r(z)} d r \frac{r(z)-r}{r} \Delta_{2}\left(\nabla^{b} \Psi_{1} \nabla_{b} \Psi_{1}\right)-2 \int_{0}^{r(z)} \frac{d r}{r} \nabla^{a} \Psi_{1} \nabla_{a} \kappa .
\end{aligned}
$$

Here the derivatives $\nabla_{a}$ are covariant derivatives with respect to the direction $-\mathbf{n}$ and we have introduced the second order convergence as

$$
\kappa^{(2)}=-\frac{1}{2} \Delta_{2} \psi^{(2)} \quad \text { where } \quad \psi^{(2)}(\mathbf{n}, z)=-\int_{0}^{r(z)} d r \frac{r(z)-r}{r(z) r}(\Psi+\Phi)^{(2)}\left(-r \mathbf{n}, \eta_{0}-r\right) .
$$


Note that our definitions of $\Phi^{(2)}$ and $\Psi^{(2)}$ here differ by a factor 2 from those in Ref. [15]. In order to alleviate the notation we suppress the superscript ${ }^{(1)}$ in all quadratic expressions where it is understood since all our expressions are only correct to second order.

Equation (2.13) is the most important equation of this section. We now give the physical interpretation of all its terms.

First we split it into the Newtonian part given by the first line of (2.13), which contains only density and velocity terms, and the rest which contains lensing given by the second and third lines. The first two terms, $\delta^{(2)}+\mathcal{H}^{-1} \partial_{r}^{2} v^{(2)}$, are simply the second order Newtonian expression, density and redshift space distortion. The fourth and fifth terms can be written as $\mathcal{H}^{-1} \partial_{r} v \partial_{r}\left(\delta+\mathcal{H}^{-1} \partial_{r}^{2} v\right)$. They take into account, by a first order Taylor expansion, that due to the first order RSD we have to evaluate the fluctuation at a slightly different redshift. The third and sixth terms just account for the radial volume distortion by RSD, which now has to be multiplied also by the first order perturbation, $\left(\mathcal{H}^{-1} \partial_{r}^{2} v\right)\left(\delta+\mathcal{H}^{-1} \partial_{r}^{2} v\right)$.

The terms which contain a product of Newtonian and lensing contribution can be interpreted like the Newtonian terms, except that now we consider the transverse displacement in the second and forth terms on the second line, $\left(\nabla^{a} \psi\right) \nabla_{a}\left(\delta+\mathcal{H}^{-1} \partial_{r}^{2} v\right)$, and the transverse volume distortion in the first and the third terms on the second line, $-2 \kappa\left(\delta+\mathcal{H}^{-1} \partial_{r}^{2} v\right)$.

We now show that all the remaining terms, i.e. the 'pure lensing' contributions to $\Sigma^{(2)}$, are simply the second order contribution to the determinant of the Jacobian of the lens map,

$$
\begin{aligned}
A_{a}{ }^{b} & =\nabla_{a} \theta^{b}, \\
\operatorname{det}\left(A_{a}{ }^{b}\right) & =\left(1+\nabla_{1} \delta \theta^{1}\right)\left(1+\nabla_{2} \delta \theta^{2}\right)-\nabla_{1} \delta \theta^{2} \nabla_{2} \delta \theta^{1}
\end{aligned}
$$

Here $\theta^{a}(z, \mathbf{n})=-\mathbf{n}^{a}+\delta \theta^{a}$ is the angular direction of the source as a function of the observed direction (to lowest order these are just the polar angles of $\left.-\mathbf{n}=\left(\theta^{1}, \theta^{2}\right)^{(0)}\right)$.

To calculate this determinant we remember that our definition of $\kappa^{(2)}$ is not the divergence of the second order deflection angle. We define this divergence as $1-\kappa_{\text {mag }}=\nabla_{a} \theta^{a} / 2$. To first order we obtain $\kappa_{\text {mag }}^{(1)}=\kappa^{(1)}$ while to second order the leading term (i.e. the term with four derivatives) for $\kappa_{\text {mag }}$ is given by

$$
\begin{aligned}
\kappa_{\text {mag }}^{(2)}= & \kappa^{(2)}+\nabla_{b} \kappa \nabla^{b} \psi+\frac{1}{4} \int_{0}^{r(z)} d r \frac{r(z)-r}{r(z) r} \Delta_{2}\left(\nabla^{b} \Psi_{1} \nabla_{b} \Psi_{1}\right) \\
& +\int_{0}^{r(z)} \frac{d r}{r} \nabla^{a} \Psi_{1} \nabla_{a} \kappa-\frac{1}{4}\left(\nabla_{b} \nabla_{a} \psi\right)\left(\nabla^{b} \nabla^{a} \psi\right) .
\end{aligned}
$$

This can be obtained from the corresponding leading deflection contribution computed in [28, 29], or from the full result which has been calculated in [17, 29], with the help of the following useful identities

$$
\begin{aligned}
\int_{0}^{r(z)} d r \frac{r(z)-r}{r(z) r} \nabla_{a}\left(\nabla^{a} \nabla^{d} \Phi_{W} \nabla_{d} \psi\right)= & \frac{1}{2} \int_{0}^{r(z)} \frac{d r}{r} \nabla_{a} \Delta_{2} \Psi_{1} \nabla^{a} \psi-\frac{1}{4} \nabla_{a} \nabla_{d} \psi \nabla^{a} \nabla^{d} \psi \\
& +\frac{1}{2} \int_{0}^{r(z)} d r \frac{r(z)-r}{r(z) r} \Delta_{2}\left(\nabla_{a} \Psi_{1} \nabla^{a} \Psi_{1}\right) \\
\int_{0}^{r(z)} \frac{d r}{r} \nabla_{a} \Delta_{2} \Psi_{1} \nabla^{a} \psi= & -\nabla_{a} \Delta_{2} \psi \nabla^{a} \psi-\int_{0}^{r(z)} \frac{d r}{r} \nabla_{a} \Psi_{1} \nabla^{a} \Delta_{2} \psi
\end{aligned}
$$


With Eq. (2.17) the second order terms of (2.16) are simply

$$
\begin{aligned}
\operatorname{det}\left(A_{a}{ }^{b}\right)^{(2)} & =-2 \kappa_{(\mathrm{mag})}^{(2)}+\nabla_{1} \delta \theta^{1} \nabla_{2} \delta \theta^{2}-\nabla_{1} \delta \theta^{2} \nabla_{2} \delta \theta^{1} \\
& =-2 \kappa_{\mathrm{mag}}^{(2)}+2 \kappa^{2}-\frac{1}{2}\left(\nabla_{b} \nabla_{a} \psi\right)\left(\nabla^{b} \nabla^{a} \psi\right) \\
& =\Sigma_{\text {lens }}^{(2)},
\end{aligned}
$$

where $\Sigma_{\text {lens }}^{(2)}$ denotes the last three terms of the second line and the full third line of our expression (2.13), i.e. the pure lensing terms. As one naively expects, these pure lensing contributions also to second order are given by the determinant of the Jacobian of the lens map. Here we neglect vector and tensor perturbations which also appear at second order but are subdominant in our derivative counting. This pure lensing contribution does not quite agree with the corresponding expression in Ref. [20] ${ }^{1}$.

In the remainder of this paper we use Eq. (2.13) to determine the bispectrum of the number counts. For this we first have to express the second order perturbations $\delta^{(2)}, v^{(2)}$ and $\kappa^{(2)}$ in terms of first order quantites.

The second order density fluctuation, $\delta^{(2)}$, velocity potential $v^{(2)}$ and Weyl potential $\Phi_{W}^{(2)}$ are obtained by solving the perturbed Einstein equations to second order, see [30]. The difference of the relativistic solutions from the Newtonian ones are subdominant with respect to our counting, so that we shall use the Newtonian expressions for $\delta^{(2)}, v^{(2)}$ and $\Phi_{W}^{(2)}$ which can be found, e.g., in [10]. In Fourier space ${ }^{2}$ one has

$$
\begin{aligned}
\delta^{(2)}(\mathbf{k}, \eta) & =\frac{1}{(2 \pi)^{3}} \int d^{3} k_{1} d^{3} k_{2} \delta_{D}\left(\mathbf{k}-\mathbf{k}_{1}-\mathbf{k}_{2}\right) F_{2}\left(\mathbf{k}_{1}, \mathbf{k}_{2}\right) \delta\left(\mathbf{k}_{1}, \eta\right) \delta\left(\mathbf{k}_{2}, \eta\right) \\
v^{(2)}(\mathbf{k}, \eta) & =-\frac{\mathcal{H}}{k^{2}} \frac{f^{2}(z)}{(2 \pi)^{3}} \int d^{3} k_{1} d^{3} k_{2} \delta_{D}\left(\mathbf{k}-\mathbf{k}_{1}-\mathbf{k}_{2}\right) G_{2}\left(\mathbf{k}_{1}, \mathbf{k}_{2}\right) \delta\left(\mathbf{k}_{1}, \eta\right) \delta\left(\mathbf{k}_{2}, \eta\right) \\
\Phi_{W}^{(2)}(\mathbf{k}, \eta) & =-\frac{3 \mathcal{H}^{2} \Omega_{m}(\eta)}{2 k^{2}} \delta^{(2)}(\mathbf{k}, \eta) .
\end{aligned}
$$

Here $\delta_{D}$ are Dirac-delta functions, $f(z)=d \log D_{1} / d \log a$ is the growth factor and $D_{1}$ is the linear growth rate of density perturbations. The kernels $F_{2}$ and $G_{2}$ are given by $[10,31]$

$$
\begin{aligned}
F_{2}\left(\mathbf{k}_{1}, \mathbf{k}_{2}\right) & =\frac{5}{7}+\frac{1}{2} \frac{\mathbf{k}_{1} \cdot \mathbf{k}_{2}}{k_{1} k_{2}}\left(\frac{k_{1}}{k_{2}}+\frac{k_{2}}{k_{1}}\right)+\frac{2}{7}\left(\frac{\mathbf{k}_{1} \cdot \mathbf{k}_{2}}{k_{1} k_{2}}\right)^{2} \\
& =\frac{17}{21}+\frac{1}{2}\left(\frac{k_{1}}{k_{2}}+\frac{k_{2}}{k_{1}}\right) P_{1}\left(\hat{\mathbf{k}}_{1} \cdot \hat{\mathbf{k}}_{2}\right)+\frac{4}{21} P_{2}\left(\hat{\mathbf{k}}_{1} \cdot \hat{\mathbf{k}}_{2}\right), \\
G_{2}\left(\mathbf{k}_{1}, \mathbf{k}_{2}\right) & =\frac{3}{7}+\frac{1}{2} \frac{\mathbf{k}_{1} \cdot \mathbf{k}_{2}}{k_{1} k_{2}}\left(\frac{k_{1}}{k_{2}}+\frac{k_{2}}{k_{1}}\right)+\frac{4}{7}\left(\frac{\mathbf{k}_{1} \cdot \mathbf{k}_{2}}{k_{1} k_{2}}\right)^{2} \\
& =\frac{13}{21}+\frac{1}{2}\left(\frac{k_{1}}{k_{2}}+\frac{k_{2}}{k_{1}}\right) P_{1}\left(\hat{\mathbf{k}}_{1} \cdot \hat{\mathbf{k}}_{2}\right)+\frac{8}{21} P_{2}\left(\hat{\mathbf{k}}_{1} \cdot \hat{\mathbf{k}}_{2}\right),
\end{aligned}
$$

\footnotetext{
${ }^{1}$ While the leading terms of Eq. (2.10) in [20] do have the same functional form as our Eq. (2.20), their result for $\kappa_{\text {mag }}^{(2)}$ does not agree with Eq. (2.17). The only leading term present in Eq. (2.12) of [20] for $\kappa_{\text {mag }}^{(2)}$ seems to be our $\kappa^{(2)}$. The other non-trivial leading terms contributing to $\kappa_{\text {mag }}^{(2)}$ are missing.

${ }^{2}$ We use the following Fourier convention

$$
f(\mathbf{x})=\frac{1}{(2 \pi)^{3}} \int d k^{3} \hat{f}(\mathbf{k}) e^{-i \mathbf{k} \cdot \mathbf{x}} .
$$
}

Note that different Fourier notations can lead to a different sign of the velocity terms. 
where $P_{1}$ and $P_{2}$ denote the first and second Legendre polynomials. For more details, see [10].

Note that even though $v^{(2)}$ and $\Phi_{W}^{(2)}$ are suppressed by factors of $\mathcal{H} / k^{2}$ and $\mathcal{H}^{2} / k^{2}$ with respect to $\delta^{(2)}$, our terms are $\mathcal{H}^{-1} \partial_{r}^{2} v^{(2)} \propto\left(k^{2} / \mathcal{H}\right)(\hat{\mathbf{k}} \cdot n)^{2} v^{(2)}$ and $\kappa^{(2)} \sim \ell^{2} \Phi_{W}^{(2)} \sim(k / \mathcal{H})^{2} \Phi_{W}^{(2)}$. Hence $\mathcal{H}^{-1} \partial_{r}^{2} v^{(2)}$ and $\kappa^{(2)}$ are expected to be of the same order as $\delta^{(2)}$. Furthermore, within the Newtonian approximation the potentials $\Phi, \Psi$ and $\Phi_{W}$, all agree and are equal to the Newtonian potential. In a fully relativistic calculation these potentials differ, but the difference is given by the anisotropic stress which is small, especially for cold dark matter (of the order of the kinetic energy) and it does not contribute in our determination of the dominant terms.

\subsection{Comparison with the Newtonian calculation}

It is well-known that the evolution of matter perturbations is correctly described by Newtonian gravity on small scales. Nevertheless, we observe correlations by detecting photons, which travel in a clumpy universe. A proper description of galaxy number counts therefore includes relativistic terms on all scales. On small scales relativistic corrections reduce to lensing-like terms only, as shown in Eq. (2.13). Neglecting the lensing-like terms in Eq. (2.13), the leading expression simplifies to

$$
\Sigma_{\text {Newton }}=\delta^{(2)}+\mathcal{H}^{-1} \partial_{r}^{2} v^{(2)}+\left(\mathcal{H}^{-1} \partial_{r}^{2} v\right)^{2}+\mathcal{H}^{-2} \partial_{r} v \partial_{r}^{3} v+\mathcal{H}^{-1} \partial_{r} v \partial_{r} \delta+\mathcal{H}^{-1} \delta \partial_{r}^{2} v .
$$

We now show, that this expression is equivalent to the Newtonian result given in Ref. [10] (and references therein). In these references the second order perturbations are given in

Fourier space. We therefore express the real space perturbations in terms of their Fourier modes as follows

$$
\begin{gathered}
\delta^{(2)}(\mathbf{n}, z)=\frac{D_{1}^{2}}{(2 \pi)^{6}} \int d^{3} k d^{3} k_{1} d^{3} k_{2} \delta_{D}\left(\mathbf{k}-\mathbf{k}_{1}-\mathbf{k}_{2}\right) F_{2}\left(\mathbf{k}_{1}, \mathbf{k}_{2}\right) \delta\left(\mathbf{k}_{1}\right) \delta\left(\mathbf{k}_{2}\right) e^{i r(z) \mathbf{k} \cdot \mathbf{n}}, \\
\mathcal{H}^{-1} \partial_{r}^{2} v^{(2)}(\mathbf{n}, z)=\frac{D_{1}^{2} f^{2}}{(2 \pi)^{6}} \int d^{3} k d^{3} k_{1} d^{3} k_{2} \delta_{D}\left(\mathbf{k}-\mathbf{k}_{1}-\mathbf{k}_{2}\right) G_{2}\left(\mathbf{k}_{1}, \mathbf{k}_{2}\right) \delta\left(\mathbf{k}_{1}\right) \delta\left(\mathbf{k}_{2}\right) \mu^{2} e^{i r(z) \mathbf{k} \cdot \mathbf{n},} \\
\left(\mathcal{H}^{-1} \partial_{r}^{2} v(\mathbf{n}, z)\right)^{2}=\frac{D_{1}^{2} f^{2}}{(2 \pi)^{6}} \int d^{3} k_{1} d^{3} k_{2} \mu_{1}^{2} \mu_{2}^{2} \delta\left(\mathbf{k}_{1}\right) \delta\left(\mathbf{k}_{2}\right) e^{i r(z) \mathbf{k}_{1} \cdot \mathbf{n}} e^{i r(z) \mathbf{k}_{2} \cdot \mathbf{n}} \\
=\frac{D_{1}^{2} f^{2}}{(2 \pi)^{6}} \int d^{3} k d^{3} k_{1} d^{3} k_{2} \delta_{D}\left(\mathbf{k}-\mathbf{k}_{1}-\mathbf{k}_{2}\right) \mu_{1}^{2} \mu_{2}^{2} \delta\left(\mathbf{k}_{1}\right) \delta\left(\mathbf{k}_{2}\right) e^{i r(z) \mathbf{k} \cdot \mathbf{n}}, \\
\mathcal{H}^{-2} \partial_{r} v(\mathbf{n}, z) \partial_{r}^{3} v(\mathbf{n}, z) \\
=\frac{D_{1}^{2} f^{2}}{2(2 \pi)^{2}} \int d^{3} k_{1} d^{3} k_{2} \frac{\mu_{1} \mu_{2}}{k_{1} k_{2}}\left(\mu_{1}^{2} k_{1}^{2}+\mu_{2}^{2} k_{2}^{2}\right) \delta\left(\mathbf{k}_{1}\right) \delta\left(\mathbf{k}_{2}\right) e^{i r(z) \mathbf{k}_{1} \cdot \mathbf{n}} e^{i r(z) \mathbf{k}_{2} \cdot \mathbf{n}} \\
=\frac{D_{1}^{2} f^{2}}{2(2 \pi)^{6}} \int d^{3} k d^{3} k_{1} d^{3} k_{2} \delta_{D}\left(\mathbf{k}-\mathbf{k}_{1}-\mathbf{k}_{2}\right) \frac{\mu_{1} \mu_{2}}{k_{1} k_{2}}\left(\mu_{1}^{2} k_{1}^{2}+\mu_{2}^{2} k_{2}^{2}\right) \delta\left(\mathbf{k}_{1}\right) \delta\left(\mathbf{k}_{2}\right) e^{i r(z) \mathbf{k} \cdot \mathbf{n}}, \\
\mathcal{H}^{-1} \partial_{r} v \partial_{r} \delta=\frac{D_{1}^{2} f}{2(2 \pi)^{2}} \int d^{3} k_{1} d^{3} k_{2} \mu_{1} \mu_{2}\left(\frac{k_{1}}{k_{2}}+\frac{k_{2}}{k_{1}}\right) \delta\left(\mathbf{k}_{1}\right) \delta\left(\mathbf{k}_{2}\right) e^{i r(z) \mathbf{k}_{1} \cdot \mathbf{n}} e^{i r(z) \mathbf{k}_{2} \cdot \mathbf{n}} \\
=\frac{D_{1}^{2} f}{2(2 \pi)^{6}} \int d^{3} k d^{3} k_{1} d^{3} k_{2} \delta_{D}\left(\mathbf{k}-\mathbf{k}_{1}-\mathbf{k}_{2}\right) \mu_{1} \mu_{2}\left(\frac{k_{1}}{k_{2}}+\frac{k_{2}}{k_{1}}\right) \delta\left(\mathbf{k}_{1}\right) \delta\left(\mathbf{k}_{2}\right) e^{i r(z) \mathbf{k} \cdot \mathbf{n}},
\end{gathered}
$$




$$
\begin{aligned}
\mathcal{H}^{-1} \delta \partial_{r}^{2} v & =\frac{D_{1}^{2} f}{2(2 \pi)^{2}} \int d^{3} k_{1} d^{3} k_{2}\left(\mu_{1}^{2}+\mu_{2}^{2}\right) e^{i r(z) \mathbf{k}_{1} \cdot \mathbf{n}} \delta\left(\mathbf{k}_{1}\right) \delta\left(\mathbf{k}_{2}\right) e^{i r(z) \mathbf{k}_{2} \cdot \mathbf{n}} \\
& =\frac{D_{1}^{2} f}{2(2 \pi)^{6}} \int d^{3} k d^{3} k_{1} d^{3} k_{2} \delta_{D}\left(\mathbf{k}-\mathbf{k}_{1}-\mathbf{k}_{2}\right)\left(\mu_{1}^{2}+\mu_{2}^{2}\right) \delta\left(\mathbf{k}_{1}\right) \delta\left(\mathbf{k}_{2}\right) e^{i r(z) \mathbf{k} \cdot \mathbf{n}},
\end{aligned}
$$

where we have set $\mu \equiv-\hat{\mathbf{k}} \cdot \mathbf{n}, \mu_{i} \equiv-\hat{\mathbf{k}}_{i} \cdot \mathbf{n}$ and $v$ is given by the continuity equation to first order [10], $v=-\mathcal{H} k^{-2} f D_{1} \delta$. Here $\delta(\mathbf{k})$ is the initial density fluctuation and $\delta(\eta, \mathbf{k})=$ $D_{1}(\eta) \delta(\mathbf{k})$. We consider the matter dominated regime where the growth factor is independent of $k$. Combining all the terms together we find

$$
\Sigma_{\text {Newton }}(\mathbf{n}, z)=\frac{D_{1}^{2}}{(2 \pi)^{6}} \int d^{3} k d^{3} k_{1} d^{3} k_{2} \delta_{D}\left(\mathbf{k}-\mathbf{k}_{1}-\mathbf{k}_{2}\right) Z_{2}\left(\mathbf{k}_{1}, \mathbf{k}_{2}\right) \delta\left(\mathbf{k}_{1}\right) \delta\left(\mathbf{k}_{2}\right) e^{i r(z) \mathbf{k} \cdot \mathbf{n}}
$$

with

$$
Z_{2}\left(\mathbf{k}_{1}, \mathbf{k}_{2}\right)=F_{2}\left(\mathbf{k}_{1}, \mathbf{k}_{2}\right)+f^{2} \mu^{2} G_{2}\left(\mathbf{k}_{1}, \mathbf{k}_{2}\right)+\frac{f \mu k}{2}\left[\frac{\mu_{1}}{k_{1}}\left(1+f \mu_{2}^{2}\right)+\frac{\mu_{2}}{k_{2}}\left(1+f \mu_{1}^{2}\right)\right] .
$$

This result agrees with the well known Newtonian analysis, see Eq. (612) in [10]. As mentioned above, we neglect the galaxy bias factor in this work. A detailed discussion of bias can be found in [10] where also a factor $b_{2} / 2$ is included for second order bias. In the relativistic context, care has to be given to the fact that the density in comoving synchronous gauge has to be multiplied with a bias factor, see [32], but we do not address this issue here. Note also that the difference of the density perturbation in different gauges is subleading in our counting.

In addition to galaxy bias, in a realistic survey we have to consider magnification bias which takes into account that a survey usually has a limited sensitivity. Hence if a faint galaxy is lensed by the foreground mass distribution it can make it into a survey even if its apparent luminosity in the unperturbed Universe would be below the sensitivity limit of the catalog. How to include magnification bias to first order is discussed in detail in $[14,33]$. The inclusion of magnification bias to second order was recently discussed in $[22,34]$. Since it is not central to the present work, we address this problem within our framework in Appendix B, where we also point out the following interesting feature: whereas at first order the lensing term vanishes if the magnification bias is $s=2 / 5$, at second order there are always some lensing terms which do not disappear. Furthermore, new quantities enter the expressions like the perturbation of the magnification bias,

$$
-\frac{5}{2}(\delta s)^{(1)}(z, \bar{L})=\left(1+\delta^{(1)}\right) \frac{\partial \ln \rho}{\partial \ln L}-\frac{\partial \ln \bar{\rho}}{\partial \ln L},
$$

and its derivative

$$
\frac{\partial}{\partial \ln L}\left(\frac{\partial \ln \bar{\rho}}{\partial \ln L}\right)=-\frac{5}{2} t(z, \bar{L}) .
$$

Here $L$ is the luminosity of the source. If the luminosity dependence of the galaxy density, $\rho(L)$, scales as a power law, $\rho \propto L^{p}$, we have $s=-(2 / 5) p$ while $(\delta s)^{(1)}=s \delta^{(1)}$ and $t=0$.

In Appendix $\mathrm{C}$ we also derive the expressions for our dominant second order terms for the case of intensity mapping, which can be mathematically obtained simply by setting $\rho \propto L^{-1}$, so that $s=2 / 5,(\delta s)^{(1)}=2 / 5 \delta^{(1)}$ and $t=0$. As mentioned, this corresponds to 
the configuration for which the first order lensing term disappears, while at second order the transverse deflection $\left(\nabla^{a} \psi\right)\left(\nabla_{a} \delta+\mathcal{H}^{-1} \nabla_{a} \partial_{r}^{2} v\right)$ survives (see Eq. (C.3)).

In this paper, we study individually the dominant terms in the bispectrum which for a given survey have to be multiplied by the appropriate bias and magnification bias.

\section{The dominant terms in the bispectrum}

\subsection{The bispectrum}

The bispectrum of the number counts $\Delta(\mathbf{n}, z)$ is defined as the spectrum of the connected part of the expectation value

$$
B\left(\mathbf{n}_{1}, \mathbf{n}_{2}, \mathbf{n}_{3}, z_{1}, z_{2}, z_{3}\right) \equiv\left\langle\Delta\left(\mathbf{n}_{1}, z_{1}\right) \Delta\left(\mathbf{n}_{2}, z_{2}\right) \Delta\left(\mathbf{n}_{3}, z_{3}\right)\right\rangle_{c} .
$$

We can expand $\Delta$ in spherical harmonics,

$$
\Delta(\mathbf{n}, z)=\sum_{\ell=1}^{\infty} \sum_{m=-\ell}^{\ell} a_{\ell m}(z) Y_{\ell m}(\mathbf{n}), \quad a_{\ell m}(z)=\int d \Omega_{\mathbf{n}} \Delta(\mathbf{n}, z) Y_{\ell m}^{*}(\mathbf{n})
$$

and analogously

$$
B\left(\mathbf{n}_{1}, \mathbf{n}_{2}, \mathbf{n}_{3}, z_{1}, z_{2}, z_{3}\right)=\sum_{\substack{\ell_{1}, \ell_{2}, \ell_{3} \\ m_{1}, m_{2}, m_{3}}} B_{\ell_{1} \ell_{2} \ell_{3}}^{m_{1} m_{2} m_{3}}\left(z_{1}, z_{2}, z_{3}\right) Y_{\ell_{1} m_{1}}\left(\mathbf{n}_{1}\right) Y_{\ell_{2} m_{2}}\left(\mathbf{n}_{2}\right) Y_{\ell_{3} m_{3}}\left(\mathbf{n}_{3}\right),
$$

where

$$
\begin{aligned}
& B_{\ell_{1} \ell_{2} \ell_{3}}^{m_{1} m_{2} m_{3}}\left(z_{1}, z_{2}, z_{3}\right)=\left\langle a_{\ell_{1} m_{1}}\left(z_{1}\right) a_{\ell_{2} m_{2}}\left(z_{2}\right) a_{\ell_{3} m_{3}}\left(z_{3}\right)\right\rangle \\
& \quad=\int d \Omega_{1} d \Omega_{2} d \Omega_{3} B\left(\mathbf{n}_{1}, \mathbf{n}_{2}, \mathbf{n}_{3}, z_{1}, z_{2}, z_{3}\right) Y_{\ell_{1} m_{1}}^{*}\left(\mathbf{n}_{1}\right) Y_{\ell_{2} m_{2}}^{*}\left(\mathbf{n}_{2}\right) Y_{\ell_{3} m_{3}}^{*}\left(\mathbf{n}_{3}\right) .
\end{aligned}
$$

Statistical isotropy dictates the $m_{i}$ dependence of the bispectrum,

$$
B_{\ell_{1} \ell_{2} \ell_{3}}^{m_{1} m_{2} m_{3}}\left(z_{1}, z_{2}, z_{3}\right)=\mathcal{G}_{\ell_{1}, \ell_{2}, \ell_{3}}^{m_{1}, m_{2}, m_{3}} b_{\ell_{1}, \ell_{2}, \ell_{3}}\left(z_{1}, z_{2}, z_{3}\right)
$$

where $\mathcal{G}_{\ell_{1}, \ell_{2}, \ell_{3}}^{m_{1}, m_{2}, m_{3}}$ is the Gaunt integral given by

$$
\begin{aligned}
\mathcal{G}_{\ell_{1}, \ell_{2}, \ell_{3}}^{m_{1}, m_{2}, m_{3}} & =\int d \Omega Y_{\ell_{1} m_{1}}(\mathbf{n}) Y_{\ell_{2} m_{2}}(\mathbf{n}) Y_{\ell_{3} m_{3}}(\mathbf{n}) \\
& =\left(\begin{array}{ccc}
\ell_{1} & \ell_{2} & \ell_{3} \\
0 & 0 & 0
\end{array}\right)\left(\begin{array}{ccc}
\ell_{1} & \ell_{2} & \ell_{3} \\
m_{1} & m_{2} & m_{3}
\end{array}\right) \sqrt{\frac{\left(2 \ell_{1}+1\right)\left(2 \ell_{2}+1\right)\left(2 \ell_{3}+1\right)}{4 \pi}} .
\end{aligned}
$$

On the second line we have expressed the Gaunt integral in terms of the Wigner $3 j$ symbols, see e.g. [35]. The Gaunt integral is non-vanishing only for $m_{1}+m_{2}+m_{3}=0$ and $\left|\ell_{2}-\ell_{3}\right| \leq$ $\ell_{1} \leq \ell_{2}+\ell_{3}$. Furthermore, the sum $\ell_{1}+\ell_{2}+\ell_{3}$ has to be even.

We assume Gaussian initial condition so that $\left\langle\Delta^{(1)}\left(\mathbf{n}_{1}, z_{1}\right) \Delta^{(1)}\left(\mathbf{n}_{2}, z_{2}\right) \Delta^{(1)}\left(\mathbf{n}_{3}, z_{3}\right)\right\rangle_{c}=0$. We want to compute the contributions to $b_{\ell_{1}, \ell_{2}, \ell_{3}}\left(z_{1}, z_{2}, z_{3}\right)$ coming from one factor $\Delta^{(2)}$ and two factors $\Delta^{(1)}$, taking into account only the leading terms $\Sigma^{(1,2)}$, i.e.

$$
\left\langle\Delta^{(2)}\left(\mathbf{n}_{1}, z_{1}\right) \Delta^{(1)}\left(\mathbf{n}_{2}, z_{2}\right) \Delta^{(1)}\left(\mathbf{n}_{3}, z_{3}\right)\right\rangle_{c}+\text { permutations }
$$


In terms of standard perturbation theory, (3.7) is the tree-level bispectrum. Since the density fluctuation is typically the largest term in $\Delta^{(1)}$ we approximate $\Delta^{(1)} \sim \delta^{(1)}$ and we compute this part of the reduced bispectrum, which is given by

$$
\begin{aligned}
& b_{\ell_{1} \ell_{2} \ell_{3}}=b_{\ell_{1} \ell_{2} \ell_{3}}^{\delta 0}+b_{\ell_{1} \ell_{2} \ell_{3}}^{\delta 1}+b_{\ell_{1} \ell_{2} \ell_{3}}^{\delta 2}+b_{\ell_{1} \ell_{2} \ell_{3}}^{v^{(2)}}+b_{\ell_{1} \ell_{2} \ell_{3}}^{v^{\prime 2}}+b_{\ell_{1} \ell_{2} \ell_{3}}^{v v^{\prime \prime}}+b_{\ell_{1} \ell_{2} \ell_{3}}^{v \delta^{\prime}}+b_{\ell_{1} \ell_{2} \ell_{3}}^{v^{\prime} \delta} \\
& +b_{\ell_{1} \ell_{2} \ell_{3}}^{\kappa^{(2)}}+b_{\ell_{1} \ell_{2} \ell_{3}}^{\kappa \delta}+b_{\ell_{1} \ell_{2} \ell_{3}}^{\nabla \delta \nabla}+b_{\ell_{1} \ell_{2} \ell_{3}}^{v^{\prime} \kappa}+b_{\ell_{1} \ell_{2} \ell_{3}}^{\nabla v^{\prime} \nabla \psi}+b_{\ell_{1} \ell_{2} \ell_{3}}^{\kappa^{2}}+b_{\ell_{1} \ell_{2} \ell_{3}}^{\nabla \kappa \nabla \psi}+b_{\ell_{1} \ell_{2} \ell_{3}}^{\int \nabla \nabla \Psi_{1}}+b_{\ell_{1} \ell_{2} \ell_{3}}^{\int \Delta_{2}\left(\nabla \Psi_{1} \nabla \Psi_{1}\right)} .
\end{aligned}
$$

We have dropped the redshift dependence $\left(z_{1}, z_{2}, z_{3}\right)$ for sake of simplicity and we denote by $b_{\ell_{1} \ell_{2} \ell_{3}}^{\delta j}$ the monopole $(j=0)$, dipole $\left(j=1\right.$, term containing $\left.P_{1}\left(\hat{\mathbf{k}}_{1} \cdot \hat{\mathbf{k}}_{2}\right)\right)$ and quadrupole $\left(j=2\right.$, term containing $\left.P_{2}\left(\hat{\mathbf{k}}_{1} \cdot \hat{\mathbf{k}}_{2}\right)\right)$ of the bispectrum of the second order density term. We use $b_{\ell_{1} \ell_{2} \ell_{3}}^{v^{(2)^{\prime}}}$ for the second order redshift space distortion term. As for the density we shall later decompose this term into $b_{\ell_{1} \ell_{2} \ell_{3}}^{v^{\prime} j}$ with $j=0$ for the monopole, $j=1$ for the dipole and $j=2$ for the quadrupole term. The same decomposition is applied to the other pure second order term $b_{\ell_{1} \ell_{2} \ell_{3}}^{\kappa^{(2)}}$ for the second order lensing from $\Phi_{W}^{(2)}$. Again, we denote the monopole $(j=0)$, dipole $(j=1)$ and quadrupole $(j=2)$ terms by $b_{\ell_{1} \ell_{2} \ell_{3}}^{\kappa j}$. The superscripts of the other terms are according to their 2 nd order contribution:

$$
\begin{aligned}
& \text { the term containing }\left(\partial_{r}^{2} v\right)^{2} \text { is denoted by } b_{\ell_{1} \ell_{2} \ell_{3}}^{v^{2}} \text {, } \\
& \text { the term containing } \partial_{r} v \partial_{r}^{3} v \text { is denoted by } b_{\ell_{1} \ell_{2} \ell_{3}}^{v v^{\prime \prime}} \text {, } \\
& \text { the term containing } \partial_{r} v \partial_{r} \delta \text { is denoted by } b_{\ell_{1} \ell_{2} \ell_{3}}^{v \delta^{\prime}} \text {, } \\
& \text { the term containing } \partial_{r}^{2} v \delta \text { is denoted by } b_{\ell_{1} \ell_{2} \ell_{3}}^{v^{\prime}} \text {, } \\
& \text { the term containing } \kappa \delta \text { is denoted by } b_{\ell_{1} \ell_{2} \ell_{3}}^{\kappa \delta} \text {, } \\
& \text { the term containing } \nabla_{a} \delta \nabla^{a} \psi \text { is denoted by } b_{\ell_{1} \ell_{2} \ell_{3}}^{\nabla \delta \nabla \psi} \text {, } \\
& \text { the term containing } \partial_{r}^{2} v \kappa \text { is denoted by } b_{\ell_{1} \ell_{2} \ell_{3}}^{v^{\prime} \kappa} \text {, } \\
& \text { the term containing } \nabla_{a} \partial_{r}^{2} v \nabla^{a} \psi \text { is denoted by } b_{\ell_{1} \ell_{2} \ell_{3}}^{\nabla v^{\prime} \nabla \psi} \text {, } \\
& \text { the term containing } \kappa^{2} \text { is denoted by } b_{\ell_{1} \ell_{2} \ell_{3}}^{\kappa^{2}} \text {, } \\
& \text { the term containing } \nabla_{a} \kappa \nabla^{a} \psi \text { is denoted by } b_{\ell_{1} \ell_{2} \ell_{3}}^{\nabla \kappa \nabla \psi} \text {, } \\
& \text { the term containing } \int_{0}^{r(z)} \frac{d r}{r} \nabla_{a} \kappa \nabla^{a} \Psi_{1} \text { is denoted by } b_{\ell_{1} \ell_{2} \ell_{3}}^{\int \nabla \kappa} \Psi_{1} \text {, }
\end{aligned}
$$

the term containing $\int_{0}^{r(z)} d r \frac{(r(z)-r)}{r(z) r} \Delta_{2}\left(\nabla_{a} \Psi_{1} \nabla^{a} \Psi_{1}\right)$ is denoted by $b_{\ell_{1} \ell_{2} \ell_{3}}^{\int \Delta_{2}\left(\nabla \Psi_{1} \nabla \Psi_{1}\right)}$.

Eq. (3.8) is the main result of this paper. In the remainder of this section and in Appendix A we show in detail how each of its terms is calculated. In the Section 4 we present numerical results and discuss the importance of the different terms in different configurations.

The terms $b_{\ell_{1} \ell_{2} \ell_{3}}^{\delta v^{\prime}}$ and $b_{\ell_{1} \ell_{2} \ell_{3}}^{\kappa \delta}$ have already been computed in Ref. [15]. We shall not repeat their calculation here.

The first line of Eq. (3.8) refers to Newtonian terms, while the second line consists of lensing-like terms, which are not considered in the standard Newtonian analysis in Fourier space, and whose amplitude we want to quantify in this work. 


\subsection{The Newtonian terms}

To explain the basics, we give here the full derivation of the monopole part of the densitybispectrum $\left\langle\delta^{(2)}\left(\mathbf{n}_{1}, z_{1}\right) \delta^{(1)}\left(\mathbf{n}_{2}, z_{2}\right) \delta^{(1)}\left(\mathbf{n}_{3}, z_{3}\right)\right\rangle_{c}$. The computation of all the other terms is more involved and is deferred to Section 3.4 and Appendix A. In Eqs. (2.22) and (2.25) we have seen that $\delta^{(2)}$ can be written as the integral of a monopole, a dipole and a quadrupole term. Let us first just consider the monopole term which we call $\delta^{(2) 0}$. We introduce the initial curvature power spectrum from linear perturbation theory by

$$
\left\langle R_{\text {in }}(\mathbf{k}) R_{\text {in }}\left(\mathbf{k}^{\prime}\right)\right\rangle=(2 \pi)^{3} \delta_{D}\left(\mathbf{k}+\mathbf{k}^{\prime}\right) P_{R}(k) .
$$

For a given variable $A$ we define the transfer function $T_{A}(\eta, k)$ by

$$
A(\eta, \mathbf{k})=T_{A}(\eta, k) R_{\text {in }}(\mathbf{k}) .
$$

This determines $A$ within linear perturbation theory. We will also use the angular power spectra given by

$$
c_{\ell}^{A B}\left(z_{1}, z_{2}\right)=4 \pi \int \frac{d k}{k} \mathcal{P}_{R}(k) \Delta_{\ell}^{A}\left(z_{1}, k\right) \Delta_{\ell}^{B}\left(z_{2}, k\right)=\frac{2}{\pi} \int d k k^{2} P_{R}(k) \Delta_{\ell}^{A}\left(z_{1}, k\right) \Delta_{\ell}^{B}\left(z_{2}, k\right),
$$

where $\mathcal{P}_{R}(k)=\frac{k^{3}}{2 \pi^{2}} P_{R}(k)$ is the dimensionless primordial power spectrum. $\Delta_{\ell}^{A}(z, k)$ denotes the transfer function in angular and redshift space for the variable $A$. For example, for the first order density fluctuation $\delta$ the Fourier representation

$$
\delta(\mathbf{n}, z)=\frac{1}{(2 \pi)^{3}} \int d^{3} k \delta(k, \eta(z)) e^{i r(z) \mathbf{k} \cdot \mathbf{n}}
$$

yields

$$
\Delta_{\ell}^{\delta}(z, k)=T_{\delta}(\eta(z), k) j_{\ell}(k r(z)),
$$

where $j_{\ell}(x)$ is the spherical Bessel function of order $\ell$. For the monopole term of $\delta^{(2)}$,

$$
\delta^{(2) 0}\left(\mathbf{k}_{1}, \eta\right)=\frac{17}{21} \frac{1}{(2 \pi)^{3}} \int d^{3} k \delta^{(1)}(\mathbf{k}, \eta) \delta^{(1)}\left(\mathbf{k}_{1}-\mathbf{k}, \eta\right)
$$

we then obtain

$$
\begin{aligned}
\left\langle\delta^{(2) 0}\right. & \left.\left(\mathbf{n}_{1}, z_{1}\right) \delta^{(1)}\left(\mathbf{n}_{2}, z_{2}\right) \delta^{(1)}\left(\mathbf{n}_{3}, z_{3}\right)\right\rangle_{c} \\
= & \frac{17}{21} \frac{2}{(2 \pi)^{6}} \int d^{3} k_{2} d^{3} k_{3} P\left(k_{2}\right) P\left(k_{3}\right) T_{\delta}\left(k_{2}, \eta_{1}\right) T_{\delta}\left(k_{3}, \eta_{1}\right) \\
& \times T_{\delta}\left(k_{2}, \eta_{2}\right) T_{\delta}\left(k_{3}, \eta_{3}\right) e^{-i\left(\mathbf{k}_{2} \cdot \mathbf{n}_{1} r_{1}+\mathbf{k}_{3} \cdot \mathbf{n}_{1} r_{1}\right)} e^{i\left(\mathbf{k}_{2} \cdot \mathbf{n}_{2} r_{2}+\mathbf{k}_{3} \cdot \mathbf{n}_{3} r_{3}\right)} .
\end{aligned}
$$

Expanding the Fourier modes in spherical harmonics and Bessel functions

$$
e^{i \mathbf{k} \cdot \mathbf{n} r}=4 \pi \sum_{\ell m} i^{\ell} j_{\ell}(k r) Y_{\ell m}(\mathbf{n}) Y_{\ell m}^{*}(\hat{\mathbf{k}})
$$

and integrating over the angles $\hat{\mathbf{k}}_{2}$ and $\hat{\mathbf{k}}_{3}$ applying the orthogonality of spherical harmonics, we find the three-point function

$$
\begin{aligned}
B^{\delta 0}\left(\mathbf{n}_{1}, \mathbf{n}_{2}, \mathbf{n}_{3}, z_{1}, z_{2}, z_{3}\right)= & \frac{4}{\pi^{2}} \sum_{\ell, \ell^{\prime}, m, m^{\prime}} Y_{\ell m}\left(\mathbf{n}_{1}\right) Y_{\ell^{\prime} m^{\prime}}\left(\mathbf{n}_{1}\right) Y_{\ell m}^{*}\left(\mathbf{n}_{2}\right) Y_{\ell^{\prime} m^{\prime}}^{*}\left(\mathbf{n}_{3}\right) F_{\ell \ell^{\prime}}\left(z_{1}, z_{2}, z_{3}\right) \\
& + \text { perm. }
\end{aligned}
$$


with

$$
\begin{gathered}
F_{\ell \ell^{\prime}}\left(z_{1}, z_{2}, z_{3}\right)=\frac{34}{21} \int d k_{2} d k_{3} k_{2}^{2} k_{3}^{2} P_{R}\left(k_{2}\right) P_{R}\left(k_{3}\right) T_{\delta}\left(\eta_{1}, k_{2}\right) T_{\delta}\left(\eta_{1}, k_{3}\right) T_{\delta}\left(\eta_{2}, k_{2}\right) T_{\delta}\left(\eta_{3}, k_{3}\right) \\
\times j_{\ell}\left(k_{2} r_{1}\right) j_{\ell^{\prime}}\left(k_{3} r_{1}\right) j_{\ell}\left(k_{2} r_{2}\right) j_{\ell^{\prime}}\left(k_{3} r_{3}\right) .
\end{gathered}
$$

From (3.16) we infer the reduced bispectrum defined in Eq. (3.4),

$$
b_{\ell_{1} \ell_{2} \ell_{3}}^{\delta 0}=\frac{4}{\pi^{2}} F_{\ell_{2} \ell_{3}}\left(z_{1}, z_{2}, z_{3}\right)+\text { perm. . }
$$

The double integral in (3.17) is simply a product of two single integrals so that we can simplify the result to

$$
\begin{aligned}
b_{\ell_{1} \ell_{2} \ell_{3}}^{\delta 0}= & \frac{34}{21}\left[\frac{2}{\pi} \int d k_{2} k_{2}^{2} P_{R}\left(k_{2}\right) T_{\delta}\left(\eta_{1}, k_{2}\right) T_{\delta}\left(\eta_{2}, k_{2}\right) j_{\ell_{2}}\left(k_{2} r_{1}\right) j_{\ell_{2}}\left(k_{2} r_{2}\right)\right] \\
& \times\left[\frac{2}{\pi} \int d k_{3} k_{3}^{2} P_{R}\left(k_{3}\right) T_{\delta}\left(\eta_{1}, k_{3}\right) T_{\delta}\left(\eta_{3}, k_{3}\right) j_{\ell_{3}}\left(k_{3} r_{1}\right) j_{\ell_{3}}\left(k_{3} r_{3}\right)\right]+\text { perm. } \\
= & \frac{34}{21}\left[c_{\ell_{2}}^{\delta \delta}\left(z_{1}, z_{2}\right) c_{\ell_{3}}^{\delta \delta}\left(z_{1}, z_{3}\right)+c_{\ell_{1}}^{\delta \delta}\left(z_{1}, z_{2}\right) c_{\ell_{3}}^{\delta \delta}\left(z_{2}, z_{3}\right)+c_{\ell_{1}}^{\delta \delta}\left(z_{1}, z_{3}\right) c_{\ell_{2}}^{\delta \delta}\left(z_{2}, z_{3}\right)\right]
\end{aligned}
$$

Here $c_{\ell}^{\delta \delta}\left(z_{i}, z_{j}\right)$ is the contribution to the number count angular power spectrum from density fluctuations. It can be calculated with the publicly available code CLAssgal described in [33]. The notation '+ perm.' always indicates that all different permutations have to be considered. Since the expressions are always symmetrical in the second and third index, see Eq. (3.7), this means that all three values of $\left(\ell_{i}, z_{i}\right)$ have to be considered once in the first position. As mentioned, we set the bias $b$ between matter and galaxies equal to one, but it is easy to add a linear and scale-independent bias as outlined in CLAss.

The derivation of the dipole and the quadrupole terms, as well as all the velocity and lensing contributions, are given in Appendix A. We use in particular the definitions in Eq. (A.8) for the generalized angular spectra ${ }^{n} c_{\ell \ell^{\prime}}^{A B}\left(z_{1}, z_{2}\right)$, and Eqs. (A.6) and (A.11) for the geometrical factors $g_{\ell_{1} \ell_{2} \ell_{3}}$ and $Q_{\ell^{\prime} \ell^{\prime \prime}}^{\ell_{1} \ell_{3}}$ describing the bispectra in terms of Wigner $3 j$ and $6 j$ symbols. The results for these contributions to the bispectrum are as follows:

$$
\begin{aligned}
b_{\ell_{1} \ell_{2} \ell_{3}}^{\delta 1}\left(z_{1}, z_{2}, z_{3}\right)= & \left(g_{\ell_{1} \ell_{2} \ell_{3}}\right)^{-1} \frac{1}{16 \pi^{2}} \sum_{\ell^{\prime} \ell^{\prime \prime}}\left(2 \ell^{\prime}+1\right)\left(2 \ell^{\prime \prime}+1\right) Q_{1}^{\ell_{1} \ell_{2} \ell_{3} \ell^{\prime \prime}} \\
& \times\left[{ }^{1} c_{\ell^{\prime \prime} \ell_{1}}^{\delta \delta}\left(z_{3}, z_{1}\right){ }^{-1} c_{\ell^{\prime} \ell_{2}}^{\delta \delta}\left(z_{3}, z_{2}\right)+{ }^{-1} c_{\ell^{\prime \prime} \ell_{1}}^{\delta \delta}\left(z_{3}, z_{1}\right)^{1} c_{\ell^{\prime} \ell_{2}}^{\delta \delta}\left(z_{3}, z_{2}\right)\right] \\
& + \text { perm. }
\end{aligned}
$$

which is non-vanishing only if $\ell^{\prime}=\ell_{2} \pm 1$ and $\ell^{\prime \prime}=\ell_{1} \pm 1$, and

$$
\begin{aligned}
b_{\ell_{1} \ell_{2} \ell_{3}}^{\delta 2}\left(z_{1}, z_{2}, z_{3}\right)= & \left(g_{\ell_{1} \ell_{2} \ell_{3}}\right)^{-1} \frac{1}{42 \pi^{2}} \sum_{\ell^{\prime} \ell^{\prime \prime}}\left(2 \ell^{\prime}+1\right)\left(2 \ell^{\prime \prime}+1\right) Q_{2}^{\ell_{1} \ell_{2} \ell_{3} \ell_{3}^{\prime \prime}}{ }^{0} c_{\ell^{\prime \prime} \ell_{1}}^{\delta \delta}\left(z_{3}, z_{1}\right){ }^{0} c_{\ell^{\prime} \ell_{2}}^{\delta \delta}\left(z_{3}, z_{2}\right) \\
& + \text { perm. }
\end{aligned}
$$

which is non-vanishing only if $\ell^{\prime}=\ell_{2} \pm 2, \ell_{2}$ and $\ell^{\prime \prime}=\ell_{1} \pm 2$.

Here and in what follows we denote $\eta(z)=\eta$ and $r(z)=\eta_{0}-\eta(z)=r$. Correspondingly $\eta^{\prime}=\eta\left(z^{\prime}\right)$, etc. . 
Defining the transfer functions

$$
\begin{aligned}
\Delta_{\ell}^{v}(z, k) & =T_{v}(k, \eta) j_{\ell}^{\prime}(k r), \\
\Delta_{\ell}^{v^{\prime}}(z, k) & =\frac{k}{\mathcal{H}(z)} T_{v}(k, \eta) j_{\ell}^{\prime \prime}(k r), \\
\Delta_{\ell}^{v^{\prime \prime}}(z, k) & =\frac{k^{2}}{\mathcal{H}^{2}(z)} T_{v}(k, \eta) j_{\ell}^{\prime \prime \prime}(k r),
\end{aligned}
$$

we can express the reduced bispectrum for the term $\partial_{r} v \partial_{r}^{3} v$ as (see Appendix A.2)

$$
b_{\ell_{1} \ell_{2} \ell_{3}}^{v v^{\prime \prime}}\left(z_{1}, z_{2}, z_{3}\right)=\left[c_{\ell_{2}}^{v \delta}\left(z_{1}, z_{2}\right) c_{\ell_{3}}^{v^{\prime \prime} \delta}\left(z_{1}, z_{3}\right)+c_{\ell_{2}}^{v^{\prime \prime} \delta}\left(z_{1}, z_{2}\right) c_{\ell_{3}}^{v \delta}\left(z_{1}, z_{3}\right)\right]+\text { perm. }
$$

and (see Appendix A.3 and A.4 and [15] for details)

$$
\begin{aligned}
& b_{\ell_{1} \ell_{2} \ell_{3}}^{v^{\prime 2}}\left(z_{1}, z_{2}, z_{3}\right)=2 c_{\ell_{2}}^{v^{\prime} \delta}\left(z_{1}, z_{2}\right) c_{\ell_{3}}^{v^{\prime} \delta}\left(z_{1}, z_{3}\right)+\text { perm. } \\
& b_{\ell_{1} \ell_{2} \ell_{3}}^{v \delta^{\prime}}\left(z_{1}, z_{2}, z_{3}\right)=\left[c_{\ell_{2}}^{v \delta}\left(z_{1}, z_{2}\right) c_{\ell_{3}}^{\delta^{\prime} \delta}\left(z_{1}, z_{3}\right)+c_{\ell_{2}}^{\delta^{\prime} \delta}\left(z_{1}, z_{2}\right) c_{\ell_{3}}^{v \delta}\left(z_{1}, z_{3}\right)\right]+\text { perm. } \\
& b_{\ell_{1} \ell_{2} \ell_{3}}^{v^{\prime} \delta}\left(z_{1}, z_{2}, z_{3}\right)=\left[c_{\ell_{2}}^{v^{\prime} \delta}\left(z_{1}, z_{2}\right) c_{\ell_{3}}^{\delta \delta}\left(z_{1}, z_{3}\right)+c_{\ell_{2}}^{\delta \delta}\left(z_{1}, z_{2}\right) c_{\ell_{3}}^{v^{\prime} \delta}\left(z_{1}, z_{3}\right)\right]+\text { perm. }
\end{aligned}
$$

where we have also introduced also the transfer function

$$
\Delta_{\ell}^{\delta^{\prime}}(z, k)=\frac{k}{\mathcal{H}(z)} T_{\delta}(k, \eta) j_{\ell}^{\prime}(k r) .
$$

The contributions to the bispectrum given so far can all also be found in [10] where they are given in $k$-space in Eq. (620), which is not directly related to observables. However, the conversion of these results to $k$-space is straightforward and we have checked that our results are in perfect agreement with Ref. [10]. Apart from the $v^{(2)}$ term which we calculate in Section 3.4, these are all the Newtonian terms.

\subsection{Terms containing lensing}

The contributions which we describe below are new. They are induced by lensing and, as we shall see, are relevant especially for large redshift differences or wide redshift bins. Their translation to $k$-space is not so straightforward as they are integrals over the backward lightcone.

We express the lensing-like terms by introducing the following transfer functions,

$$
\begin{aligned}
\Delta_{\ell}^{\kappa}(z, k) & =\ell(\ell+1) \int_{0}^{r(z)} d r \frac{r(z)-r}{r(z) r} T_{\Psi+\Phi}\left(\eta_{0}-r, k\right) j_{\ell}(k r), \\
\Delta_{\ell}^{\Psi_{1}}(z, k) & =\frac{1}{r(z)} \int_{0}^{r(z)} d r T_{\Psi+\Phi}\left(\eta_{0}-r, k\right) j_{\ell}(k r) .
\end{aligned}
$$

With this we find (see Appendix A.6 to A.13 for details)

$$
\begin{aligned}
& b_{\ell_{1} \ell_{2} \ell_{3}}^{\kappa \delta}\left(z_{1}, z_{2}, z_{3}\right)=\left[c_{\ell_{2}}^{\kappa \delta}\left(z_{1}, z_{2}\right) c_{\ell_{3}}^{\delta \delta}\left(z_{1}, z_{3}\right)+c_{\ell_{3}}^{\kappa \delta}\left(z_{1}, z_{3}\right) c_{\ell_{2}}^{\delta \delta}\left(z_{1}, z_{2}\right)\right]+\text { perm. } \\
& b_{\ell_{1} \ell_{2} \ell_{3}}^{v^{\prime} \kappa}\left(z_{1}, z_{2}, z_{3}\right)=\left[c_{\ell_{2}}^{v^{\prime} \delta}\left(z_{1}, z_{2}\right) c_{\ell_{3}}^{\kappa \delta}\left(z_{1}, z_{3}\right)+c_{\ell_{2}}^{\kappa \delta}\left(z_{1}, z_{2}\right) c_{\ell_{3}}^{v^{\prime} \delta}\left(z_{1}, z_{3}\right)\right]+\text { perm. } \\
& b_{\ell_{1} \ell_{2} \ell_{3}}^{\kappa^{2}}\left(z_{1}, z_{2}, z_{3}\right)=c_{\ell_{2}}^{\kappa \delta}\left(z_{1}, z_{2}\right) c_{\ell_{3}}^{\kappa \delta}\left(z_{1}, z_{3}\right)+\text { perm. }
\end{aligned}
$$




$$
\begin{aligned}
& b_{\ell_{1} \ell_{2} \ell_{3}}^{\nabla \delta \nabla \psi}\left(z_{1}, z_{2}, z_{3}\right)=A_{\ell_{1} \ell_{2} \ell_{3}}\left[\sqrt{\frac{\ell_{3}\left(\ell_{3}+1\right)}{\ell_{2}\left(\ell_{2}+1\right)}} c_{\ell_{2}}^{\kappa \delta}\left(z_{1}, z_{2}\right) c_{\ell_{3}}^{\delta \delta}\left(z_{1}, z_{3}\right)\right. \\
& \left.+\sqrt{\frac{\ell_{2}\left(\ell_{2}+1\right)}{\ell_{3}\left(\ell_{3}+1\right)}} c_{\ell_{3}}^{\kappa \delta}\left(z_{1}, z_{3}\right) c_{\ell_{2}}^{\delta \delta}\left(z_{1}, z_{2}\right)\right]+ \text { perm. }, \\
& b_{\ell_{1} \ell_{2} \ell_{3}}^{\nabla v^{\prime} \nabla \psi}\left(z_{1}, z_{2}, z_{3}\right)=A_{\ell_{1} \ell_{2} \ell_{3}}\left[\sqrt{\frac{\ell_{3}\left(\ell_{3}+1\right)}{\ell_{2}\left(\ell_{2}+1\right)}} c_{\ell_{2}}^{\kappa \delta}\left(z_{1}, z_{2}\right) c_{\ell_{3}}^{v^{\prime} \delta}\left(z_{1}, z_{3}\right)\right. \\
& \left.+\sqrt{\frac{\ell_{2}\left(\ell_{2}+1\right)}{\ell_{3}\left(\ell_{3}+1\right)}} c_{\ell_{3}}^{\kappa \delta}\left(z_{1}, z_{3}\right) c_{\ell_{2}}^{v^{\prime} \delta}\left(z_{1}, z_{2}\right)\right]+ \text { perm. } \\
& b_{\ell_{1} \ell_{2} \ell_{3}}^{\nabla \kappa \nabla \psi}\left(z_{1}, z_{2}, z_{3}\right)=A_{\ell_{1} \ell_{2} \ell_{3}} \frac{\ell_{2}\left(\ell_{2}+1\right)+\ell_{3}\left(\ell_{3}+1\right)}{\sqrt{\ell_{2}\left(\ell_{2}+1\right) \ell_{3}\left(\ell_{3}+1\right)}} c_{\ell_{2}}^{\kappa \delta}\left(z_{1}, z_{2}\right) c_{\ell_{3}}^{\kappa \delta}\left(z_{1}, z_{3}\right)+\text { perm., }(3.3 \\
& b_{\ell_{1} \ell_{2} \ell_{3}}^{\int \nabla \kappa \nabla \Psi_{1}}\left(z_{1}, z_{2}, z_{3}\right)=-A_{\ell_{1} \ell_{2} \ell_{3}} \sqrt{\ell_{2}\left(\ell_{2}+1\right)} \sqrt{\ell_{3}\left(\ell_{3}+1\right)} \\
& \times \int_{0}^{r_{1}} \frac{d r}{r}\left[c_{\ell_{2}}^{\kappa \delta}\left(z, z_{2}\right) c_{\ell_{3}}^{\Psi_{1} \delta}\left(z, z_{3}\right)+c_{\ell_{3}}^{\kappa \delta}\left(z, z_{3}\right) c_{\ell_{2}}^{\Psi_{1} \delta}\left(z, z_{2}\right)\right]+\text { perm. } \\
& b_{\ell_{1} \ell_{2} \ell_{3}}^{\int \Delta_{2}\left(\nabla \Psi_{1} \nabla \Psi_{1}\right)}\left(z_{1}, z_{2}, z_{3}\right)=-\left\{A_{\ell_{1} \ell_{2} \ell_{3}} \sqrt{\ell_{2}\left(\ell_{2}+1\right) \ell_{3}\left(\ell_{3}+1\right)}\left[\ell_{2}\left(\ell_{2}+1\right)+\ell_{3}\left(\ell_{3}+1\right)\right]\right. \\
& \left.+C_{\ell_{1} \ell_{2} \ell_{3}} \sqrt{\frac{\left(\ell_{2}+2\right) !}{\left(\ell_{2}-2\right) !}} \sqrt{\frac{\left(\ell_{3}+2\right) !}{\left(\ell_{3}-2\right) !}}+\ell_{2}\left(\ell_{2}+1\right) \ell_{3}\left(\ell_{3}+1\right)\right\} \\
& \times \int_{0}^{r_{1}} d r \frac{r_{1}-r}{r_{1} r} c_{\ell_{2}}^{\Psi_{1} \delta}\left(z, z_{2}\right) c_{\ell_{3}}^{\Psi_{1} \delta}\left(z, z_{3}\right)+\text { perm. } .
\end{aligned}
$$

Again $A_{\ell_{1} \ell_{2} \ell_{3}}$ and $C_{\ell_{1} \ell_{2} \ell_{3}}$ are combinations of generalised Gaunt factors which are defined in Appendix A, Eqs. (A.72) and (A.108). In the integrals of the last two equations $z \equiv z(r)$. The derivation of Eqs. (3.28) and (3.32) can be found in [15], all other results are derived in Appendix A.

\subsection{Bispectrum for the second order velocity and lensing terms}

All the contributions to the bispectrum computed so far can be written in terms of products of two point functions. But this is not the case for the second order velocity, $v^{(2)}$, and the second order lensing, $\kappa^{(2)}$ terms. Because of the pre-factors $k^{-2}=\left(\mathbf{k}_{1}+\mathbf{k}_{2}\right)^{-2}$ in Eqs. (2.23) and (2.24), which can not be written as products of functions of $\mathbf{k}_{1}$ and $\mathbf{k}_{2}$.

Here we derive analytical expression for these second order terms. They turn out to be numerically much more involved as they require not two single integrals but a full quadruple integral. For the $\kappa^{(2)}$ term the additional integration over the background light-cone even leads to a quintuple integral.

However, since we are interested mainly in orders of magnitudes, we shall simplify these expressions by using the Limber approximation [36, 37]. This is equivalent to the flat sky approximation and should be reasonable for $\ell_{i}>30$ or so (of course we have in principle 3 different flat skies as we consider 3 different redshifts). We shall also repeat the derivation of the bispectrum from $\delta^{(2)}$ with the Limber approximation. This gives us an indication of the precision of this approach. The Limber approximation has been efficiently used for second order weak lensing calculations [38] and our treatment is inspired by Ref. [38]. 


\subsubsection{Second order redshift space distortion}

Let us first compute the bispectrum for the redshift space distortion at second order. We consider

$$
\left\langle\left(\mathcal{H}^{-1} \partial_{r_{1}}^{2} v^{(2)}\right)\left(\mathbf{n}_{1}, z_{1}\right) \delta^{(1)}\left(\mathbf{n}_{2}, z_{2}\right) \delta^{(1)}\left(\mathbf{n}_{3}, z_{3}\right)\right\rangle_{c} \equiv\langle\cdots\rangle
$$

where $v^{(2)}$ is given in Eqs. (2.23) and (2.26). We find

$$
\begin{aligned}
\langle\cdots\rangle & =-\frac{f^{2}\left(z_{1}\right)}{(2 \pi)^{12}} \int d^{3} k d^{3} k^{\prime} d^{3} k_{1} d^{3} k_{2} d^{3} k_{3} \delta_{D}\left(\mathbf{k}_{1}-\mathbf{k}-\mathbf{k}^{\prime}\right) \frac{G_{2}\left(\mathbf{k}, \mathbf{k}^{\prime}\right)}{k_{1}^{2}}\left(\partial_{r_{1}}^{2} e^{i \mathbf{k}_{1} \cdot \mathbf{n}_{1} r_{1}}\right) \\
& \times e^{i\left(\mathbf{k}_{2} \cdot \mathbf{n}_{2} r_{2}+\mathbf{k}_{3} \cdot \mathbf{n}_{3} r_{3}\right)} T_{\delta}\left(k, \eta_{1}\right) T_{\delta}\left(k^{\prime}, \eta_{1}\right) T_{\delta}\left(k_{2}, \eta_{2}\right) T_{\delta}\left(k_{3}, \eta_{3}\right)\left\langle R(\mathbf{k}) R\left(\mathbf{k}^{\prime}\right) R\left(\mathbf{k}_{2}\right) R\left(\mathbf{k}_{3}\right)\right\rangle \\
= & -\frac{2 f^{2}\left(z_{1}\right)}{(2 \pi)^{6}} \int d^{3} k_{1} d^{3} k_{2} d^{3} k_{3} \delta_{D}\left(\mathbf{k}_{1}+\mathbf{k}_{2}+\mathbf{k}_{3}\right) G_{2}\left(-\mathbf{k}_{2},-\mathbf{k}_{3}\right)\left(\partial_{\left(k_{1} r_{1}\right)}^{2} e^{i \mathbf{k}_{1} \cdot \mathbf{n}_{1} r_{1}}\right) \\
& \times e^{i\left(\mathbf{k}_{2} \cdot \mathbf{n}_{2} r_{2}+\mathbf{k}_{3} \cdot \mathbf{n}_{3} r_{3}\right)} T_{\delta}\left(k_{2}, \eta_{1}\right) T_{\delta}\left(k_{3}, \eta_{1}\right) T_{\delta}\left(k_{2}, \eta_{2}\right) T_{\delta}\left(k_{3}, \eta_{3}\right) P\left(k_{2}\right) P\left(k_{3}\right) \\
= & -\frac{2 f^{2}\left(z_{1}\right)}{\pi^{3}} \sum_{\ell_{i}, m_{i}} \int d^{3} k_{1} d^{3} k_{2} d^{3} k_{3} \delta_{D}\left(\mathbf{k}_{1}+\mathbf{k}_{2}+\mathbf{k}_{3}\right) G_{2}\left(\mathbf{k}_{2}, \mathbf{k}_{3}\right) P\left(k_{2}\right) P\left(k_{3}\right) \\
& \times i^{\ell_{1}+\ell_{2}+\ell_{3}} j_{\ell_{1}}^{\prime \prime}\left(k_{1} r_{1}\right) j_{\ell_{2}}\left(k_{2} r_{2}\right) j_{\ell_{3}}\left(k_{3} r_{3}\right) Y_{\ell_{1} m_{1}}^{*}\left(\hat{\mathbf{k}}_{1}\right) Y_{\ell_{2} m_{2}}^{*}\left(\hat{\mathbf{k}}_{2}\right) Y_{\ell_{3} m_{3}}^{*}\left(\hat{\mathbf{k}}_{3}\right) \\
& \times Y_{\ell_{1} m_{1}}\left(\mathbf{n}_{1}\right) Y_{\ell_{2} m_{2}}\left(\mathbf{n}_{2}\right) Y_{\ell_{3} m_{3}}\left(\mathbf{n}_{3}\right) T_{\delta}\left(k_{2}, \eta_{1}\right) T_{\delta}\left(k_{3}, \eta_{1}\right) T_{\delta}\left(k_{2}, \eta_{2}\right) T_{\delta}\left(k_{3}, \eta_{3}\right) .
\end{aligned}
$$

Note that here, when using the Dirac-delta to eliminate $\mathbf{k}_{1}$, we obtain $j_{\ell_{1}}^{\prime \prime}\left(\left|\mathbf{k}_{2}+\mathbf{k}_{3}\right| r_{1}\right)$ which cannot be written as a product of functions of $\mathbf{k}_{2}$ and $\mathbf{k}_{3}$ as we always had it so far. To continue we rewrite the Dirac-delta distribution as

$$
\begin{aligned}
\delta_{D}\left(\mathbf{k}_{1}+\mathbf{k}_{2}+\mathbf{k}_{3}\right)= & \frac{1}{(2 \pi)^{3}} \int d^{3} x e^{i\left(\mathbf{k}_{1}+\mathbf{k}_{2}+\mathbf{k}_{3}\right) \mathbf{x}} \\
= & 8 \sum_{\ell_{i}^{\prime}, m_{i}^{\prime}} i^{\ell_{1}^{\prime}+\ell_{2}^{\prime}+\ell_{3}^{\prime}}(-1)^{\ell_{1}^{\prime}+\ell_{2}^{\prime}+\ell_{3}^{\prime}} \mathcal{G}_{\ell_{1}^{\prime}, \ell_{2}^{\prime}, \ell_{3}^{\prime}}^{m^{\prime}, m_{3}^{\prime}, m_{\ell_{1}^{\prime}}^{\prime}} Y_{\ell_{1}^{\prime}}\left(\hat{\mathbf{k}}_{1}\right) Y_{\ell_{2}^{\prime} m_{2}^{\prime}}\left(\hat{\mathbf{k}}_{2}\right) Y_{\ell_{3}^{\prime} m_{3}^{\prime}}\left(\hat{\mathbf{k}}_{3}\right) \\
& \times \int_{0}^{\infty} d \chi \chi^{2} j_{\ell_{1}^{\prime}}\left(k_{1} \chi\right) j_{\ell_{2}^{\prime}}\left(k_{2} \chi\right) j_{\ell_{3}^{\prime}}\left(k_{3} \chi\right),
\end{aligned}
$$

and the kernel $G_{2}$ as

$$
G_{2}\left(k_{1}, k_{2}, k_{3}\right) \equiv G_{2}\left(\mathbf{k}_{2}, \mathbf{k}_{3}\right)=\frac{3}{7}+\frac{1}{4} \frac{k_{1}^{2}-k_{2}^{2}-k_{3}^{2}}{k_{2} k_{3}}\left(\frac{k_{2}}{k_{3}}+\frac{k_{3}}{k_{2}}\right)+\frac{1}{7}\left(\frac{k_{1}^{2}-k_{2}^{2}-k_{3}^{2}}{k_{2} k_{3}}\right)^{2} .
$$

When inserting the third side of our triangle in $k$-space, $k_{1}=\left|\mathbf{k}_{2}+\mathbf{k}_{3}\right|$, the angular dependence of $G_{2}$ disappears (or rather is hidden in $k_{1}$ ). With this we find

$$
\begin{aligned}
\langle\cdots\rangle= & -\frac{16 f^{2}\left(z_{1}\right)}{\pi^{3}} \sum_{\ell_{i}, m_{i}} \mathcal{G}_{\ell_{1}, \ell_{2}, \ell_{3}}^{m_{1}, m_{2}, m_{3}} \int d k_{1} d k_{2} d k_{3} k_{1}^{2} k_{2}^{2} k_{3}^{2} G_{2}\left(k_{1}, k_{2}, k_{3}\right) P\left(k_{2}\right) P\left(k_{3}\right) \\
& \times j_{\ell_{1}}^{\prime \prime}\left(k_{1} r_{1}\right) j_{\ell_{2}}\left(k_{2} r_{2}\right) j_{\ell_{3}}\left(k_{3} r_{3}\right) T_{\delta}\left(k_{2}, \eta_{1}\right) T_{\delta}\left(k_{3}, \eta_{1}\right) T_{\delta}\left(k_{2}, \eta_{2}\right) T_{\delta}\left(k_{3}, \eta_{3}\right) \\
& \times \int_{0}^{\infty} d \chi \chi^{2} j_{\ell_{1}}\left(k_{1} \chi\right) j_{\ell_{2}}\left(k_{2} \chi\right) j_{\ell_{3}}\left(k_{3} \chi\right) Y_{\ell_{1} m_{1}}\left(\mathbf{n}_{1}\right) Y_{\ell_{2} m_{2}}\left(\mathbf{n}_{2}\right) Y_{\ell_{3} m_{3}}\left(\mathbf{n}_{3}\right) .
\end{aligned}
$$


With this we obtain for the bispectrum defined in (3.2)

$$
\begin{aligned}
B_{\ell_{1} \ell_{2} \ell_{3}}^{m_{1} m_{2} m_{3}}\left(z_{1}, z_{2}, z_{3}\right) & =-\frac{16 f^{2}\left(z_{1}\right)}{\pi^{3}} \mathcal{G}_{\ell_{1}, \ell_{2}, \ell_{3}}^{m_{1}, m_{2}, m_{3}} \int d k_{1} d k_{2} d k_{3} k_{1}^{2} k_{2}^{2} k_{3}^{2} G_{2}\left(k_{1}, k_{2}, k_{3}\right) P\left(k_{2}\right) P\left(k_{3}\right) \\
& \times j_{\ell_{1}}^{\prime \prime}\left(k_{1} r_{1}\right) j_{\ell_{2}}\left(k_{2} r_{2}\right) j_{\ell_{3}}\left(k_{3} r_{3}\right) T_{\delta}\left(k_{2}, \eta_{1}\right) T_{\delta}\left(k_{3}, \eta_{1}\right) T_{\delta}\left(k_{2}, \eta_{2}\right) T_{\delta}\left(k_{3}, \eta_{3}\right) \\
& \times \int_{0}^{\infty} d \chi \chi^{2} j_{\ell_{1}}\left(k_{1} \chi\right) j_{\ell_{2}}\left(k_{2} \chi\right) j_{\ell_{3}}\left(k_{3} \chi\right)+\text { perm. },
\end{aligned}
$$

and the reduced bispectrum becomes

$$
\begin{aligned}
b_{\ell_{1} \ell_{2} \ell_{3}}^{v^{(2)^{\prime}}}\left(z_{1}, z_{2}, z_{3}\right)= & -\frac{16 f^{2}\left(z_{1}\right)}{\pi^{3}} \int d k_{1} d k_{2} d k_{3} k_{1}^{2} k_{2}^{2} k_{3}^{2} G_{2}\left(k_{1}, k_{2}, k_{3}\right) P\left(k_{2}\right) P\left(k_{3}\right) \\
& \times j_{\ell_{1}}^{\prime \prime}\left(k_{1} r_{1}\right) j_{\ell_{2}}\left(k_{2} r_{2}\right) j_{\ell_{3}}\left(k_{3} r_{3}\right) T_{\delta}\left(k_{2}, \eta_{1}\right) T_{\delta}\left(k_{3}, \eta_{1}\right) T_{\delta}\left(k_{2}, \eta_{2}\right) T_{\delta}\left(k_{3}, \eta_{3}\right) \\
& \times \int_{0}^{\infty} d \chi \chi^{2} j_{\ell_{1}}\left(k_{1} \chi\right) j_{\ell_{2}}\left(k_{2} \chi\right) j_{\ell_{3}}\left(k_{3} \chi\right)+\text { perm.. }
\end{aligned}
$$

In this expression it is important to first evaluate the integral over $\chi$. If we would naively change the order of integration and first integrate over $k_{j}$, the integral would diverge. However, when integrating over $\chi$ in the situation where, for example, $k_{1}>k_{2}+k_{3}$ there exists an analytic solution of the $\chi$-integral (see e.g. [39], integral no. 1 of 6.578 ) which can be written in the form

$$
\int_{0}^{\infty} d \chi \chi^{2} j_{\ell_{1}}\left(k_{1} \chi\right) j_{\ell_{2}}\left(k_{2} \chi\right) j_{\ell_{3}}\left(k_{3} \chi\right) \propto \frac{\alpha\left(\frac{k_{2}}{k_{1}}, \frac{k_{3}}{k_{1}} ; \ell_{1}, \ell_{2}, \ell_{3}\right)}{\Gamma\left(-\frac{\ell_{3}+\ell_{2}-\ell_{1}}{2}\right)},
$$

where the numerator $\alpha$ is finite. Furthermore, we need to consider only the combinations of $\left(\ell_{1}, \ell_{2}, \ell_{3}\right)$ for which the Gaunt factor $\mathcal{G}_{\ell_{1}, \ell_{2}, \ell_{3}}^{m_{1}, m_{3}}$ does not vanish, namely $\ell_{1}+\ell_{2}+\ell_{3}$ has to be a even integer and $\left|\ell_{3}-\ell_{2}\right| \leq \ell_{1} \leq \ell_{3}+\ell_{2}$. But this implies that the argument of the Gamma function is an non-positive integer. Because the Gamma function has poles at non-positive integer arguments, the integral (3.47) vanishes when the triangle inequality for the $k_{i}$ is violated. We therefore can impose the condition $k_{1}<k_{2}+k_{3}$ on the integral over $k_{1}$. Similarly, we can also impose the conditions $k_{2}<k_{1}+k_{3}$ and $k_{3}<k_{1}+k_{2}$. Imposing these bounds for the integrations over $k_{i}$, the integral converges unconditionally and we are allowed to change the order of integration at will. We then define

$$
\begin{aligned}
\mathcal{I}\left(k_{2}, k_{3}, \chi, r_{1}, \ell_{1}\right)= & \int d k_{1} k_{1}^{2} \Theta\left(k_{2}+k_{3}-k_{1}\right) \Theta\left(k_{1}+k_{3}-k_{2}\right) \Theta\left(k_{1}+k_{2}-k_{3}\right) \\
& \times G_{2}\left(k_{1}, k_{2}, k_{3}\right) j_{\ell_{1}}^{\prime \prime}\left(k_{1} r_{1}\right) j_{\ell_{1}}\left(k_{1} \chi\right)
\end{aligned}
$$

where the $\Theta$ denotes the Heaviside step function, and re-write the reduced bispectrum (3.46) as

$$
\begin{aligned}
& b_{\ell_{1} \ell_{2} \ell_{3}}^{v^{(2)^{\prime}}}\left(z_{1}, z_{2}, z_{3}\right)=-\frac{16 f^{2}\left(z_{1}\right)}{\pi^{3}} \int d \chi d k_{2} d k_{3} \chi^{2} k_{2}^{2} k_{3}^{2} P_{R}\left(k_{2}\right) P_{R}\left(k_{3}\right) j_{\ell_{2}}\left(k_{2} r_{2}\right) j_{\ell_{3}}\left(k_{3} r_{3}\right) \\
& \times j_{\ell_{2}}\left(k_{2} \chi\right) j_{\ell_{3}}\left(k_{3} \chi\right) T_{\delta}\left(k_{2}, \eta_{1}\right) T_{\delta}\left(k_{3}, \eta_{1}\right) T_{\delta}\left(k_{2}, \eta_{2}\right) T_{\delta}\left(k_{3}, \eta_{3}\right) \mathcal{I}\left(k_{2}, k_{3}, \chi, r_{1}, \ell_{1}\right) \\
& \text { +perm. . }
\end{aligned}
$$

This is a nested quadruple integral which would have to be evaluated numerically with Monte Carlo techniques. At the present time this goes beyond the capacities of our numerical code 
and to obtain a first order of magnitude result we use the Limber approximation for the integrals over $k_{2}$ and $k_{3}$. This means we approximate

$$
\frac{2}{\pi} \int d k k^{2} f(k) j_{\ell}\left(k x_{1}\right) j_{\ell}\left(k x_{2}\right) \simeq \frac{\delta_{D}\left(x_{1}-x_{2}\right)}{x_{1}^{2}} f\left(\frac{\ell+1 / 2}{x_{1}}\right) .
$$

Applying Eq. (3.50) twice we obtain

$$
\begin{aligned}
b_{\ell_{1} \ell_{2} \ell_{3}}^{v^{(2)^{\prime}}}\left(z_{1}, z_{2}, z_{3}\right) \simeq & -\frac{4 f^{2}\left(z_{1}\right)}{\pi} \frac{\delta_{D}\left(r_{2}-r_{3}\right)}{r_{2}^{2}} P_{R}\left(\nu_{2}\right) P_{R}\left(\nu_{3}\right) T_{\delta}\left(\nu_{2}, \eta_{1}\right) T_{\delta}\left(\nu_{3}, \eta_{1}\right) \\
& \times T_{\delta}\left(\nu_{2}, \eta_{2}\right) T_{\delta}\left(\nu_{3}, \eta_{3}\right) \mathcal{I}\left(\nu_{2}, \nu_{3}, r_{3}, r_{1}, \ell_{1}\right)+\text { perm. }
\end{aligned}
$$

where $\nu_{i} \equiv \frac{\ell_{i}+1 / 2}{r_{i}}$. Hence the 2 nd order RSD bispectrum of Eq. (3.51) has a 'contact term'. It is non-vanishing only if the redshifts $z_{2}$ and $z_{3}$ are equal. Also $\mathcal{I}\left(\nu_{2}, \nu_{3}, r_{3}, r_{1}, \ell_{1}\right)$ is rapidly oscillating if $r_{3} \neq r_{1}$ so this is close to another Dirac-delta of $r_{3}-r_{1}$. This is certainly an approximation, but it reflects the fact that the RSD bispectrum rapidly decreases with increasing redshift difference. Note also that we have to convolve $b^{v^{(2)^{\prime}}}$ with a window function in redshift space to obtain a finite result in this approximation.

We could try to use the Limber approximation also to evaluate Eq. (3.48). We start from the identity

$$
j_{\ell_{1}}^{\prime \prime}\left(k_{1} r_{1}\right)=\frac{1}{\left(k_{1} r_{1}\right)^{2}}\left\{\left[\ell_{1}\left(\ell_{1}-1\right)-\left(k_{1} r_{1}\right)^{2}\right] j_{\ell_{1}}\left(k_{1} r_{1}\right)+2 k_{1} r_{1} j_{\ell_{1}+1}\left(k_{1} r_{1}\right)\right\}
$$

and, following [40] we approximate $j_{\ell_{1}+1}(x) \simeq j_{\ell_{1}}(x)$. Since the Limber approximation is valid for $\ell_{1} \gg 1$ and it implies $\ell_{1} \sim k_{1} r_{1}$, applying the Limber approximation of Eq. (3.50) to Eq. (3.48) the leading terms in $\ell_{1}$ cancel. This is a strong indication that the contribution (3.51) of the second order RSD to the total bispectrum is actually subleading with respect to the second order density contribution, which is given below in Eq. (3.60) in the Limber approximation.

\subsubsection{Second order lensing}

We now compute the bispectrum for the lensing to second order. We start from

$$
\left\langle-2 \kappa^{(2)}\left(\mathbf{n}_{1}, z_{1}\right) \delta^{(1)}\left(\mathbf{n}_{2}, z_{2}\right) \delta^{(1)}\left(\mathbf{n}_{3}, z_{3}\right)\right\rangle_{c}
$$

where $\kappa^{(2)}$ is given in Eq. (2.14). Following the same procedure used above for the RSD at second order, we obtain the following expression

$$
\begin{aligned}
& b_{\ell_{1} \ell_{2} \ell_{3}}^{\kappa^{(2)}}\left(z_{1}, z_{2}, z_{3}\right)=-\frac{48}{\pi^{3}} \int_{0}^{r_{1}} d r \frac{r_{1}-r}{r_{1} r} \mathcal{H}^{2}(\eta) \Omega_{m}(\eta) \int d \chi d k_{2} d k_{3} \chi^{2} k_{2}^{2} k_{3}^{2} P_{R}\left(k_{2}\right) P_{R}\left(k_{3}\right) \\
& \quad \times j_{\ell_{2}}\left(k_{2} \chi\right) j_{\ell_{3}}\left(k_{3} \chi\right) j_{\ell_{2}}\left(k_{2} r_{2}\right) j_{\ell_{3}}\left(k_{3} r_{3}\right) T_{\delta}\left(k_{2}, \eta^{\prime}\right) T_{\delta}\left(k_{3}, \eta^{\prime}\right) T_{\delta}\left(k_{2}, \eta_{2}\right) T_{\delta}\left(k_{3}, \eta_{3}\right) \\
& \quad \times \mathcal{J}\left(k_{2}, k_{3}, \chi, r, \ell_{1}\right)+\text { perm. }
\end{aligned}
$$

where the two additional permutations are, as usual, on the redshift and multipole pairs $\left\{z_{i}, \ell_{i}\right\}$, and

$$
\begin{aligned}
\mathcal{J}\left(k_{2}, k_{3}, \chi, r, \ell_{1}\right)= & \ell_{1}\left(\ell_{1}+1\right) \int d k_{1} \Theta\left(k_{2}+k_{3}-k_{1}\right) \Theta\left(k_{1}+k_{3}-k_{2}\right) \Theta\left(k_{1}+k_{2}-k_{3}\right) \\
& \times F_{2}\left(k_{1}, k_{2}, k_{3}\right) j_{\ell_{1}}\left(k_{1} r\right) j_{\ell_{1}}\left(k_{1} \chi\right),
\end{aligned}
$$


with

$$
F_{2}\left(k_{1}, k_{2}, k_{3}\right) \equiv F_{2}\left(\mathbf{k}_{2}, \mathbf{k}_{3}\right)=\frac{5}{7}+\frac{1}{4} \frac{k_{1}^{2}-k_{2}^{2}-k_{3}^{2}}{k_{2} k_{3}}\left(\frac{k_{2}}{k_{3}}+\frac{k_{3}}{k_{2}}\right)+\frac{1}{14}\left(\frac{k_{1}^{2}-k_{2}^{2}-k_{3}^{2}}{k_{2} k_{3}}\right)^{2} .
$$

Finally, the Limber approximation for the $k_{1}, k_{2}$ and $k_{3}$ integrations in Eqs. (3.54) and (3.55), leads to

$$
\begin{aligned}
b_{\ell_{1} \ell_{2} \ell_{3}}^{\kappa^{(2)}}\left(z_{1}, z_{2}, z_{3}\right)= & -24 \Theta\left(z_{1}-z_{3}\right) \frac{r_{1}-r_{3}}{r_{1} r_{3}} \mathcal{H}^{2}\left(\eta_{3}\right) \Omega_{m}\left(\eta_{3}\right) \frac{\delta_{D}\left(r_{2}-r_{3}\right)}{r_{3}^{2}} \\
& \times P_{R}\left(\nu_{2}\right) P_{R}\left(\nu_{3}\right) T_{\delta}^{2}\left(\nu_{2}, \eta_{3}\right) T_{\delta}^{2}\left(\nu_{3}, \eta_{3}\right) \frac{\ell_{1}\left(\ell_{1}+1\right)}{\left(2 \ell_{1}+1\right)^{2}} F_{2}\left(\frac{\ell_{1}+1 / 2}{r_{3}}, \nu_{2}, \nu_{3}\right) \\
& + \text { perm. }
\end{aligned}
$$

The three Heaviside step functions of Eq. (3.55) reduce to $\ell_{i}+\ell_{j}-\ell_{n}>0$ which is trivially satisfied for all terms with non-vanishing Gaunt factor.

\subsubsection{Comparison with the second order density}

We have already evaluated the second order density bispectrum exactly. In Appendix A we obtain the three terms (3.19), (3.20) and (3.21). Here we re-compute the bispectrum for the density to second order using the Limber approximation in order to evaluate the difference.

Following the same procedure as above we find

$$
\begin{aligned}
& b_{\ell_{1} \ell_{2} \ell_{3}}^{\delta^{(2)}}\left(z_{1}, z_{2}, z_{3}\right)=\frac{16}{\pi^{3}} \int d \chi d k_{2} d k_{3} \chi^{2} k_{2}^{2} k_{3}^{2} P_{R}\left(k_{2}\right) P_{R}\left(k_{3}\right) j_{\ell_{2}}\left(k_{2} \chi\right) j_{\ell_{3}}\left(k_{3} \chi\right) \\
& \quad \times j_{\ell_{2}}\left(k_{2} r_{2}\right) j_{\ell_{3}}\left(k_{3} r_{3}\right) T_{\delta}\left(k_{2}, \eta_{1}\right) T_{\delta}\left(k_{3}, \eta_{1}\right) T_{\delta}\left(k_{2}, \eta_{2}\right) T_{\delta}\left(k_{3}, \eta_{3}\right) \mathcal{K}\left(k_{2}, k_{3}, \chi, r_{1}, \ell_{1}\right) \\
& \quad+\text { perm. }
\end{aligned}
$$

where

$$
\begin{aligned}
\mathcal{K}\left(k_{2}, k_{3}, \chi, r_{1}, \ell_{1}\right)= & \int d k_{1} k_{1}^{2} \Theta\left(k_{2}+k_{3}-k_{1}\right) \Theta\left(k_{1}+k_{3}-k_{2}\right) \Theta\left(k_{1}+k_{2}-k_{3}\right) \\
& \times F_{2}\left(k_{1}, k_{2}, k_{3}\right) j_{\ell_{1}}\left(k_{1} r_{1}\right) j_{\ell_{1}}\left(k_{1} \chi\right) .
\end{aligned}
$$

Applying the Limber approximation, Eq. (3.50), we then obtain

$$
\begin{aligned}
b_{\ell_{1} \ell_{2} \ell_{3}}^{\delta^{(2)}}\left(z_{1}, z_{2}, z_{3}\right)= & 2 \frac{\delta_{D}\left(r_{2}-r_{3}\right) \delta_{D}\left(r_{1}-r_{3}\right)}{r_{3}^{4}} P_{R}\left(\nu_{2}\right) P_{R}\left(\nu_{3}\right) T_{\delta}^{2}\left(\nu_{2}, \eta_{3}\right) \\
& \times T_{\delta}^{2}\left(\nu_{3}, \eta_{3}\right) F_{2}\left(\frac{\ell_{1}+1 / 2}{r_{3}}, \nu_{2}, \nu_{3}\right)+\text { perm. }
\end{aligned}
$$

The result in Eq. (3.60) is useful for two reasons. First, comparing it with the exact bispectrum obtained in Eqs. (3.19), (3.20) and (3.21), we can test the accuracy of the Limber approximation when applied to the calculation of the three-point function. Secondly, comparing Eqs. (3.57) and (3.60), integrated over window functions tells us for which situations the lensing contribution to second order is comparable to the density contribution to second order. From the above expressions it is clear that the 2nd order lensing bispectrum is significant when two redshifts are equal and the third is larger, while the 2 nd order density 
(or RSD) bispectrum only contributes when all redshifts are (nearly) equal. Interestingly $b_{\ell_{1} \ell_{2} \ell_{3}}^{\kappa^{(2)}}\left(z_{1}, z_{2}, z_{3}\right)$ can be obtained from $b_{\ell_{1} \ell_{2} \ell_{3}}^{\delta^{(2)}}\left(z_{1}, z_{2}, z_{3}\right)$ upon replacing

$$
\delta_{D}\left(r_{1}-r_{3}\right) \rightarrow-12\left(r_{1}-r_{3}\right) \frac{r_{3}}{r_{1}} \Theta\left(r_{1}-r_{3}\right) \frac{\ell_{1}\left(\ell_{1}+1\right)}{\left(2 \ell_{1}+1\right)^{2}} \mathcal{H}^{2}\left(z_{3}\right) \Omega_{m}\left(z_{3}\right) .
$$

For window functions of radial size $\mathcal{H}^{-1}$, we therefore expect that the two contributions are of the same order.

The results in Eqs. (3.51), (3.57) and (3.60) can be split into their monopole, dipole and quadrupole contribution (comparing Eqs. (3.56) and (3.43) with Eqs. (2.25) and (2.26)) in the following way

$$
\begin{gathered}
b_{\ell_{1} \ell_{2} \ell_{3}}^{v^{\prime} j}\left(z_{1}, z_{2}, z_{3}\right)=-\frac{4 f^{2}\left(z_{1}\right)}{\pi} \frac{\delta_{D}\left(r_{2}-r_{3}\right)}{r_{3}^{2}} P_{R}\left(\nu_{2}\right) P_{R}\left(\nu_{3}\right) T_{\delta}\left(\nu_{2}, \eta_{1}\right) T_{\delta}\left(\nu_{3}, \eta_{1}\right) T_{\delta}\left(\nu_{2}, \eta_{2}\right) \\
\times T_{\delta}\left(\nu_{3}, \eta_{3}\right) \mathcal{I}_{j}\left(\nu_{2}, \nu_{3}, r_{3}, r_{1}, \ell_{1}\right)+\text { perm. }
\end{gathered}
$$

with

$$
\begin{aligned}
\mathcal{I}_{0}\left(k_{2}, k_{3}, r_{3}, r_{1}, \ell_{1}\right)= & \int d k_{1} k_{1}^{2} \Theta\left(k_{2}+k_{3}-k_{1}\right) \Theta\left(k_{1}+k_{3}-k_{2}\right) \Theta\left(k_{1}+k_{2}-k_{3}\right) \\
& \times \frac{13}{21} j_{\ell_{1}}^{\prime \prime}\left(k_{1} r_{1}\right) j_{\ell_{1}}\left(k_{1} r_{3}\right), \\
\mathcal{I}_{1}\left(k_{2}, k_{3}, r_{3}, r_{1}, \ell_{1}\right)= & \int d k_{1} k_{1}^{2} \Theta\left(k_{2}+k_{3}-k_{1}\right) \Theta\left(k_{1}+k_{3}-k_{2}\right) \Theta\left(k_{1}+k_{2}-k_{3}\right) \\
& \times \frac{1}{4} \frac{k_{1}^{2}-k_{2}^{2}-k_{3}^{2}}{k_{2} k_{3}}\left(\frac{k_{2}}{k_{3}}+\frac{k_{3}}{k_{2}}\right) j_{\ell_{1}}^{\prime \prime}\left(k_{1} r_{1}\right) j_{\ell_{1}}\left(k_{1} r_{3}\right), \\
\mathcal{I}_{2}\left(k_{2}, k_{3}, r_{3}, r_{1}, \ell_{1}\right)= & \int d k_{1} k_{1}^{2} \Theta\left(k_{2}+k_{3}-k_{1}\right) \Theta\left(k_{1}+k_{3}-k_{2}\right) \Theta\left(k_{1}+k_{2}-k_{3}\right) \\
& \times\left[\frac{1}{7}\left(\frac{k_{1}^{2}-k_{2}^{2}-k_{3}^{2}}{k_{2} k_{3}}\right)^{2}-\frac{4}{21}\right] j_{\ell_{1}}^{\prime \prime}\left(k_{1} r_{1}\right) j_{\ell_{1}}\left(k_{1} r_{3}\right) .
\end{aligned}
$$

While for lensing and density we have

$$
\begin{aligned}
b_{\ell_{1} \ell_{2} \ell_{3}}^{\kappa j}\left(z_{1}, z_{2}, z_{3}\right)= & -24 \Theta\left(z_{1}-z_{3}\right) \frac{r_{1}-r_{3}}{r_{1} r_{3}} \mathcal{H}^{2}\left(\eta_{3}\right) \Omega_{m} \frac{\delta_{D}\left(r_{2}-r_{3}\right)}{r_{3}^{2}} P_{R}\left(\nu_{2}\right) P_{R}\left(\nu_{3}\right) \\
& \times T_{\delta}^{2}\left(\nu_{2}, \eta_{3}\right) T_{\delta}^{2}\left(\nu_{3}, \eta_{3}\right) \frac{\ell_{1}\left(\ell_{1}+1\right)}{\left(2 \ell_{1}+1\right)^{2}} F_{2}^{(j)}\left(\frac{\ell_{1}+1 / 2}{r_{3}}, \nu_{2}, \nu_{3}\right)+\text { perm. }, \\
b_{\ell_{1} \ell_{2} \ell_{3}}^{\delta j}\left(z_{1}, z_{2}, z_{3}\right)= & 2 \frac{\delta_{D}\left(r_{2}-r_{3}\right) \delta_{D}\left(r_{1}-r_{3}\right)}{r_{3}^{4}} P_{R}\left(\nu_{2}\right) P_{R}\left(\nu_{3}\right) \\
& \times T_{\delta}^{2}\left(\nu_{2}, \eta_{1}\right) T_{\delta}^{2}\left(\nu_{3}, \eta_{1}\right) F_{2}^{(j)}\left(\frac{\ell_{1}+1 / 2}{r_{3}}, \nu_{2}, \nu_{3}\right)+\text { perm. }
\end{aligned}
$$


with

$$
\begin{aligned}
& F_{2}^{(0)}\left(k_{1}, k_{2}, k_{3}\right)=\frac{17}{21}, \\
& F_{2}^{(1)}\left(k_{1}, k_{2}, k_{3}\right)=\frac{1}{4} \frac{k_{1}^{2}-k_{2}^{2}-k_{3}^{2}}{k_{2} k_{3}}\left(\frac{k_{2}}{k_{3}}+\frac{k_{3}}{k_{2}}\right), \\
& F_{2}^{(2)}\left(k_{1}, k_{2}, k_{3}\right)=\left[\frac{1}{14}\left(\frac{k_{1}^{2}-k_{2}^{2}-k_{3}^{2}}{k_{2} k_{3}}\right)^{2}-\frac{2}{21}\right] .
\end{aligned}
$$

Due to the two Dirac- $\delta$ 's, the second order density term (3.66) is non-vanishing only if all three redshifts coincide. For the lensing term instead is sufficient if two redshifts coincide while the third one is larger than these two. Clearly in this configuration the two density fluctuations in the foreground lens the background density. In Appendix A we also give the Limber approximation for the $\kappa^{2}$-term, see Eq. (A.58). In this case there are two line of sight integrals leading to two Heaviside- $\Theta$ functions and the signal remains substantial also when all three redshifts are different. Of course, the Limber approximation is not exact and the signal for the second order density term does not disappear if the redshifts are different, but as we shall see in the next section, for the Newtonian (non-lensing) contributions the signal decays rapidly with increasing redshift difference. Even though for the velocity term (3.61) there is only one Dirac- $\delta$, the integrals $\mathcal{I}_{j}$ in practice act like an additional $\delta\left(r_{3}-r_{1}\right)$.

In the next section we evaluate numerically the reduced bispectrum associated to the different contributions given in Sec. 3.1 and 3.4. For this purpose we will divide our terms in three different types: Newtonian terms, Newtonian $\times$ lensing terms and pure lensing contribution. The Newtonian terms are the one given by the first line of Eq. (3.8), the Newtonian $\times$ lensing ones are combinations of lensing terms with Newtonian ones and are given by the first five terms of the second line of Eq. (3.8), including the $\kappa^{(2)}$-term, and the pure lensing terms are the last four of the second line of Eq. (3.8).

Let us, in particular, explain why we consider $\kappa^{(2)}$ as a Newtonian $\times$ lensing term. From a naive point of view, one might be tempted to consider $\kappa^{(2)}$ as a pure second order lensing term. On the other hand, considering its form and Eq. (3.57) it is evident that this term resembles more to a combination of a lensing term with the density. To clarify this let us apply the Limber approximation to the bispectrum of the $\kappa \delta$ term. Starting from the result of [15], where the reduced bispectrum of this term was given in detail. Using the Poisson equation (2.24) and applying the Limber approximation we obtain

$$
\begin{aligned}
b_{\ell_{1} \ell_{2} \ell_{3}}^{\kappa \delta}\left(z_{1}, z_{2}, z_{3}\right)=-12 \Theta & \left(z_{1}-z_{3}\right) \frac{r_{1}-r_{3}}{r_{1} r_{3}} \mathcal{H}^{2}\left(\eta_{3}\right) \Omega_{m}\left(\eta_{3}\right) \frac{\delta_{D}\left(r_{1}-r_{2}\right)}{r_{2}^{2}} \\
& \times P_{R}\left(\nu_{2}\right) P_{R}\left(\nu_{3}\right) T_{\delta}^{2}\left(\nu_{2}, \eta_{2}\right) T_{\delta}^{2}\left(\nu_{3}, \eta_{3}\right) \frac{\ell_{3}\left(\ell_{3}+1\right)}{\left(2 \ell_{3}+1\right)^{2}} \\
- & 12 \Theta\left(z_{1}-z_{2}\right) \frac{r_{1}-r_{2}}{r_{1} r_{2}} \mathcal{H}^{2}\left(\eta_{2}\right) \Omega_{m}\left(\eta_{2}\right) \frac{\delta_{D}\left(r_{1}-r_{3}\right)}{r_{3}^{2}} \\
& \times P_{R}\left(\nu_{2}\right) P_{R}\left(\nu_{3}\right) T_{\delta}^{2}\left(\nu_{2}, \eta_{2}\right) T_{\delta}^{2}\left(\nu_{3}, \eta_{3}\right) \frac{\ell_{2}\left(\ell_{2}+1\right)}{\left(2 \ell_{2}+1\right)^{2}} \\
+ & \text { perm. }
\end{aligned}
$$

Comparing Eq. (3.70) to Eq. (3.57), clearly the bispectrum from $\kappa \delta$ and $\kappa^{(2)}$ are very similar (apart from the kernel $F_{2}$, which is not present in (3.70)). The difference is simply that 
the $\delta$ term has fixed redshift for $\kappa \delta$, while it is inside the integral along the line of sight for $\kappa^{(2)}$. As we shall see in the next section, Eq. (3.57) produces similar results as the other Newtonian $\times$ lensing terms when we consider our bispectrum integrated over window functions (see Sec. 4.1). As a consequence, we consider $\kappa^{(2)}$ as a Newtonian $\times$ lensing term, and not as a pure lensing terms. This also indicates that this second order lensing effect plays a role also when two redshifts are equal, a configuration where the other pure lensing terms are negligible as we shall see.

\section{Numerical examples and how to measure lensing terms}

In this section we show some numerical examples of our results. The figures have been generated with the cosmological parameters $h=0.67, \omega_{b}=0.022, \omega_{c d m}=0.12$, and vanishing curvature. The primordial curvature power spectrum has the amplitude $A_{s}=2.215 \times 10^{-9}$, the pivot scale $k_{\text {pivot }}=0.05 \mathrm{Mpc}^{-1}$, the spectral index $n_{s}=0.96$ and no running. All the transfer functions needed to compute the spectra are evaluated with a modified version of the CLASS code [33, 41].

In Figs. 1 and 2 we show the contributions to the bispectrum from $\delta^{(2)}$ which are expected to dominate the result. We compare the exact result with the Limber approximation and find that even though the order of magnitude agrees, the results from the Limber approximation can differ by nearly a factor of 2 . At closer inspection we have found that the Limber approximation for the monopole is excellent, whereas the global difference is due to the relatively slow convergence of the integrands associated to the dipole and quadrupole terms in the UV tail of our region of integration. Nevertheless, for a first indication of the amplitude we use the Limber approximation for $v^{(2)}$ and $\kappa^{(2)}$ for comparison with the new lensing terms. For $\delta^{(2)}$ we just consider the monopole in the following figures.

In Fig. 1 one sees how the curvature of $Q=1+\left(b^{\delta 1}+b^{\delta 2}\right) / b^{\delta 0}$ increases with decreasing redshift, i.e. when gravitational structure grows and enhances the asymmetry of the 3-point function. These shapes and their evolution with redshift are excellent tests of (Newtonian) gravity. They are due to the fact that gravity tends to 'flatten' triangular configurations. For equal redshifts this means that one opening angle at the observer position should be roughly equal to the sum or the difference of the other two to maximize the bispectrum. We have also investigated different configurations in $\ell$-space and the accuracy of the Limber approximation remains similar. The order of magnitude is correct but the result can be up to $40 \%$ off also for large $\ell$.

In Fig. 2 we show the density monopole (Eq. (3.19), first term of Eq. (3.8), first panel), the dipole (Eq. (3.20), second term of Eq. (3.8), second panel) and the quadrupole (Eq. (3.21), third term of Eq. (3.8), third panel). The negative dipole term $b^{\delta 1}$ is mainly responsible for the shape of Fig. 1 .

In Fig. 3 we show the curvature of the second order velocity term using Limber approximation, $P=1+\left(b^{v^{\prime} 1}+b^{v^{\prime} 2}\right) / b^{v^{\prime} 0}$. As explained in section 3.4.1 also the second order RSD (forth term in Eq. (3.8)) can be split into a monopole dipole and quadrupole term denoted by $b^{v^{\prime} 0}, b^{v^{\prime} 1}$ and $b^{v^{\prime} 2}$ which are given (within the Limber approximation) in Eq. (3.61). While the Limber approximation should give roughly the right shape of the curvature $P$ (like for the second order density case, compare top and bottom panels of Fig. 1), the relative amplitude of the dipole and quadrupole terms with respect to the monopole term may be quite inaccurate both in magnitude and in sign (similarly to the case of the second order density bispectrum). In the bispectrum from the second order velocity term, the dipole has again 

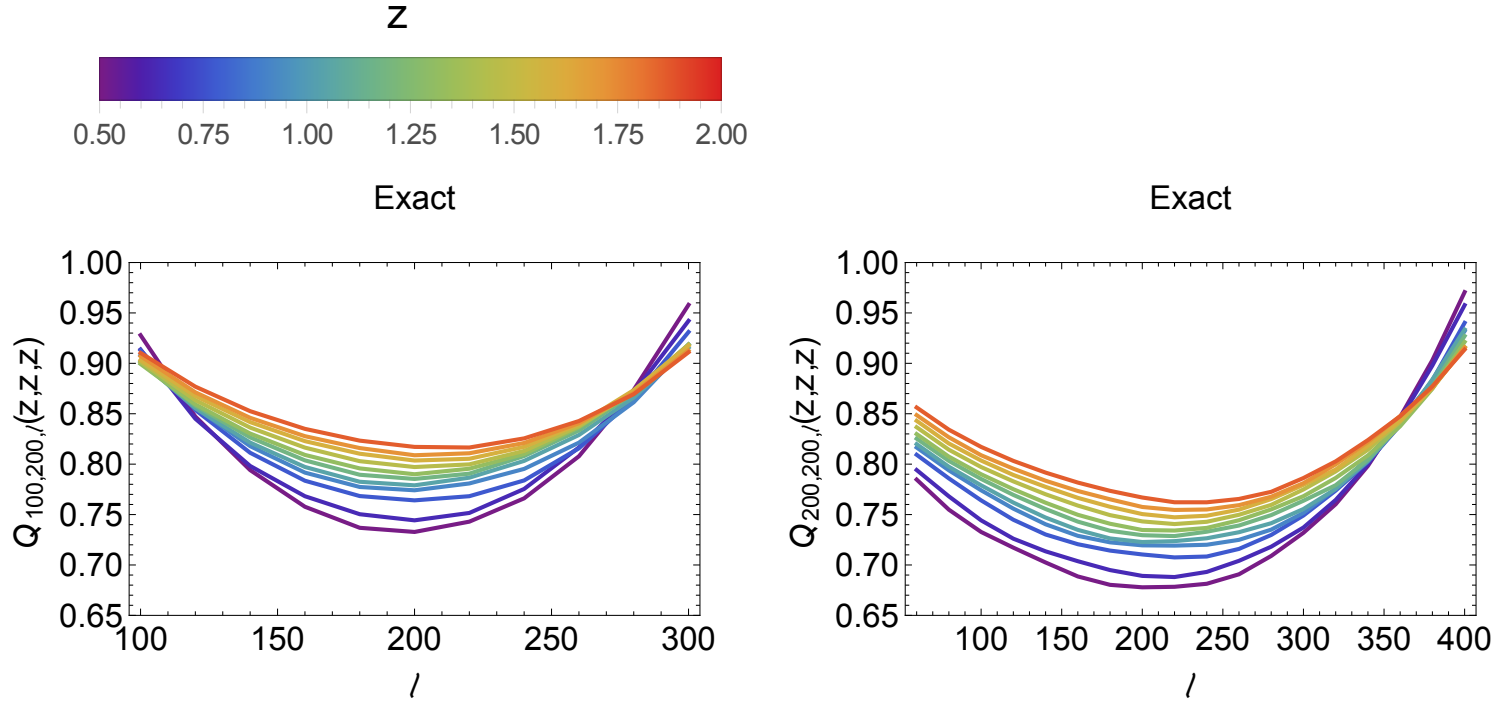

Limber
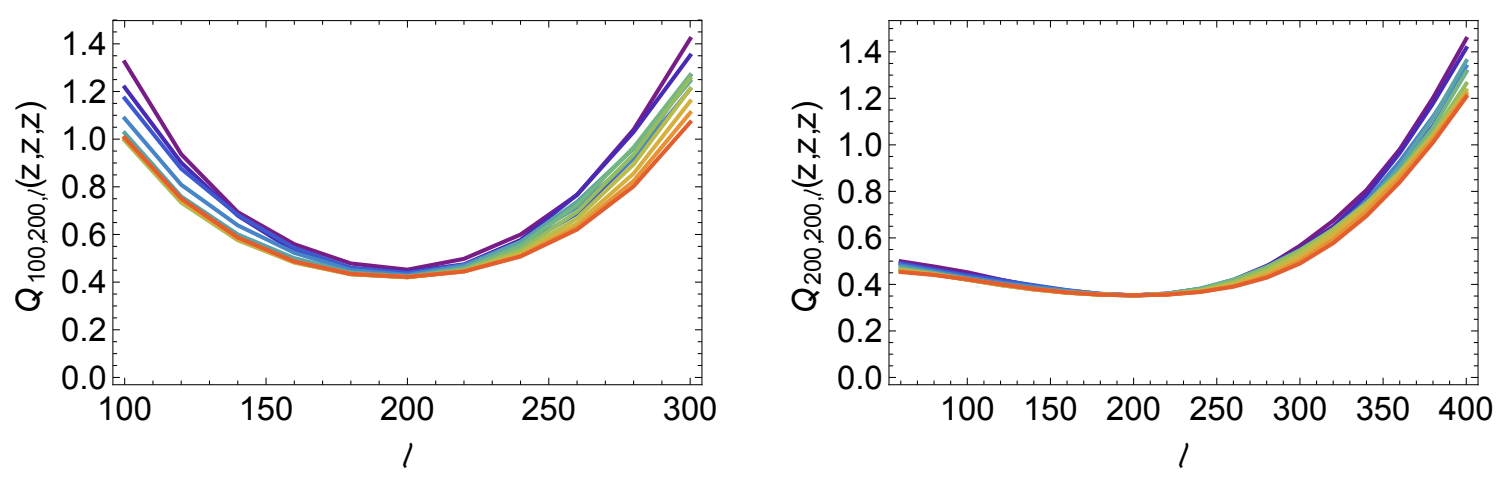

Figure 1. We plot $Q_{\ell_{1} \ell_{2} \ell_{3}}\left(z_{1}, z_{2}, z_{3}\right) \equiv 1+\left(b_{\ell_{1} \ell_{2} \ell_{3}}^{\delta 1}+b_{\ell_{1} \ell_{2} \ell_{3}}^{\delta 2}\right) / b_{\ell_{1} \ell_{2} \ell_{3}}^{\delta 0}$. The upper panels show the exact computation, and for comparison in the lower panels the Limber approximation has been applied. Different lines denote different redshift from $z=0.5$ (purple) to $z=2$ (red). The deviation from the monopole increases at low redshift, because non-Gaussianities generated by non-linear evolution grow in time. Whereas the analogous quantity plotted in Fourier space is by construction redshiftindependent. The Limber approximation (lower panel) gives the right order of magnitude and roughly the correct shape of the ratio $Q$ as a function of $\ell$ but does not agree by about $30 \%$ to $40 \%$ also at high $\ell \gtrsim 100$.

the opposite sign but the quadrupole is significantly larger and increases the bispectrum especially for $1 / \ell_{2}+1 / \ell_{3}=1 / \ell_{1}$.

In Fig. 4 we show the contributions from the Newtonian terms of $\Sigma^{(2)}$. These are the 1st and 5th to 8th terms in Eq. (3.8). Their explicite expressions are given in Eqs. (3.19) for the 1st panel, (3.26) for the 2nd panel, (3.25) for the 3rd panel, (3.27) for the 4th panel and (3.28) for the 5 th panel. We do not plot $b_{\ell_{1} \ell_{2} \ell_{3} \ell^{\prime}}$, which we can only determine within the Limber approximation, see Eq. (3.51), where it has a contact term (Dirac-delta) so that it can not be shown in the configuration of Fig. 4, where no window function is included. We fix the redshifts $z_{1}=z_{2}=1$ and plot the terms as function of $z_{3}=z$ for different multipoles 

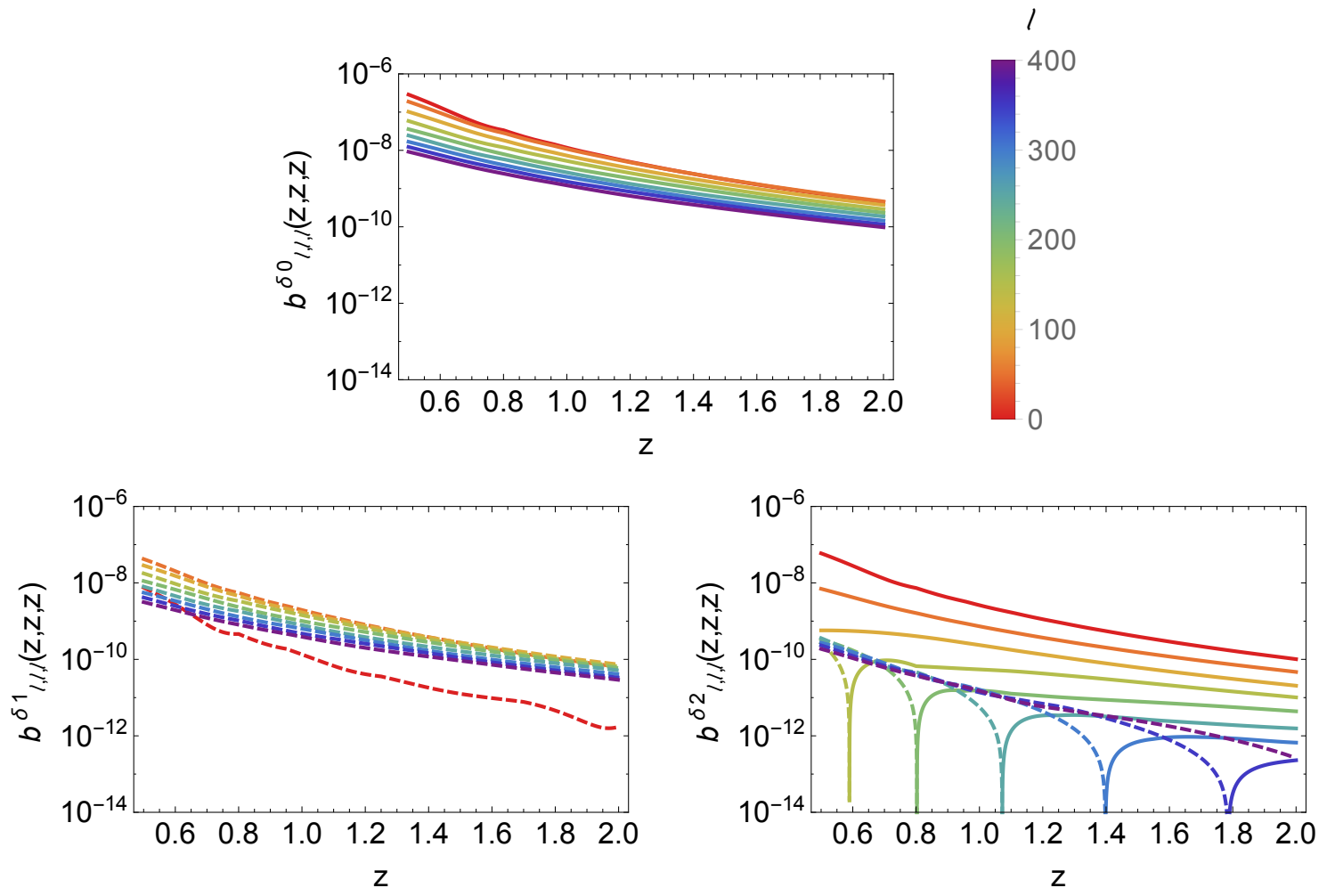

Figure 2. The monopole, dipole and quadrupole contributions to the reduced bispectrum for $\ell_{1}=\ell_{2}=\ell_{3}=\ell$ as functions of $z_{1}=z_{2}=z_{3}=z$ are shown. Different colors correspond to values from $\ell=4$ (red) to $\ell=404$ (purple) with steps $\Delta \ell=50$. Dashed lines correspond to negative values. For a fixed redshift, the dipole shows a turnover at the lower values of $\ell$ (red and orange lines). The quadrupole becomes negative at large $\ell$ 's and low redshifts.

$\ell=\ell_{1}=\ell_{2}=\ell_{3}$ indicated by the color coding. For better visibility we do not multiply them by $\ell^{2}$. The $\delta^{(2) 0}$ (top panel, we consider only the monopole part of $\delta^{(2)}$ for simplicity) and the $v^{\prime} \delta$ (low right) terms are positive at equal redshifts and dominate the result. They are of order $10^{-8}$. Also the pure RSD term (middle left) has nearly the same amplitude. The $v v^{\prime \prime}$ and $v \delta^{\prime}$ terms actually vanish exactly at equal redshifts, $z_{1}=z_{2}=z_{3}$ because of the different parity of the spherical Bessel functions which appear in the expansion of these terms, see Appendices A.2 and A.4. At slightly different redshifts they are negative and of the same order of magnitude as the other terms. Most of the terms have several sign changes especially at higher $\ell$ 's which are visible as the purple spikes in the figures. These oscillations arise from the fact that a fixed angular scale $\ell$ denotes different comoving scales $r(z)$ at different redshifts. All terms peak at $z_{3} \simeq 1$ and decay rapidly with increasing redshift difference. The same behavior has also been found for different configurations in $\ell$-space.

In Fig. 5 we show terms which contain products of Newtonian and lensing terms in the same configuration $\left(z_{i}, \ell_{i}\right)$. The top left panel is the 2 nd term in the second line of Eq. (3.8), explicitly given in (3.32). The top right panel is the 4th term in the second line of Eq. (3.8), explicitly given in (3.33). The bottom left panel is the 3rd term in the second line of Eq. (3.8), explicitly given in (3.35), and the bottom right panel is the 5th term in the second line of Eq. (3.8), explicitly given in (3.36). Also here we cannot show the 

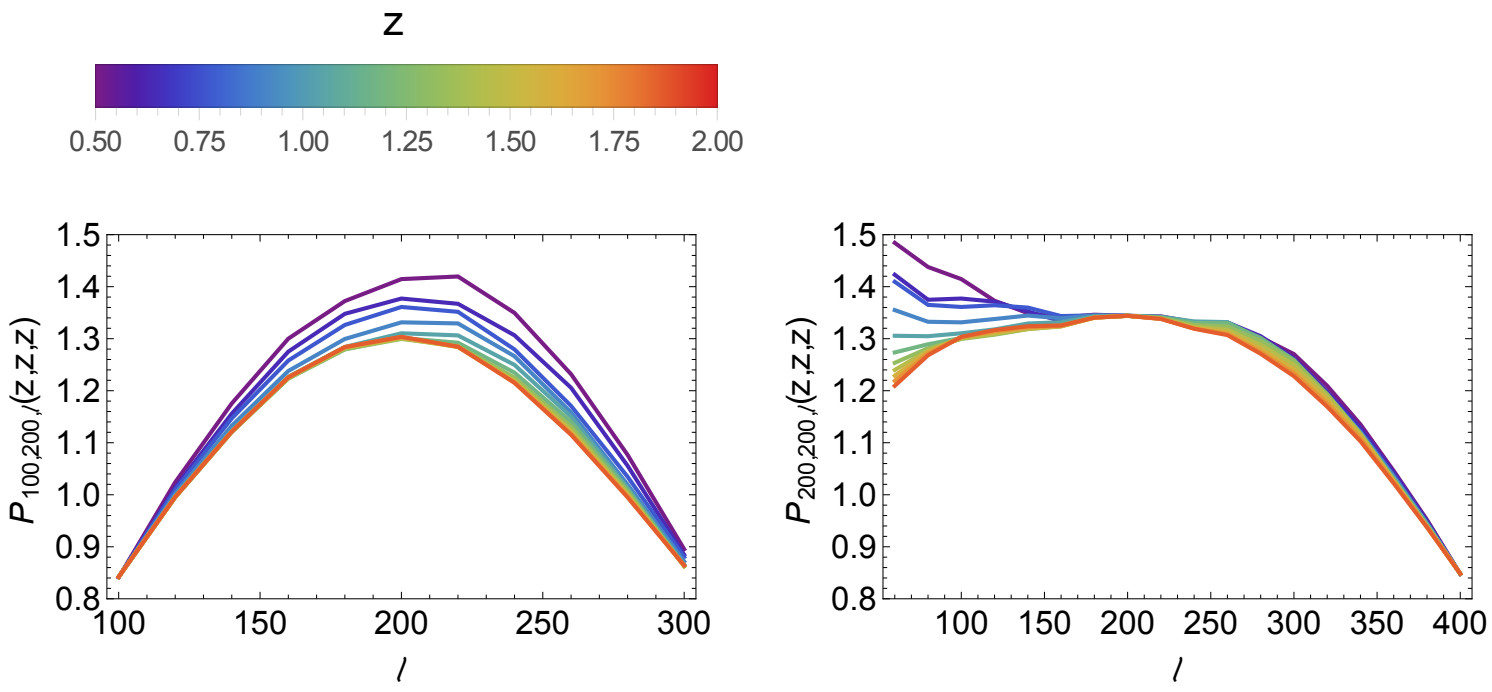

Figure 3. We plot $P_{\ell_{1} \ell_{2} \ell_{3}}\left(z_{1}, z_{2}, z_{3}\right) \equiv 1+\left(b_{\ell_{1} \ell_{2} \ell_{3}}^{v^{\prime} 1}+b_{\ell_{1} \ell_{2} \ell_{3}}^{v^{\prime} 2}\right) / b_{\ell_{1} \ell_{2} \ell_{3}}^{v^{\prime} 0}$. Different lines denote different redshift from $z=0.5$ (purple) to $z=2$ (red). The deviation from the monopole increases at low redshift, because non-Gaussianities generated by non-linearity evolution grow in time. This calculation uses the Limber approximation which cannot be assumed to be very accurate for this term.

$b_{\ell_{1} \ell_{2} \ell_{3}}^{\kappa^{(2)}}$ contribution which we have computed in the Limber approximation, see Eq. (3.57). Because of this approximation it has contact term (Dirac delta) and cannot be plotted in the configuration of Fig. 5, where no window function is included. These terms are typically of order $10^{-11}$, hence for equal redshifts they are about 3 orders of magnitude smaller than the Newtonian contributions and most probably not measurable. But at different redshifts these terms do not decay significantly with increasing redshift difference as long as $z_{3}<1=z_{1,2}$. At $z_{3}<1$, the terms containing $\kappa$ (top panels) are negative while the terms with derivatives (bottom panels) are positive. Note also that we should not trust our results for the very lowest $\ell=4$ since on this large angular scale also subdominant terms which we have not taken into account can become important.

In Fig. 6 we show the contribution of the pure lensing terms to the bispectrum. The top left panel is the 6th term in the second line of Eq. (3.8), explicitly given in (3.34). The top right panel is the 7th term in the second line of Eq. (3.8), explicitly given in (3.37). The bottom left panel is the 8th term in the second line of Eq. (3.8), explicitly given in (3.38), and the bottom right panel is the 9th term in the second line of Eq. (3.8), explicitly given in (3.39). These terms are of order $10^{-15}$ and smaller and therefore certainly unmeasurable at equal redshifts. The terms in the top panels, $\kappa^{2}$ and $\nabla_{a} \kappa \nabla^{a} \psi$, have opposite sign and for three equal $\ell$ 's they exactly cancel.

On the other hand, for different, sufficiently large $\ell$ 's and large enough redshift separations the total contribution from these pure lensing terms can actually dominate the result as can be seen in Fig. $7^{3}$. In particular, for the configuration $\ell=\ell_{1}=\ell_{2}=\ell_{3} / 2$, we have

\footnotetext{
${ }^{3}$ In Fig. 7 the contributions from the second order RSD and $\kappa^{(2)}$ are not included. In fact, since we can evaluate them only in the Limber approximation (see Eqs. (3.51) and (3.57)) their contribution vanishes in the configurations shown in Fig. 7. However, even if their contribution could be numerically evaluated starting from the exact expressions they would be of the same order as the other contributions and not change the
} 

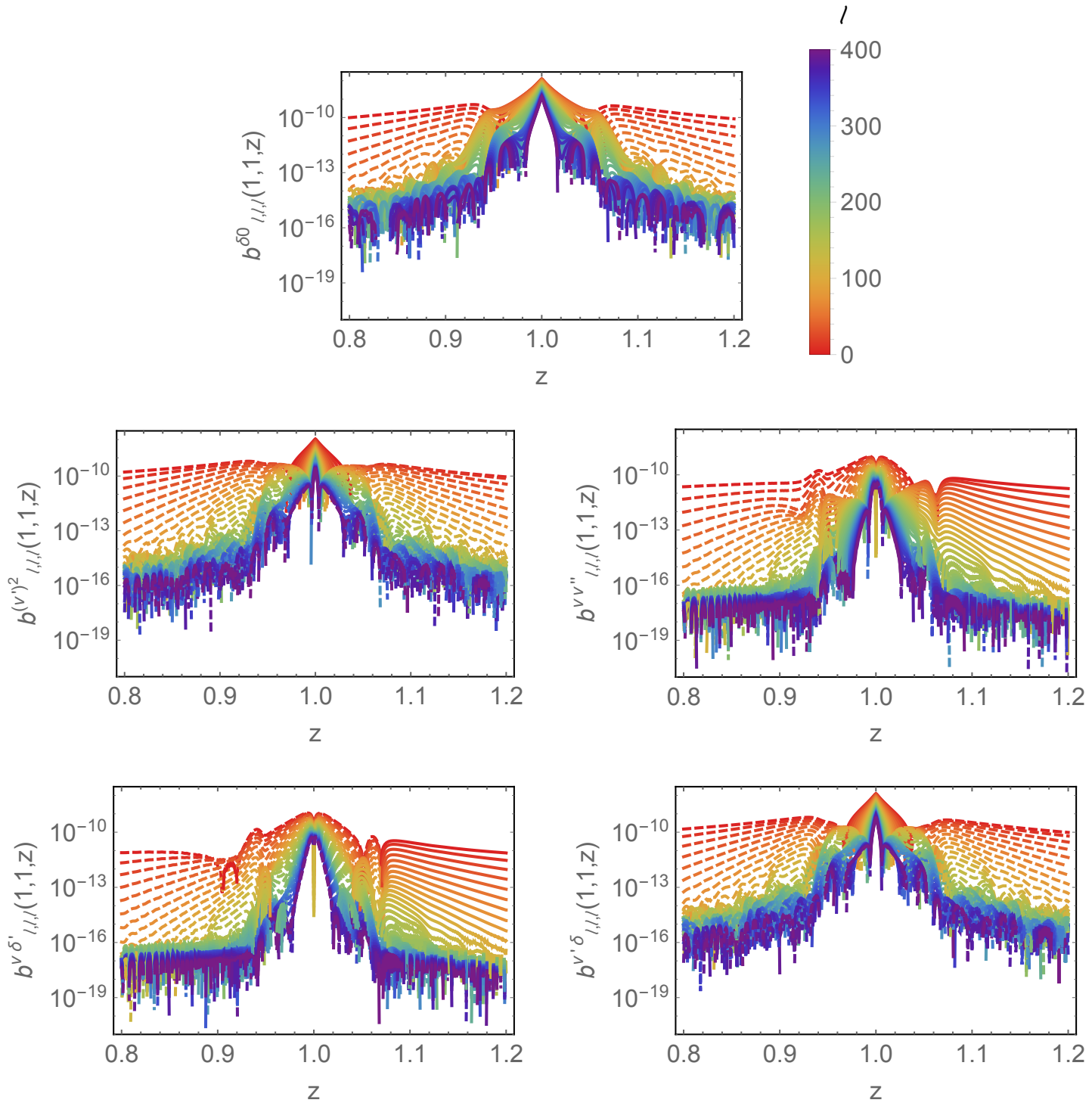

Figure 4. Newtonian terms. The contributions from the Newtonian terms to the bispectrum is shown for different values of $\ell=\ell_{1}=\ell_{2}=\ell_{3}$, from $\ell=4$ (red) to $\ell=400$ (purple), as a function of the third redshift $z_{3}=z$ for $z_{1}=z_{2}=1$. The first panel shows the first term of Eq. (3.8) while the subsequent pales show the 5 th to 8 th terms.

found that for three different redshifts $z_{1}+\Delta z=z_{2}=z_{3}-\Delta z$ with $z_{2}=1$ and $\Delta z=0.1$, the combinations of Newtonian $\times$ lensing terms dominates for $30<\ell<120$, while at larger $\ell$ 's the pure lensing terms dominate, see top-right panel of Fig. 7. For $\Delta z=0.5$ the pure lensing terms always dominate, see bottom panel of Fig. 7.

Furthermore, in analogy with the case of the power spectrum [27], in a fully tomographic analysis where all bin cross-correlations are taken into account, the signal coming from each triplet of redshift bins will add up. Distant correlations (i.e., when bins are separated by more than about $150 h^{-1} \mathrm{Mpc}$, and local terms are thus suppressed) are dominated by lensing

conclusion drawn from Fig. 7 . 

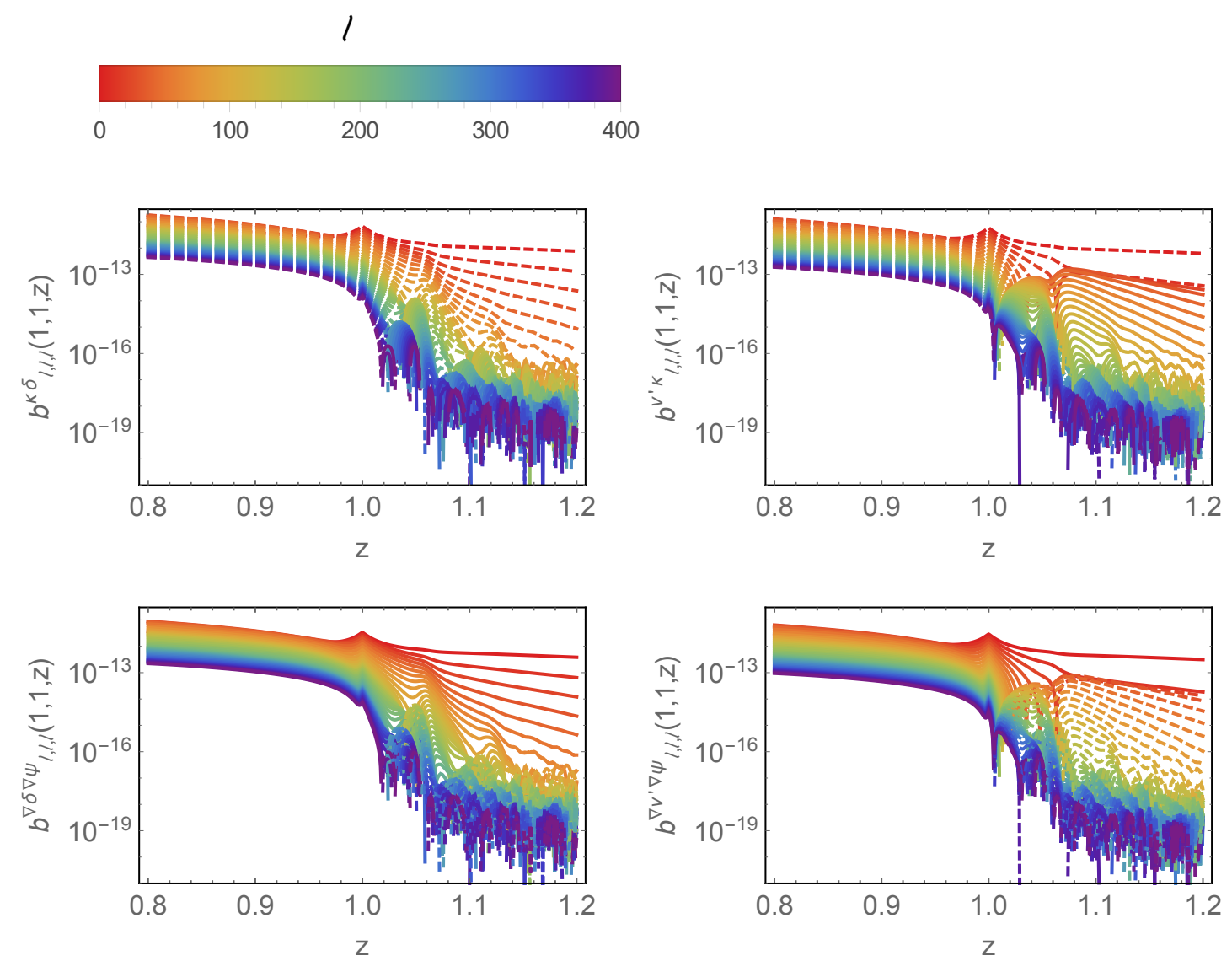

Figure 5. Newtonian $\times$ lensing terms. We plot the contributions from the 'Newtonian $\times$ lensing' terms to the bispectrum for different values of $\ell=\ell_{1}=\ell_{2}=\ell_{3}$, from $\ell=4$ (red) to $\ell=400$ (purple), as a function of the third redshift $z_{3}=z$ for $z_{1}=z_{2}=1$. These are the 2nd (top left) and 4th (top right) and the 3rd (bottom left) and 5th (bottom right) terms in the second line of Eq. (3.8).

terms. This observation shows that these new lensing terms are in principle observable in a tomographic bispectrum.

Let us finally point out that also if we consider magnification bias in the configuration for which all the pure lensing contributions vanish (see Appendix B), part of the Newtonian $\times$ lensing contribution survives and still dominates over the Newtonian terms for large enough redshift separations.

In the present study we are not considering any specific survey including noise, and hence we cannot tell whether the signal is truly measurable in a given survey. We leave this important question for a forthcoming more detailed analysis.

\subsection{Window functions}

A real observation can measure the galaxy positions only with a finite resolution. This requires the introduction of window functions in redshift space in order to compare our results with observations. One also has to introduce a binning in redshift space in order to beat shot-noise, which is inversely proportional to the number of galaxies in the bin. Once we convolve the signal with a window function we can no longer resolve scales in radial direction 

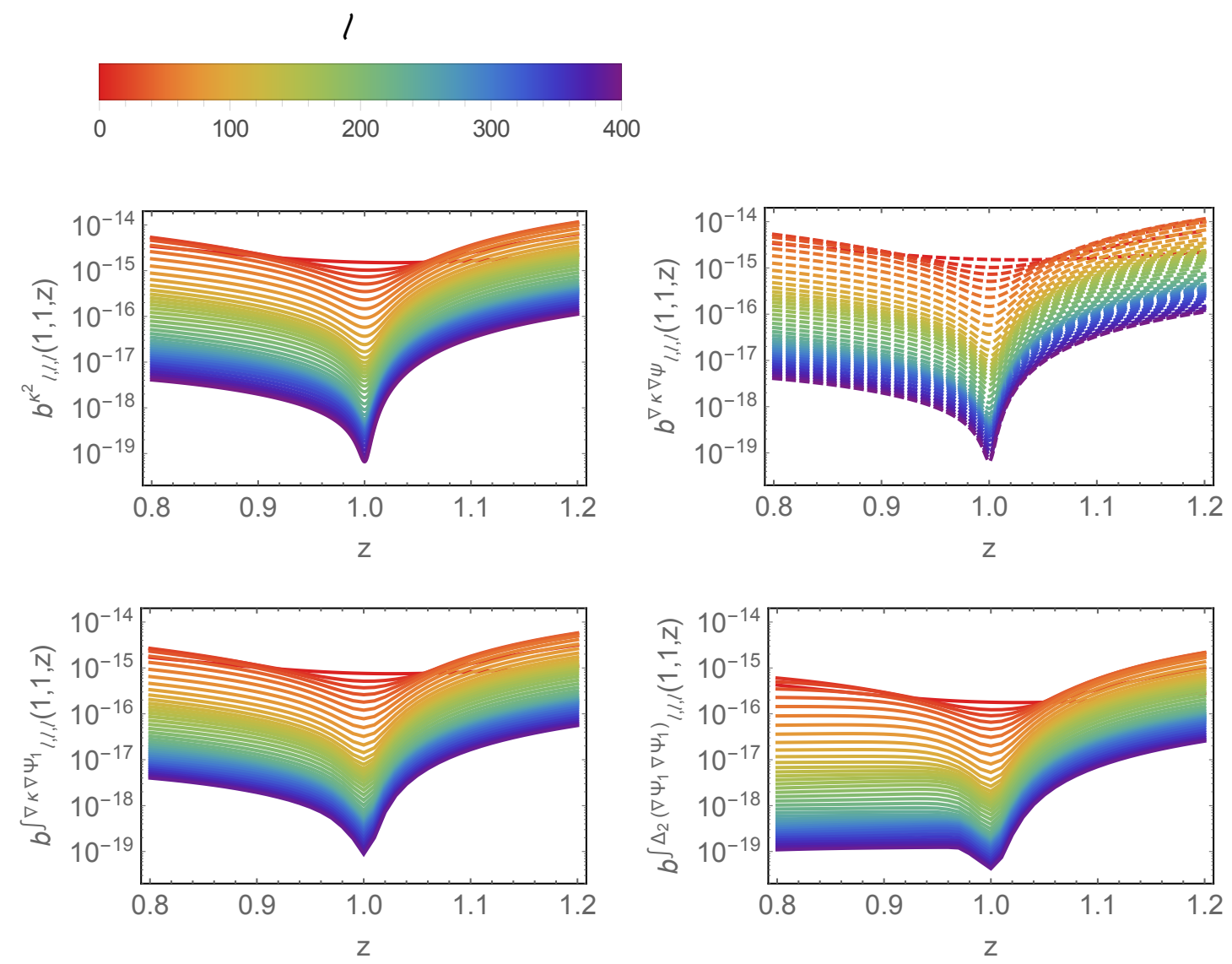

Figure 6. Lensing terms. We plot the contributions from the pure lensing terms to the bispectrum for different values of $\ell=\ell_{1}=\ell_{2}=\ell_{3}$, from $\ell=4$ (red) to $\ell=400$ (purple), as a function of the third redshift $z_{3}=z$ for $z_{1}=z_{2}=1$. These are the 6th (top left), 7th (top right), 8th (bottom left) and 9 th (bottom right) terms on the second line of Eq. (3.8). The two terms in the top panels cancel exactly for $\ell_{1}=\ell_{2}=\ell_{3}$.

which are smaller than its width. This leads to a degradation, especially, of the velocity but also of the density terms. On the other hand integrated lensing-like terms are unaffected, as it has been already shown in the past, see e.g. [13, 42] for the angular power spectrum.

We define the observed reduced bispectrum as

$$
b_{\ell_{1} \ell_{2} \ell_{3}}^{W}\left(z_{1}, z_{2}, z_{3}\right)=\int d z_{1}^{\prime} d z_{2}^{\prime} d z_{3}^{\prime} b_{\ell_{1} \ell_{2} \ell_{3}}\left(z_{1}^{\prime}, z_{2}^{\prime}, z_{3}^{\prime}\right) W\left(z_{1}, z_{1}^{\prime}\right) W\left(z_{2}, z_{2}^{\prime}\right) W\left(z_{3}, z_{3}^{\prime}\right)
$$

where $W\left(z, z^{\prime}\right)$ denotes a window function centered at $\mathrm{z}$, with an integral over $z^{\prime}$ normalized to unity. We simplify the observed reduced bispectrum (4.1) by noticing that we can express all the reduced bispectra in terms of products of angular power spectra, except for the second order terms $\delta^{(2)}, v^{(2)}$ and $\kappa^{(2)}$ which involve the kernels $F_{2}$ and $G_{2}$. For instance, most of 

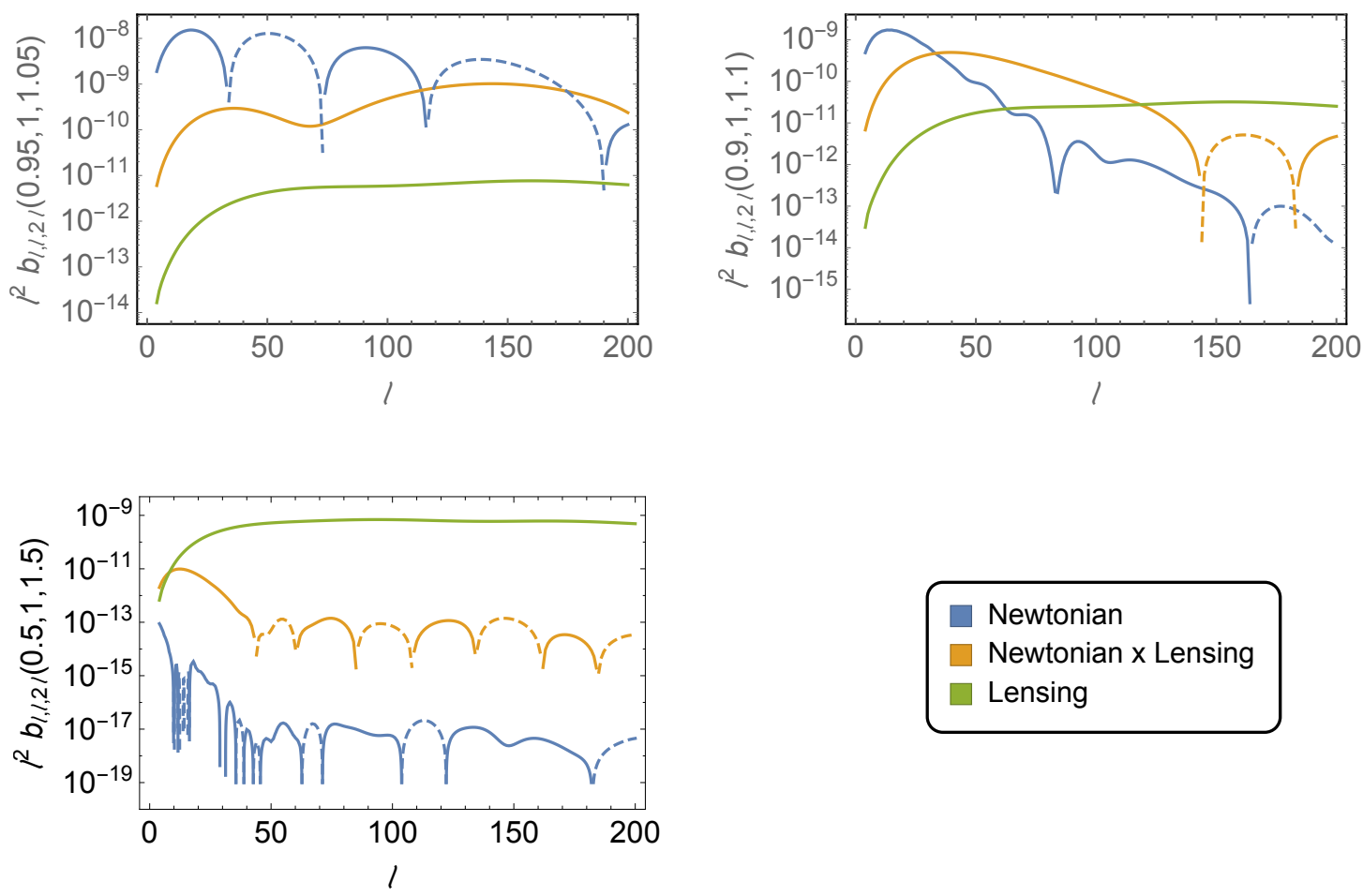

Newtonian

Newtonian $x$ Lensing

Lensing

Figure 7. Comparison. We plot the contributions from the Newtonian terms (blue), the Newtonian $\times$ lensing terms (yellow) and the pure lensing terms (green) for $z_{1}=0.95, z_{2}=1$ and $z_{3}=1.05$ (top left), for $z_{1}=0.9, z_{2}=1$ and $z_{3}=1.1$ (top right) and for $z_{1}=0.5, z_{2}=1$ and $z_{3}=1.5$ (bottom) as function of $\ell=\ell_{1}=\ell_{2}=\ell_{3} / 2$. Dashed lines correspond to negative values. The lensing terms increase with increasing redshift separation from $10^{-12}$ to $10^{-9}$, while the Newtonian terms decay from $10^{-8}$ to $10^{-17}$ and the Newtonian $\times$ lensing terms from $10^{-9}$ to $10^{-13}$. For large redshift separations the contribution from lensing clearly dominates. Furthermore, due to its integral nature, it does not show oscillations as function of $\ell$.

our contributions are of the form

$$
\begin{aligned}
b_{\ell_{1} \ell_{2} \ell_{3}}^{p_{\alpha} p_{\beta}}\left(z_{1}, z_{2}, z_{3}\right)= & c_{\ell_{2}}^{p_{\alpha} \delta}\left(z_{1}, z_{2}\right) c_{\ell_{3}}^{p_{\beta} \delta}\left(z_{1}, z_{3}\right)+c_{\ell_{3}}^{p_{\alpha} \delta}\left(z_{1}, z_{3}\right) c_{\ell_{2}}^{p_{\beta} \delta}\left(z_{1}, z_{2}\right) \\
& +c_{\ell_{1}}^{p_{\alpha} \delta}\left(z_{2}, z_{1}\right) c_{\ell_{3}}^{p_{\beta} \delta}\left(z_{2}, z_{3}\right)+c_{\ell_{3}}^{p_{\alpha} \delta}\left(z_{2}, z_{3}\right) c_{\ell_{1}}^{p_{\beta} \delta}\left(z_{2}, z_{1}\right) \\
& +c_{\ell_{1}}^{p_{\alpha} \delta}\left(z_{3}, z_{1}\right) c_{\ell_{2}}^{p_{\beta} \delta}\left(z_{3}, z_{2}\right)+c_{\ell_{2}}^{p_{\alpha} \delta}\left(z_{3}, z_{2}\right) c_{\ell_{1}}^{p_{\beta} \delta}\left(z_{3}, z_{1}\right),
\end{aligned}
$$

where $p_{\alpha}$ and $p_{\beta}$ denote any perturbation present in Eq. (2.13). For such terms we can reduce the above triple integral to a double integral. Indeed, defining

$$
\bar{c}_{\ell}^{p_{\alpha} \delta}\left(z_{i}^{\prime}, z_{j}\right) \equiv \int d z^{\prime} c_{\ell}^{p_{\alpha} \delta}\left(z_{i}^{\prime}, z^{\prime}\right) W\left(z_{j}, z^{\prime}\right)
$$


the observed reduced bispectrum can be written as

$$
\begin{aligned}
b_{\ell_{1} \ell_{2} \ell_{3}}^{W}\left(z_{1}, z_{2}, z_{3}\right)= & \int d z_{1}^{\prime} W\left(z_{1}, z_{1}^{\prime}\right)\left[\bar{c}_{\ell_{2}}^{p_{\alpha} \delta}\left(z_{1}^{\prime}, z_{2}\right) \bar{c}_{\ell_{3}}^{p_{\beta} \delta}\left(z_{1}^{\prime}, z_{3}\right)+\bar{c}_{\ell_{3}}^{p_{\alpha_{3}} \delta}\left(z_{1}^{\prime}, z_{3}\right) \bar{c}_{\ell_{2}}^{p_{\beta} \delta}\left(z_{1}^{\prime}, z_{2}\right)\right] \\
& +\int d z_{2}^{\prime} W\left(z_{2}, z_{2}^{\prime}\right)\left[\bar{c}_{\ell_{1}}^{p_{\alpha} \delta}\left(z_{2}^{\prime}, z_{1}\right) \bar{c}_{\ell_{3}}^{p_{\beta} \delta}\left(z_{2}^{\prime}, z_{3}\right)+\bar{c}_{\ell_{3}}^{p_{\alpha} \delta}\left(z_{2}^{\prime}, z_{3}\right) \bar{c}_{\ell_{1}}^{p_{\beta} \delta}\left(z_{2}^{\prime}, z_{1}\right)\right] \\
& +\int d z_{3}^{\prime} W\left(z_{3}, z_{3}^{\prime}\right)\left[\bar{c}_{\ell_{1}}^{p_{\alpha} \delta}\left(z_{3}^{\prime}, z_{1}\right) \bar{c}_{\ell_{2}}^{p_{\beta} \delta}\left(z_{3}^{\prime}, z_{2}\right)+\bar{c}_{\ell_{2}}^{p_{\alpha} \delta}\left(z_{3}^{\prime}, z_{2}\right) \bar{c}_{\ell_{1}}^{p_{\beta} \delta}\left(z_{3}^{\prime}, z_{1}\right)\right],
\end{aligned}
$$

This approach works for all the terms, except for the pure second order contributions and the integrals of products of power spectra. For the latter case it can be easily generalized. Nevertheless, for all the terms that cannot be written as a product of power spectra, we evaluate the contributions to the observed reduced bispectrum with the Limber approximation. We have tested the Limber approximation for the generalized power spectra $c_{\ell}^{A B}$ in several cases and it was always excellent (see also Fig. 9 in Appendix A.8).

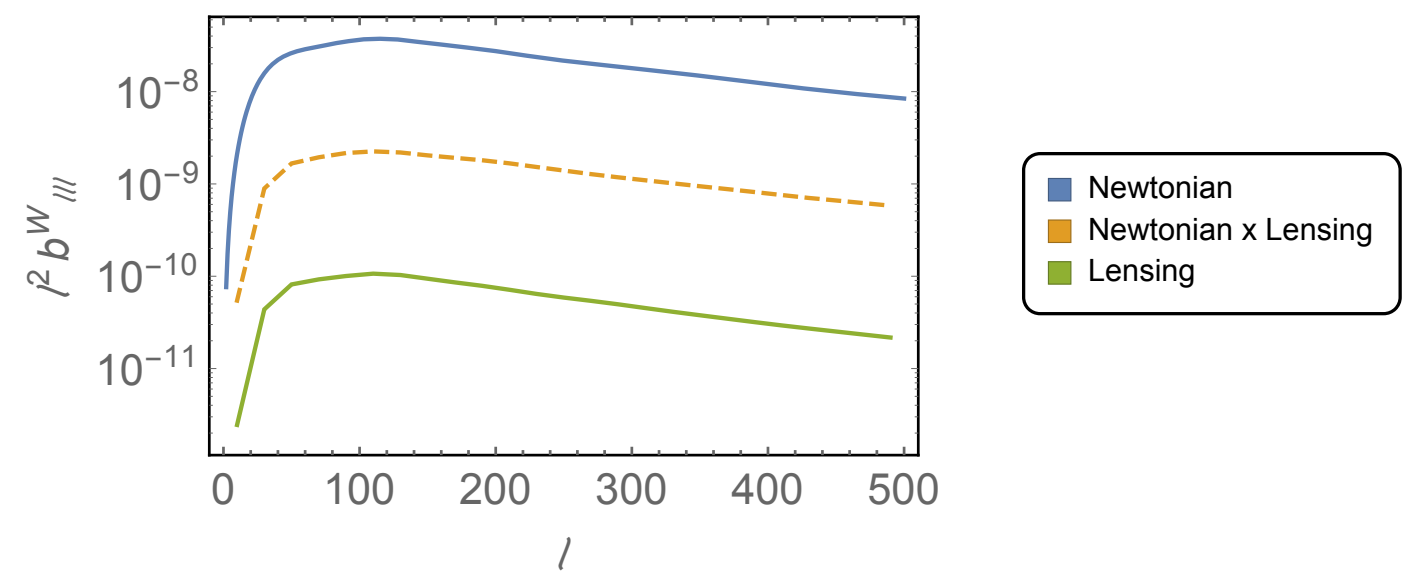

Figure 8. Window Function. We plot the contributions to the bispectrum with window function of width $\Delta z=1$ and mean redshift $z=1$, for Newtonian (blue), Newtonian $\times$ lensing (yellow) and Lensing (green). Dashed lines correspond to negative values.

In Fig. 8 we show the bispectrum with a top-hat window function of total width $\Delta z=1$ and mean redshift $z=1$. The density and especially the redshift space distortion terms are significantly reduced by this broad window, while the amplitudes of lensing terms are practically unchanged. Nevertheless, also in this case, the Newtonian terms are still nearly 2 or more orders of magnitude larger than the lensing contributions, but only one order of magnitude larger of the Newtonian $\times$ lensing terms, which are less affected by the broad window. Therefore, if the data is too sparse for a full tomographic determination of the bispectrum, integration over redshift may help to enhance the lensing contributions.

\section{Conclusions}

In this work we have calculated all the 15 terms in the bispectrum of the number counts coming from second order perturbation theory which dominate on sub-Hubble scales and at 
intermediate to large redshift. We have reproduced the 6 well known Newtonian terms and formulated them in the directly observable spherical-harmonics-redshift space. In addition we have found 9 new terms which are due to lensing by foreground structures. All these terms have to be modeled precisely and subtracted if one wants to determine a primordial non-Gaussianity from inflation by measuring the bispectrum.

On the other hand, we have seen that these lensing-like terms contain significant interesting information and it will be fascinating to measure them as they are sensitive to non-linear aspects of gravity. In this paper we provide a first inventory of all the dominant terms. We express the bispectrum in terms of 12 contributions which can be given in terms of products of power spectra and three intrinsically second order contributions which are more involved. We also show and discuss some numerical examples where we find that, like for the power spectrum, the bispectrum at equal redshifts is dominated by the density and redshift space distortion terms, which we call the Newtonian terms, while at well separated redshifts the lensing contributions dominate. We have found that for the case of three different redshifts separated by $\Delta z=0.1$, the combinations of lensing terms with density and redshift space distortion dominates for $30<\ell<120$, while at larger $\ell$ 's or for a larger redshift separation the pure lensing terms dominate, see Fig. 7.

Inspired from the expressions with the Limber approximation, we can summarise the results by saying that for three equal redshifts the Newtonian terms dominate, while for two equal redshifts the Newtonian $\times$ lensing terms dominate and for three different redshifts the pure lensing terms dominate.

Finally, we have presented some configurations of the bispectrum including a top-hat window function of total width $\Delta z=1$. While the density and, especially, the redshift space distortion terms are significantly reduced by this broad window, the amplitude of lensing terms is unchanged. Despite this reduction, the Newtonian terms are still more than two orders of magnitude larger than the pure lensing contributions, but only one order of magnitude larger than the combinations of lensing terms with density and redshift space distortion. As they are negative they lead to a decrease by about $10 \%$ of the redshift integrated bispectrum. Either this, or the especially promising method of redshift separation present opportunities to measure the Newtonian $\times$ lensing terms or even the pure lensing terms in future surveys.

As a next step we will study how to measure this bispectrum in upcoming surveys. It will be especially interesting to investigate whether planned surveys are sensitive to the lensing contributions. Even though these are always small, we have seen that they dominate the signal for sufficiently large redshift separations.

It will also be interesting to test our perturbative results with N-body simulations. For the terms presented here Newtonian N-body simulations are sufficient, but one will have to go beyond the Born approximation in ray-tracing to see all our new lensing-like terms. Present ray-tracing codes use the Born approximation which calculates the lensing potential [43, 44], but do not include this in the description of the density fluctuations on the light-cone. To go beyond the Born approximation, one will have to modify existing N-body codes either by including effects of General Relativity, see [45, 46], or by going to higher order in the photon propagation in a Newtonian N-body code.

\section{Acknowledgement}

We thank David Goldberg, David Spergel and Filippo Vernizzi for useful discussions. We are also grateful to Julien Lesgourgues and Thomas Tram for suggestions about the imple- 
mentation of some transfer function in the CLASS code. ED is supported by the ERC grant 'cosmoIGM' and by INFN/PD51 INDARK grant. GM is supported by the Marie Curie IEF, Project NeBRiC - "Non-linear effects and backreaction in classical and quantum cosmology". This work is supported by the Swiss National Science Foundation.

\section{A Derivation of bispectrum terms}

In this appendix we derive in some detail the expressions for the different contributions to the bispectrum using the formalism outlined in the main text, Section 3.1.

\section{A.1 Density term}

In this section we follow closely [47]. We denote the $n$-order density perturbation and its Fourier transform by

$$
\delta^{(n)}(\hat{\mathbf{x}}, z)=\frac{1}{(2 \pi)^{3}} \int d^{3} k \delta^{(n)}(\mathbf{k}, z) e^{-i \mathbf{k} \cdot \mathbf{x}} .
$$

In an Einstein-de Sitter universe, the second order term is given by Eq. (2.22), see [10]. We expand the mode-coupling term $F_{2}\left(\mathbf{k}_{1}, \mathbf{k}_{2}\right)$ in Legendre polynomials:

$$
F_{2}\left(\mathbf{k}_{1}, \mathbf{k}_{2}\right)=\sum_{\ell=0}^{2} f_{\ell}\left(k_{1}, k_{2}\right) P_{\ell}\left(\hat{\mathbf{k}}_{1} \cdot \hat{\mathbf{k}}_{2}\right)=\sum_{\ell m} \frac{4 \pi}{2 \ell+1} f_{\ell}\left(k_{1}, k_{2}\right) Y_{\ell m}\left(\hat{\mathbf{k}}_{1}\right) Y_{\ell m}^{*}\left(\hat{\mathbf{k}}_{2}\right)
$$

where the only non-vanishing coefficients are the monopole $f_{0}\left(k_{1}, k_{2}\right)=\frac{17}{21}$, the dipole $f_{1}\left(k_{1}, k_{2}\right)=\frac{1}{2}\left(\frac{k_{1}}{k_{2}}+\frac{k_{2}}{k_{1}}\right)$ and the quadrupole $f_{2}\left(k_{1}, k_{2}\right)=\frac{4}{21}$, given in Eq. (2.25). The linear harmonic coefficients of $\delta$ are $^{4}$

$$
a_{\ell_{1} m_{1}}^{(1)}=4 \pi i^{\ell_{1}} \int \frac{d^{3} k}{(2 \pi)^{3}} \delta^{(1)}(\mathbf{k}, z) Y_{\ell_{1} m_{1}}^{*}(\hat{\mathbf{k}}) j_{\ell_{1}}(k r)
$$

while at second order we have

$$
\begin{aligned}
a_{\ell_{3} m_{3}}^{(2)}= & \frac{(4 \pi)^{3}}{(2 \pi)^{6}} \int d k_{1} d k_{2} k_{1}^{2} k_{2}^{2} \sum_{\ell \ell^{\prime} \ell^{\prime \prime}} i^{\ell^{\prime}+\ell^{\prime \prime}} \frac{1}{2 \ell+1} f_{\ell}\left(k_{1}, k_{2}\right) j_{\ell^{\prime}}\left(k_{1} r\right) j_{\ell^{\prime \prime}}\left(k_{2} r\right) \\
& \times \int d \mathbf{n} d \hat{\mathbf{k}}_{1} d \hat{\mathbf{k}}_{2} Y_{\ell_{3} m_{3}}^{*}(\mathbf{n}) Y_{\ell^{\prime} m^{\prime}}(\mathbf{n}) Y_{\ell^{\prime \prime} m^{\prime \prime}}(\mathbf{n}) Y_{\ell^{\prime} m^{\prime}}^{*}\left(\hat{\mathbf{k}}_{1}\right) Y_{\ell m}\left(\hat{\mathbf{k}}_{1}\right) Y_{\ell^{\prime \prime} m^{\prime \prime}}^{*}\left(\hat{\mathbf{k}}_{2}\right) Y_{\ell m}^{*}\left(\hat{\mathbf{k}}_{2}\right) \\
& \times \delta^{(1)}\left(\mathbf{k}_{1}, z\right) \delta^{(1)}\left(\mathbf{k}_{2}, z\right)
\end{aligned}
$$

\footnotetext{
${ }^{4}$ We recall that in our conventions $\mathbf{n}$ is the direction of propagation of photons, opposed to the direction of observation $\hat{\mathbf{x}}=-\mathbf{n}$.
} 
The coefficients of the bispectrum are given $b^{5}$

$$
\begin{aligned}
B_{\ell_{1} \ell_{2} \ell_{3}}^{m_{1} m_{2} m_{3}}= & \left\langle a_{\ell_{1} m_{1}}^{(1)}\left(z_{1}\right) a_{\ell_{2} m_{2}}^{(1)}\left(z_{2}\right) a_{\ell_{3} m_{3}}^{(2)}\left(z_{3}\right)\right\rangle_{c}+\left\langle a_{\ell_{1} m_{1}}^{(1)}\left(z_{1}\right) a_{\ell_{2} m_{2}}^{(2)}\left(z_{2}\right) a_{\ell_{3} m_{3}}^{(1)}\left(z_{3}\right)\right\rangle_{c} \\
& +\left\langle a_{\ell_{1} m_{1}}^{(2)}\left(z_{1}\right) a_{\ell_{2} m_{2}}^{(1)}\left(z_{2}\right) a_{\ell_{3} m_{3}}^{(1)}\left(z_{3}\right)\right\rangle_{c} \\
= & \frac{(4 \pi)^{5}}{(2 \pi)^{12}} \iint d k_{1} d k_{2} d k d k^{\prime} k^{2} k^{\prime 2} k_{1}^{2} k_{2}^{2} \\
& \times \sum_{\ell^{\prime} \ell^{\prime \prime}} \frac{1}{2 \ell+1} f_{\ell}\left(k^{\prime}, k\right) j_{\ell^{\prime}}\left(k^{\prime} r_{3}\right) j_{\ell^{\prime \prime}}\left(k r_{3}\right) j_{\ell_{1}}\left(k_{1} r_{1}\right) j_{\ell_{2}}\left(k_{2} r_{2}\right) \\
& \times \int m^{\prime} m^{\prime \prime} \\
& \times d \mathbf{n} d \hat{\mathbf{k}}^{\prime} d \hat{\mathbf{k}} Y_{\ell m}\left(\hat{\mathbf{k}}^{\prime}\right) Y_{\ell m}^{*}(\hat{\mathbf{k}}) Y_{\ell^{\prime} m^{\prime}}(\mathbf{n}) Y_{\ell^{\prime} m^{\prime}}^{*}\left(\hat{\mathbf{k}}_{1}\right) Y_{\ell^{\prime \prime} m^{\prime \prime}}(\mathbf{n}) Y_{\ell^{\prime \prime} m^{\prime \prime}}^{*}(\hat{\mathbf{k}}) \\
& \times Y_{\ell_{1} m_{1}}^{*}\left(\hat{\mathbf{k}}_{1}\right) Y_{\ell_{2} m_{2}}^{*}\left(\hat{\mathbf{k}}_{2}\right) Y_{\ell_{3} m_{3}}^{*}(\mathbf{n}) \\
& \times i^{\ell^{\prime}+\ell^{\prime \prime}} i^{\ell_{1}+\ell_{2}}\left\langle\delta^{(1)}\left(\mathbf{k}_{1}, z_{1}\right) \delta^{(1)}\left(\mathbf{k}_{2}, z_{2}\right) \delta^{(1)}\left(\mathbf{k}^{\prime}, z_{3}\right) \delta^{(1)}\left(\mathbf{k}, z_{3}\right)\right\rangle_{c} \\
& +\operatorname{perms.},
\end{aligned}
$$

where we compute explicitly only the first of the three permutations. Wick's theorem gives three permutations for the density correlators, one of which contributes to the disconnected part. The two remaining permutations are equal as one sees by applying the Dirac delta from Eq. (3.9) to the integrals over $\mathbf{k}$ and $\mathbf{k}^{\prime}$, and then exchanging $\mathbf{k}_{1} \leftrightarrow \mathbf{k}_{2}$ together with $\ell^{\prime} \leftrightarrow \ell^{\prime \prime}$.

Let us introduce the geometrical factor $g_{\ell_{1} \ell_{2} \ell_{3}}$ relating the reduced bispectrum $b_{\ell_{1} \ell_{2} \ell_{3}}$, Eq. (3.4), to the angle-averaged bispectrum

$$
\begin{aligned}
g_{\ell_{1} \ell_{2} \ell_{3}} b_{\ell_{1} \ell_{2} \ell_{3}} & \equiv \sqrt{\frac{\left(2 \ell_{1}+1\right)\left(2 \ell_{2}+1\right)\left(2 \ell_{3}+1\right)}{4 \pi}}\left(\begin{array}{ccc}
\ell_{1} & \ell_{2} & \ell_{3} \\
0 & 0 & 0
\end{array}\right) b_{\ell_{1} \ell_{2} \ell_{3}} \\
& =\sum_{m_{1} m_{2} m_{3}}\left(\begin{array}{ccc}
\ell_{1} & \ell_{2} & \ell_{3} \\
m_{1} & m_{2} & m_{3}
\end{array}\right) B_{\ell_{1} \ell_{2} \ell_{3}}^{m_{1} m_{2} m_{3}}
\end{aligned}
$$

With this we obtain:

$$
\begin{aligned}
& g_{\ell_{1} \ell_{2} \ell_{3}} b_{\ell_{1} \ell_{2} \ell_{3}}=2 \frac{(4 \pi)^{2}}{(2 \pi)^{6}} \int d k_{1} d k_{2} k_{1}^{2} k_{2}^{2} P_{\mathcal{R}}\left(k_{1}\right) P_{\mathcal{R}}\left(k_{2}\right) T_{\delta}\left(k_{1}, z_{1}\right) T_{\delta}\left(k_{1}, z_{3}\right) T_{\delta}\left(k_{2}, z_{2}\right) T_{\delta}\left(k_{2}, z_{3}\right) \\
& \times \sum_{\ell^{\prime} \ell^{\prime \prime}}\left(2 \ell^{\prime}+1\right)\left(2 \ell^{\prime \prime}+1\right) j_{\ell^{\prime}}\left(k_{2} r_{3}\right) j_{\ell^{\prime \prime}}\left(k_{1} r_{3}\right) j_{\ell_{1}}\left(k_{1} r_{1}\right) j_{\ell_{2}}\left(k_{2} r_{2}\right) \\
& \times i^{\ell^{\prime}+\ell^{\prime \prime}}(-i)^{\ell_{1}+\ell_{2}}\left[\frac{17}{21} Q_{0}^{\ell_{1} \ell_{2} \ell_{3} \ell^{\prime \prime}}+\frac{1}{2}\left(\frac{k_{1}}{k_{2}}+\frac{k_{2}}{k_{1}}\right) Q_{1}^{\ell_{1} \ell_{2} \ell_{3}} \ell^{\prime \prime}+\frac{4}{21} Q_{2}^{\ell_{1} \ell_{2} \ell^{\prime} \ell_{3}}\right] \\
& \text { +perms., }
\end{aligned}
$$

where the geometrical factors $Q_{\ell \ell^{\prime} \ell^{\prime \prime}}^{\ell_{1} \ell_{3}}$ are defined below, in Eq. (A.9). In sections A.1.1, A.1.2 and A.1.3 we write the three multipoles in a form suited for numerical evaluation, and prove the form of the density bispectrum given in the main text, Eqs. (3.19), (3.20), (3.21). The following definition will be useful:

$$
{ }^{n} c_{\ell}^{A B} \ell^{\prime}\left(z_{1}, z_{2}\right) \equiv i^{\ell-\ell^{\prime}} \frac{2}{\pi} \int d k k^{2+n} P_{\mathcal{R}}(k) T_{A}\left(k, z_{1}\right) T_{B}\left(k, z_{2}\right) j_{\ell}\left(k r_{1}\right) j_{\ell^{\prime}}\left(k r_{2}\right),
$$

${ }^{5}$ This result is in agreement with Eq. (13) of [47], except for the factors $i^{\ell^{\prime}+\ell^{\prime \prime}} i^{\ell_{1}+\ell_{2}}$ which are missing in their equation. As we show in the next sections, by symmetry these terms are real and contribute as \pm 1 . But the powers of $i$ lead to important sign differences in some of the summands and cannot be ignored. 
with the convention that, on the left hand side, the first redshift $\left(z_{1}\right)$ and multipole $(\ell)$ refer to the first transfer in the superscript $(A)$, and correspondingly for the second labels. We also indicate $c_{\ell}^{A B}\left(z_{1}, z_{2}\right) \equiv{ }^{0} c_{\ell \ell}^{A B}\left(z_{1}, z_{2}\right)$. For efficient and accurate integration of the transfer functions obtained with the CLASS code ${ }^{6}$ we rely on the GNU Scientific Library. ${ }^{7}$

In Eq. (A.7) we have introduced the following integral describing the geometry of the bispectrum:

$$
\begin{aligned}
& Q_{\ell \ell^{\prime} \ell^{\prime \prime}}^{\ell_{1} \ell_{2} \ell_{3}} \equiv \int d \hat{\mathbf{l}} d \hat{\mathbf{m}} d \hat{\mathbf{n}} P_{\ell}(\hat{l} \cdot \hat{m}) P_{\ell^{\prime}}(\hat{m} \cdot \hat{n}) P_{\ell^{\prime \prime}}(\hat{l} \cdot \hat{n}) \\
& \times \sum_{m_{1}, m_{2} m_{3}}\left(\begin{array}{ccc}
\ell_{1} & \ell_{2} & \ell_{3} \\
m_{1} & m_{2} & m_{3}
\end{array}\right) Y_{\ell_{1} m_{1}}^{*}(\hat{l}) Y_{\ell_{2} m_{2}}^{*}(\hat{m}) Y_{\ell_{3} m_{3}}^{*}(\hat{n}) \\
& =\left(\frac{4 \pi}{2 \ell+1}\right)\left(\frac{4 \pi}{2 \ell^{\prime}+1}\right)\left(\frac{4 \pi}{2 \ell^{\prime \prime}+1}\right) \sum_{m_{1}, m_{2} m_{3}}\left(\begin{array}{ccc}
\ell_{1} & \ell_{2} & \ell_{3} \\
m_{1} & m_{2} & m_{3}
\end{array}\right) \\
& \times \sum_{m, m^{\prime}, m^{\prime \prime}} \int d \hat{\mathbf{l}} d \hat{\mathbf{m}} d \hat{\mathbf{n}} Y_{\ell m}(\hat{l}) Y_{\ell m}^{*}(\hat{m}) Y_{\ell^{\prime} m^{\prime}}(\hat{m}) Y_{\ell^{\prime} m^{\prime}}^{*}(\hat{n}) Y_{\ell^{\prime \prime} m^{\prime \prime}}(\hat{n}) Y_{\ell^{\prime \prime} m^{\prime \prime}}^{*}(\hat{l}) \\
& \times Y_{\ell_{1} m_{1}}^{*}(\hat{l}) Y_{\ell_{2} m_{2}}^{*}(\hat{m}) Y_{\ell_{3} m_{3}}^{*}(\hat{n}) \\
& =I_{\ell \ell^{\prime} \ell^{\prime \prime}}^{\ell_{1} \ell_{2} \ell_{3}} \sum_{m_{1}, m_{2} m_{3}}\left(\begin{array}{ccc}
\ell_{1} & \ell_{2} & \ell_{3} \\
m_{1} & m_{2} & m_{3}
\end{array}\right) \sum_{m, m^{\prime}, m^{\prime \prime}}\left(\begin{array}{ccc}
\ell & \ell^{\prime \prime} & \ell_{1} \\
m & -m^{\prime \prime} & -m_{1}
\end{array}\right) \\
& \times\left(\begin{array}{ccc}
\ell^{\prime} & \ell & \ell_{2} \\
m^{\prime} & -m & -m_{2}
\end{array}\right)\left(\begin{array}{ccc}
\ell^{\prime \prime} & \ell^{\prime} & \ell_{3} \\
m^{\prime \prime} & -m^{\prime} & -m_{3}
\end{array}\right)(-1)^{\left(m+m^{\prime}+m^{\prime \prime}\right)},
\end{aligned}
$$

where we have used the Gaunt integral and defined

$$
\begin{aligned}
I_{\ell \ell^{\prime} \ell^{\prime \prime}}^{\ell_{1} \ell_{2} \ell_{3}} \equiv & \sqrt{(4 \pi)^{3}\left(2 \ell_{1}+1\right)\left(2 \ell_{2}+1\right)\left(2 \ell_{3}+1\right)} \\
& \times\left(\begin{array}{ccc}
\ell & \ell^{\prime \prime} & \ell_{1} \\
0 & 0 & 0
\end{array}\right)\left(\begin{array}{ccc}
\ell^{\prime} & \ell & \ell_{2} \\
0 & 0 & 0
\end{array}\right)\left(\begin{array}{ccc}
\ell^{\prime \prime} & \ell^{\prime} & \ell_{3} \\
0 & 0 & 0
\end{array}\right) .
\end{aligned}
$$

Furthermore, Eq. (A.9) can be written in terms of the Wigner $6 j$ symbol as

$$
Q_{\ell \ell^{\prime} \ell^{\prime \prime}}^{\ell_{1} \ell_{2} \ell_{3}}=I_{\ell \ell^{\prime} \ell^{\prime \prime}}^{\ell_{1} \ell_{2} \ell_{3}}\left\{\begin{array}{ccc}
\ell_{1} & \ell_{2} & \ell_{3} \\
\ell^{\prime} & \ell^{\prime \prime} & \ell
\end{array}\right\}(-1)^{\ell+\ell^{\prime}+\ell^{\prime \prime}} .
$$

A useful property is that the $6 j$ symbol is non-vanishing only if the triangle condition is satisfied by the triplets

$$
\left(\ell_{1}, \ell_{2}, \ell_{3}\right),\left(\ell_{1}, \ell^{\prime \prime}, \ell\right),\left(\ell^{\prime}, \ell_{2}, \ell\right) \text { and }\left(\ell^{\prime}, \ell^{\prime \prime}, \ell_{3}\right) .
$$

For example, for the first triplet this implies:

$$
\left|\ell_{i}-\ell_{j}\right| \leq \ell_{k} \leq \ell_{i}+\ell_{j}
$$

where $(i, j, k)$ is an arbitrary permutation of $(1,2,3)$. The non-vanishing coefficients of $Q_{\ell \ell^{\prime} \ell^{\prime \prime}}^{\ell_{1} \ell_{2} \ell_{3}}$ can be determined by using these conditions, together with those related to $I_{\ell \ell^{\prime} \ell^{\prime \prime}}^{\ell_{1} \ell_{2} \ell_{3}}$, whose $3 j$ symbols further require the following sums to be even:

$$
\ell_{1}+\ell^{\prime \prime}+\ell, \ell^{\prime}+\ell_{2}+\ell, \ell^{\prime}+\ell^{\prime \prime}+\ell_{3},
$$

\footnotetext{
${ }^{6}$ http://class-code.net/

${ }^{7}$ https://www. gnu.org/software/gsl/
} 
which also implies

$$
\ell_{1}+\ell_{2}+\ell_{3}=\text { even . }
$$

For other properties in relation with Wigner $3 j$ symbols see, e.g., [48, 49].

The geometrical factors can be efficiently computed numerically in terms of Wigner symbols with publicly available libraries, e.g., WIGXJPF ${ }^{8}[50]$.

\section{A.1.1 Monopole}

For $\ell=0$, the only non-vanishing term is:

$$
Q_{0}^{\ell_{1} \ell_{2} \ell_{2} \ell_{1}}=\sqrt{\frac{\left(2 \ell_{3}+1\right)(4 \pi)^{3}}{\left(2 \ell_{2}+1\right)\left(2 \ell_{1}+1\right)}}\left(\begin{array}{ccc}
\ell_{1} & \ell_{2} & \ell_{3} \\
0 & 0 & 0
\end{array}\right) .
$$

The reduced bispectrum of the monopole reads

$$
\begin{aligned}
b_{\ell_{1} \ell_{2} \ell_{3}}^{\delta 0}\left(z_{1}, z_{2}, z_{3}\right)= & \sqrt{\frac{4 \pi}{\left(2 \ell_{1}+1\right)\left(2 \ell_{2}+1\right)\left(2 \ell_{3}+1\right)}}\left(\begin{array}{ccc}
\ell_{1} & \ell_{2} & \ell_{3} \\
0 & 0 & 0
\end{array}\right)^{-1} B_{\ell_{1} \ell_{2} \ell_{3}}^{\delta 0} \\
= & 2 \frac{17}{21} \frac{2}{\pi} \int d k_{1} k_{1}^{2} P_{\mathcal{R}}\left(k_{1}\right) T_{\delta}\left(k_{1}, z_{1}\right) T_{\delta}\left(k_{1}, z_{3}\right) j_{\ell_{1}}\left(k_{1} r_{3}\right) j_{\ell_{1}}\left(k_{1} r_{1}\right) \\
& \times \frac{2}{\pi} \int d k_{2} k_{2}^{2} P_{\mathcal{R}}\left(k_{2}\right) T_{\delta}\left(k_{2}, z_{2}\right) T_{\delta}\left(k_{2}, z_{3}\right) j_{\ell_{2}}\left(k_{2} r_{3}\right) j_{\ell_{2}}\left(k_{2} r_{2}\right) \\
& + \text { perms. }
\end{aligned}
$$

Introducing the angular-redshift power spectra, we finally obtain:

$$
b_{\ell_{1} \ell_{2} \ell_{3}}^{\delta 0}\left(z_{1}, z_{2}, z_{3}\right)=\frac{34}{21} c_{\ell_{1}}^{\delta \delta}\left(z_{1}, z_{3}\right) c_{\ell_{2}}^{\delta \delta}\left(z_{2}, z_{3}\right)+\text { perms. . }
$$

With this we recover the result already obtained in Eq. (3.19).

\section{A.1.2 Dipole}

For $\ell=1, Q_{1}^{\ell_{1} \ell_{2} \ell_{3} \ell_{3}^{\prime \prime}}$ is zero unless

$$
\ell^{\prime}=\ell_{2} \pm 1 \quad \text { and } \quad \ell^{\prime \prime}=\ell_{1} \pm 1 .
$$

This guarantees that $i^{\ell^{\prime}+\ell^{\prime \prime}}(-i)^{\ell_{1}+\ell_{2}}= \pm 1$. The dipole reduced bispectrum reads

$$
\begin{aligned}
b_{\ell_{1} \ell_{2} \ell_{3}}^{\delta 1}\left(z_{1}, z_{2}, z_{3}\right)= & \left(g_{\ell_{1} \ell_{2} \ell_{3}}\right)^{-1} \frac{1}{16 \pi^{2}} \sum_{\ell^{\prime} \ell^{\prime \prime}}\left(2 \ell^{\prime}+1\right)\left(2 \ell^{\prime \prime}+1\right) Q_{1 \ell^{\prime} \ell^{\prime \prime}}^{\ell_{1} \ell_{2} \ell_{3}} \\
& \times\left[{ }^{1} c_{\ell^{\prime \prime} \ell_{1}}^{\delta \delta}\left(z_{3}, z_{1}\right)^{-1} c_{\ell^{\prime} \ell_{2}}^{\delta \delta}\left(z_{3}, z_{2}\right)+{ }^{-1} c_{\ell^{\prime \prime} \ell_{1}}^{\delta \delta}\left(z_{3}, z_{1}\right){ }^{1} c_{\ell^{\prime} \ell_{2}}^{\delta \delta}\left(z_{3}, z_{2}\right)\right] \\
& + \text { perms. }
\end{aligned}
$$

\section{A.1.3 Quadrupole}

If $\ell=2, Q_{2}^{\ell_{1} \ell_{2} \ell_{3}} \ell^{\prime} \ell^{\prime \prime}$ is zero unless

$$
\ell^{\prime}=\ell_{2} \pm 2, \ell_{2} \quad \text { and } \quad \ell^{\prime \prime}=\ell_{1} \pm 2, \ell_{1} .
$$

This guarantees that $i^{\ell^{\prime}+\ell^{\prime \prime}}(-i)^{\ell_{1}+\ell_{2}}= \pm 1$. The quadrupole bispectrum reads

$$
\begin{aligned}
b_{\ell_{1} \ell_{2} \ell_{3}}^{\delta 2}\left(z_{1}, z_{2}, z_{3}\right)= & \left(g_{\ell_{1} \ell_{2} \ell_{3}}\right)^{-1} \frac{1}{42 \pi^{2}} \sum_{\ell^{\prime} \ell^{\prime \prime}}\left(2 \ell^{\prime}+1\right)\left(2 \ell^{\prime \prime}+1\right) Q_{2}^{\ell_{1} \ell_{2} \ell_{3} \ell_{3}^{\prime \prime}}{ }^{0} c_{\ell^{\prime \prime} \ell_{1}}^{\delta \delta}\left(z_{3}, z_{1}\right){ }^{0} c_{\ell^{\prime} \ell_{2}}^{\delta \delta}\left(z_{3}, z_{2}\right) \\
& + \text { perms. }
\end{aligned}
$$

\footnotetext{
${ }^{8}$ http://fy.chalmers.se/subatom/wigxjpf/
} 


\section{A.2 Term $\mathcal{H}^{-2} \partial_{r} v \partial_{r}^{3} v$}

We consider

$$
\left\langle\left(\mathcal{H}^{-2} \partial_{r} v \partial_{r}^{3} v\right)\left(\mathbf{n}_{1}, z_{1}\right) \delta\left(\mathbf{n}_{2}, z_{2}\right) \delta\left(\mathbf{n}_{3}, z_{3}\right)\right\rangle_{c}+\text { perm. . }
$$

We write the first factor as a product of integrals in Fourier space,

$$
\Delta^{v v^{\prime \prime}}(\mathbf{n}, z)=\frac{\mathcal{H}^{-2}(z)}{(2 \pi)^{6}} \int d^{3} k d^{3} k_{1} k_{1}^{2} V(\mathbf{k}, \eta) V\left(\mathbf{k}_{1}, \eta\right) \partial_{(k r)} e^{i \mathbf{k} \cdot \mathbf{n} r} \partial_{\left(k_{1} r\right)}^{3} e^{i \mathbf{k}_{1} \cdot \mathbf{n} r},
$$

where $V$ is the velocity potential (in Newtonian gauge) defined through $\mathbf{v}=i \hat{\mathbf{k}} V$. Here $\eta=\eta(z)$ and $r=r(z)=\eta_{0}-\eta(z)$. We then compute (A.23)

$$
\begin{aligned}
\langle\cdots & \rangle=\frac{1}{\mathcal{H}\left(z_{1}\right)^{2}(2 \pi)^{12}} \int d^{3} k d^{3} k_{1} d^{3} k_{2} d^{3} k_{3} k_{1}^{2}\left(\partial_{\left(k r_{1}\right)} e^{i \mathbf{k} \cdot \mathbf{n}_{1} r_{1}}\right)\left(\partial_{\left(k_{1} r_{1}\right)}^{3} e^{i \mathbf{k}_{1} \cdot \mathbf{n}_{1} r_{1}}\right) e^{i\left(\mathbf{k}_{2} \cdot \mathbf{n}_{2} r_{2}+\mathbf{k}_{3} \cdot \mathbf{n}_{3} r_{3}\right)} \\
& \times T_{v}\left(k, \eta_{1}\right) T_{v}\left(k_{1}, \eta_{1}\right) T_{\delta}\left(k_{2}, \eta_{2}\right) T_{\delta}\left(k_{3}, \eta_{3}\right)\left\langle R(\mathbf{k}) R\left(\mathbf{k}_{1}\right) R\left(\mathbf{k}_{2}\right) R\left(\mathbf{k}_{3}\right)\right\rangle \\
= & \frac{1}{\mathcal{H}\left(z_{1}\right)^{2}(2 \pi)^{6}} \int d^{3} k_{2} d^{3} k_{3} k_{3}^{2}\left(\partial_{\left(k_{2} r_{1}\right)} e^{-i \mathbf{k}_{2} \cdot \mathbf{n}_{1} r_{1}}\right)\left(\partial_{\left(k_{3} r_{1}\right)}^{3} e^{-i \mathbf{k}_{3} \cdot \mathbf{n}_{1} r_{1}}\right) e^{i\left(\mathbf{k}_{2} \cdot \mathbf{n}_{2} r_{2}+\mathbf{k}_{3} \cdot \mathbf{n}_{3} r_{3}\right)} \\
& \times T_{v}\left(k_{2}, \eta_{1}\right) T_{v}\left(k_{3}, \eta_{1}\right) T_{\delta}\left(k_{2}, \eta_{2}\right) T_{\delta}\left(k_{3}, \eta_{3}\right) P_{R}\left(k_{2}\right) P_{R}\left(k_{3}\right) \\
& +\frac{1}{\mathcal{H}\left(z_{1}\right)^{2}(2 \pi)^{6}} \int d^{3} k_{2} d^{3} k_{3} k_{2}^{2}\left(\partial_{\left(k_{3} r_{1}\right)} e^{-i \mathbf{k}_{3} \cdot \mathbf{n}_{1} r_{1}}\right)\left(\partial_{\left(k_{2} r_{1}\right)}^{3} e^{-i \mathbf{k}_{2} \cdot \mathbf{n}_{1} r_{1}}\right) e^{i\left(\mathbf{k}_{2} \cdot \mathbf{n}_{2} r_{2}+\mathbf{k}_{3} \cdot \mathbf{n}_{3} r_{3}\right)} \\
& \times T_{v}\left(k_{2}, \eta_{1}\right) T_{v}\left(k_{3}, \eta_{1}\right) T_{\delta}\left(k_{2}, \eta_{2}\right) T_{\delta}\left(k_{3}, \eta_{3}\right) P_{R}\left(k_{2}\right) P_{R}\left(k_{3}\right) .
\end{aligned}
$$

Expanding the exponentials in spherical harmonics and spherical Bessel functions we obtain

$$
\begin{aligned}
B^{v v^{\prime \prime}}\left(\mathbf{n}_{1}, \mathbf{n}_{2}, \mathbf{n}_{3}, z_{1}, z_{2}, z_{3}\right)= & \frac{4}{\pi^{2}} \sum_{\substack{\ell, \ell^{\prime} \\
m, m^{\prime}}} Y_{\ell m}\left(\mathbf{n}_{1}\right) Y_{\ell^{\prime} m^{\prime}}\left(\mathbf{n}_{1}\right) Y_{\ell m}^{*}\left(\mathbf{n}_{2}\right) Y_{\ell^{\prime} m^{\prime}}^{*}\left(\mathbf{n}_{3}\right) Z_{\ell \ell^{\prime}}^{v v^{\prime \prime}}\left(z_{1}, z_{2}, z_{3}\right) \\
& + \text { perm. }
\end{aligned}
$$

where

$$
\begin{aligned}
& Z_{\ell \ell^{\prime}}^{v v^{\prime \prime}}\left(z_{1}, z_{2}, z_{3}\right) \\
& \begin{aligned}
=\frac{1}{\mathcal{H}\left(z_{1}\right)^{2}} \int d k_{2} d k_{3} k_{2}^{2} k_{3}^{4} P_{R}\left(k_{2}\right) P_{R}\left(k_{3}\right) T_{v}\left(k_{2}, \eta_{1}\right) T_{v}\left(k_{3}, \eta_{1}\right) T_{\delta}\left(k_{2}, \eta_{2}\right) T_{\delta}\left(k_{3}, \eta_{3}\right) \\
\quad \times j_{\ell}^{\prime}\left(k_{2} r_{1}\right) j_{\ell^{\prime}}^{\prime \prime \prime}\left(k_{3} r_{1}\right) j_{\ell}\left(k_{2} r_{2}\right) j_{\ell^{\prime}}\left(k_{3} r_{3}\right)
\end{aligned} \\
& \quad+\frac{1}{\mathcal{H}\left(z_{1}\right)^{2}} \int d k_{2} d k_{3} k_{2}^{4} k_{3}^{2} P_{R}\left(k_{2}\right) P_{R}\left(k_{3}\right) T_{v}\left(k_{2}, \eta_{1}\right) T_{v}\left(k_{3}, \eta_{1}\right) T_{\delta}\left(k_{2}, \eta_{2}\right) T_{\delta}\left(k_{3}, \eta_{3}\right) \\
& \quad \times j_{\ell}^{\prime \prime \prime}\left(k_{2} r_{1}\right) j_{\ell^{\prime}}^{\prime}\left(k_{3} r_{1}\right) j_{\ell}\left(k_{2} r_{2}\right) j_{\ell^{\prime}}\left(k_{3} r_{3}\right) .
\end{aligned}
$$

Note that this expression vanishes if $r_{1}=r_{2}$ or $r_{1}=r_{3}$ i.e. $z_{1}=z_{2}$ or $z_{1}=z_{3}$ due to the opposite parity of $j_{\ell}$ and $j_{\ell}^{\prime}$ as well as $j_{\ell}$ and $j_{\ell}^{\prime \prime \prime}$. The reduced bispectrum is therefore given by

$$
b_{\ell_{1} \ell_{2} \ell_{3}}^{v v^{\prime \prime}}=\frac{4}{\pi^{2}} Z_{\ell_{2} \ell_{3}}^{v v^{\prime \prime}}\left(z_{1}, z_{2}, z_{3}\right)+\text { perm.. }
$$

Due to the sum this vanishes only if all three redshifts coincide. 
Making use of the definition (3.11) and of the transfer functions in multipole space (3.22) and (3.24), we can express the bispectrum (A.28) as

$$
\begin{aligned}
b_{\ell_{1} \ell_{2} \ell_{3}}^{v v^{\prime \prime}}= & c_{\ell_{2}}^{v \delta}\left(z_{1}, z_{2}\right) c_{\ell_{3}}^{v^{\prime \prime} \delta}\left(z_{1}, z_{3}\right)+c_{\ell_{2}}^{v^{\prime \prime} \delta}\left(z_{1}, z_{2}\right) c_{\ell_{3}}^{v \delta}\left(z_{1}, z_{3}\right) \\
& +c_{\ell_{1}}^{v \delta}\left(z_{2}, z_{1}\right) c_{\ell_{3}}^{v^{\prime \prime} \delta}\left(z_{2}, z_{3}\right)+c_{\ell_{1}}^{v^{\prime \prime} \delta}\left(z_{2}, z_{1}\right) c_{\ell_{3}}^{v \delta}\left(z_{2}, z_{3}\right) \\
& +c_{\ell_{1}}^{v \delta}\left(z_{3}, z_{1}\right) c_{\ell_{2}}^{v^{\prime \prime} \delta}\left(z_{3}, z_{2}\right)+c_{\ell_{1}}^{v^{\prime \prime} \delta}\left(z_{3}, z_{1}\right) c_{\ell_{2}}^{v \delta}\left(z_{3}, z_{2}\right) .
\end{aligned}
$$

\section{A.3 Term $\mathcal{H}^{-2} \partial_{r}^{2} v \partial_{r}^{2} v$}

We compute the contribution of the following term.

$$
\left\langle\left(\mathcal{H}^{-2} \partial_{r}^{2} v \partial_{r}^{2} v\right)\left(\mathbf{n}_{1}, z_{1}\right) \delta\left(\mathbf{n}_{2}, z_{2}\right) \delta\left(\mathbf{n}_{3}, z_{3}\right)\right\rangle_{c}+\text { perm. . }
$$

In Fourier space we can rewrite the product of the redshift space distortions as

$$
\Delta^{{v^{\prime}}^{2}}(\mathbf{n}, z)=\frac{\mathcal{H}^{-2}(z)}{(2 \pi)^{6}} \int d^{3} k d^{3} k_{1} k k_{1} V(\mathbf{k}, \eta) V\left(\mathbf{k}_{1}, \eta\right) \partial_{(k r)}^{2} e^{i \mathbf{k} \cdot \mathbf{n} r} \partial_{\left(k_{1} r\right)}^{2} e^{i \mathbf{k}_{1} \cdot \mathbf{n} r}
$$

such that

$$
\begin{aligned}
\langle\cdots\rangle & =\frac{1}{\mathcal{H}\left(z_{1}\right)^{2}(2 \pi)^{12}} \int d^{3} k d^{3} k_{1} d^{3} k_{2} d^{3} k_{3} k k_{1}\left(\partial_{\left(k r_{1}\right)}^{2} e^{i \mathbf{k} \cdot \mathbf{n}_{1} r_{1}}\right)\left(\partial_{\left(k_{1} r_{1}\right)}^{2} e^{i \mathbf{k}_{1} \cdot \mathbf{n}_{1} r_{1}}\right) \\
& \times e^{i\left(\mathbf{k}_{2} \cdot \mathbf{n}_{2} r_{2}+\mathbf{k}_{3} \cdot \mathbf{n}_{3} r_{3}\right)} T_{v}\left(k, \eta_{1}\right) T_{v}\left(k_{1}, \eta_{1}\right) T_{\delta}\left(k_{2}, \eta_{2}\right) T_{\delta}\left(k_{3}, \eta_{3}\right)\left\langle R(\mathbf{k}) R\left(\mathbf{k}_{1}\right) R\left(\mathbf{k}_{2}\right) R\left(\mathbf{k}_{3}\right)\right\rangle \\
& =\frac{2}{\mathcal{H}\left(z_{1}\right)^{2}(2 \pi)^{6}} \int d^{3} k_{2} d^{3} k_{3} k_{2} k_{3}\left(\partial_{\left(k_{2} r_{1}\right)}^{2} e^{-i \mathbf{k}_{2} \cdot \mathbf{n}_{1} r_{1}}\right)\left(\partial_{\left(k_{3} r_{1}\right)}^{2} e^{-i \mathbf{k}_{3} \cdot \mathbf{n}_{1} r_{1}}\right) \\
& \times e^{i\left(\mathbf{k}_{2} \cdot \mathbf{n}_{2} r_{2}+\mathbf{k}_{3} \cdot \mathbf{n}_{3} r_{3}\right)} T_{v}\left(k_{2}, \eta_{1}\right) T_{v}\left(k_{3}, \eta_{1}\right) T_{\delta}\left(k_{2}, \eta_{2}\right) T_{\delta}\left(k_{3}, \eta_{3}\right) P_{R}\left(k_{2}\right) P_{R}\left(k_{3}\right) . \quad
\end{aligned}
$$

Again, by expanding the exponentials in spherical harmonics and spherical Bessel functions we find

$$
\begin{aligned}
B^{v^{\prime 2}}\left(\mathbf{n}_{1}, \mathbf{n}_{2}, \mathbf{n}_{3}, z_{1}, z_{2}, z_{3}\right)= & \frac{4}{\pi^{2}} \sum_{\substack{\ell, \ell^{\prime} \\
m, m^{\prime}}} Y_{\ell m}\left(\mathbf{n}_{1}\right) Y_{\ell^{\prime} m^{\prime}}\left(\mathbf{n}_{1}\right) Y_{\ell m}^{*}\left(\mathbf{n}_{2}\right) Y_{\ell^{\prime} m^{\prime}}^{*}\left(\mathbf{n}_{3}\right) Z_{\ell \ell^{\prime}}^{v^{\prime 2}}\left(z_{1}, z_{2}, z_{3}\right) \\
& + \text { perm. }
\end{aligned}
$$

where

$$
\begin{aligned}
Z_{\ell \ell^{\prime}}^{v^{\prime 2}}\left(z_{1}, z_{2}, z_{3}\right)= & \frac{2}{\mathcal{H}\left(z_{1}\right)^{2}} \int d k_{2} d k_{3} k_{2}^{3} k_{3}^{3} T_{v}\left(k_{2}, \eta_{1}\right) T_{v}\left(k_{3}, \eta_{1}\right) T_{\delta}\left(k_{2}, \eta_{2}\right) T_{\delta}\left(k_{3}, \eta_{3}\right) \\
& \quad \times P_{R}\left(k_{2}\right) P_{R}\left(k_{3}\right) j_{\ell}^{\prime \prime}\left(k_{2} r_{1}\right) j_{\ell^{\prime}}^{\prime \prime}\left(k_{3} r_{1}\right) j_{\ell}\left(k_{2} r_{2}\right) j_{\ell^{\prime}}\left(k_{3} r_{3}\right) .
\end{aligned}
$$

These leads to the reduced bispectrum

$$
b_{\ell_{1} \ell_{2} \ell_{3}}^{v^{\prime 2}}=\frac{4}{\pi^{2}} Z_{\ell_{2} \ell_{3}}^{v^{\prime 2}}\left(z_{1}, z_{2}, z_{3}\right)+\text { perm. }
$$

which can be rewritten in terms of product of angular power spectra

$$
b_{\ell_{1} \ell_{2} \ell_{3}}^{v^{\prime 2}}=2\left(c_{\ell_{2}}^{v^{\prime} \delta}\left(z_{1}, z_{2}\right) c_{\ell_{3}}^{v^{\prime} \delta}\left(z_{1}, z_{3}\right)+c_{\ell_{1}}^{v^{\prime} \delta}\left(z_{2}, z_{1}\right) c_{\ell_{3}}^{v^{\prime} \delta}\left(z_{2}, z_{3}\right)+c_{\ell_{1}}^{v^{\prime} \delta}\left(z_{3}, z_{1}\right) c_{\ell_{2}}^{v^{\prime} \delta}\left(z_{3}, z_{2}\right)\right) .
$$




\section{A.4 Term $\mathcal{H}^{-1} \partial_{r} v \partial_{r} \delta$}

We consider the term

$$
\left\langle\left(\mathcal{H}^{-1} \partial_{r} v \partial_{r} \delta\right)\left(\mathbf{n}_{1}, z_{1}\right) \delta\left(\mathbf{n}_{2}, z_{2}\right) \delta\left(\mathbf{n}_{3}, z_{3}\right)\right\rangle_{c}+\text { perm. . }
$$

Again we write the first factor as a product of Fourier integrals,

$$
\Delta^{v \delta^{\prime}}(\mathbf{n}, z)=\frac{\mathcal{H}^{-1}}{(2 \pi)^{6}} \int d^{3} k d^{3} k_{1} k_{1} V(\mathbf{k}, \eta) \delta\left(\mathbf{k}_{1}, \eta\right) \partial_{(k r)} e^{i \mathbf{k} \cdot \mathbf{n} r} \partial_{\left(k_{1} r\right)} e^{i \mathbf{k}_{1} \cdot \mathbf{n} r} .
$$

We now compute (A.37) as above by using its Fourier representation

$$
\begin{aligned}
\langle\cdots\rangle= & \frac{1}{\mathcal{H}\left(z_{1}\right)(2 \pi)^{12}} \int d^{3} k d^{3} k_{1} d^{3} k_{2} d^{3} k_{3} k_{1} \partial_{\left(k r_{1}\right)} e^{i \mathbf{k} \cdot \mathbf{n}_{1} r_{1}} \partial_{\left(k_{1} r_{1}\right)} e^{i \mathbf{k}_{1} \cdot \mathbf{n}_{1} r_{1}} e^{i\left(\mathbf{k}_{2} \cdot \mathbf{n}_{2} r_{2}+\mathbf{k}_{3} \cdot \mathbf{n}_{3} r_{3}\right)} \\
& \times T_{v}\left(k, \eta_{1}\right) T_{\delta}\left(k_{1}, \eta_{1}\right) T_{\delta}\left(k_{2}, \eta_{2}\right) T_{\delta}\left(k_{3}, \eta_{3}\right)\left\langle R(\mathbf{k}) R\left(\mathbf{k}_{1}\right) R\left(\mathbf{k}_{2}\right) R\left(\mathbf{k}_{3}\right)\right\rangle \\
= & \frac{1}{\mathcal{H}\left(z_{1}\right)(2 \pi)^{6}} \int d^{3} k_{2} d^{3} k_{3} k_{3} \partial_{\left(k_{2} r_{1}\right)} e^{-i \mathbf{k}_{2} \cdot \mathbf{n}_{1} r_{1}} \partial_{\left(k_{3} r_{1}\right)} e^{-i \mathbf{k}_{3} \cdot \mathbf{n}_{1} r_{1}} e^{i\left(\mathbf{k}_{2} \cdot \mathbf{n}_{2} r_{2}+\mathbf{k}_{3} \cdot \mathbf{n}_{3} r_{3}\right)} \\
& \times T_{v}\left(k_{2}, \eta_{1}\right) T_{\delta}\left(k_{3}, \eta_{1}\right) T_{\delta}\left(k_{2}, \eta_{2}\right) T_{\delta}\left(k_{3}, \eta_{3}\right) P_{R}\left(k_{2}\right) P_{R}\left(k_{3}\right) \\
& +\frac{1}{\mathcal{H}\left(z_{1}\right)(2 \pi)^{6}} \int d^{3} k_{2} d^{3} k_{3} k_{2} \partial_{\left(k_{3} r_{1}\right)} e^{-i \mathbf{k}_{3} \cdot \mathbf{n}_{1} r_{1}} \partial_{\left(k_{2} r_{1}\right)} e^{-i \mathbf{k}_{2} \cdot \mathbf{n}_{1} r_{1}} e^{i\left(\mathbf{k}_{2} \cdot \mathbf{n}_{2} r_{2}+\mathbf{k}_{3} \cdot \mathbf{n}_{3} r_{3}\right)} \\
& \times T_{v}\left(k_{3}, \eta_{1}\right) T_{\delta}\left(k_{2}, \eta_{1}\right) T_{\delta}\left(k_{2}, \eta_{2}\right) T_{\delta}\left(k_{3}, \eta_{3}\right) P_{R}\left(k_{2}\right) P_{R}\left(k_{3}\right)
\end{aligned}
$$

from which follows

$$
\begin{aligned}
B^{v \delta^{\prime}}\left(\mathbf{n}_{1}, \mathbf{n}_{2}, \mathbf{n}_{3}, z_{1}, z_{2}, z_{3}\right)= & \frac{4}{\pi^{2}} \sum_{\substack{\ell, \ell^{\prime} \\
m, m^{\prime}}} Y_{\ell m}\left(\mathbf{n}_{1}\right) Y_{\ell^{\prime} m^{\prime}}\left(\mathbf{n}_{1}\right) Y_{\ell m}^{*}\left(\mathbf{n}_{2}\right) Y_{\ell^{\prime} m^{\prime}}^{*}\left(\mathbf{n}_{3}\right) Z_{\ell \ell^{\prime}}^{v \delta^{\prime}}\left(z_{1}, z_{2}, z_{3}\right) \\
& + \text { perm. }
\end{aligned}
$$

where

$$
\begin{aligned}
Z_{\ell \ell^{\prime}}^{v \delta^{\prime}}\left(z_{1}, z_{2}, z_{3}\right)= & \frac{1}{\mathcal{H}\left(z_{1}\right)} \int d k_{2} d k_{3} k_{2}^{2} k_{3}^{3} T_{v}\left(k_{2}, \eta_{1}\right) T_{\delta}\left(k_{3}, \eta_{1}\right) T_{\delta}\left(k_{2}, \eta_{2}\right) T_{\delta}\left(k_{3}, \eta_{3}\right) \\
& \quad \times P_{R}\left(k_{2}\right) P_{R}\left(k_{3}\right) j_{\ell}^{\prime}\left(k_{2} r_{1}\right) j_{\ell^{\prime}}^{\prime}\left(k_{3} r_{1}\right) j_{\ell}\left(k_{2} r_{2}\right) j_{\ell^{\prime}}\left(k_{3} r_{3}\right) \\
& +\frac{1}{\mathcal{H}\left(z_{1}\right)} \int d k_{2} d k_{3} k_{2}^{3} k_{3}^{2} T_{v}\left(k_{3}, \eta_{1}\right) T_{\delta}\left(k_{2}, \eta_{1}\right) T_{\delta}\left(k_{2}, \eta_{2}\right) T_{\delta}\left(k_{3}, \eta_{3}\right) \\
& \quad \times P_{R}\left(k_{2}\right) P_{R}\left(k_{3}\right) j_{\ell}^{\prime}\left(k_{2} r_{1}\right) j_{\ell^{\prime}}^{\prime}\left(k_{3} r_{1}\right) j_{\ell}\left(k_{2} r_{2}\right) j_{\ell^{\prime}}\left(k_{3} r_{3}\right)
\end{aligned}
$$

The reduced bispectrum is then given by

$$
b_{\ell_{1} \ell_{2} \ell_{3}}^{v \delta^{\prime}}=\frac{4}{\pi^{2}} Z_{\ell_{2} \ell_{3}}^{v \delta^{\prime}}\left(z_{1}, z_{2}, z_{3}\right)+\text { perm.. }
$$

Also this contribution vanishes if all three redshifts coincide, due to the different parities of the spherical Bessel functions involved. Using the definition (3.11) and the transfer function (3.29) we can express the bispectrum as

$$
\begin{aligned}
b_{\ell_{1} \ell_{2} \ell_{3}}^{v \delta^{\prime}}= & c_{\ell_{2}}^{v \delta}\left(z_{1}, z_{2}\right) c_{\ell_{3}}^{\delta^{\prime} \delta}\left(z_{1}, z_{3}\right)+c_{\ell_{2}}^{\delta^{\prime} \delta}\left(z_{1}, z_{2}\right) c_{\ell_{3}}^{v \delta}\left(z_{1}, z_{3}\right) \\
& +c_{\ell_{1}}^{v \delta}\left(z_{2}, z_{1}\right) c_{\ell_{3}}^{\delta^{\prime} \delta}\left(z_{2}, z_{3}\right)+c_{\ell_{1}}^{\delta^{\prime} \delta}\left(z_{2}, z_{1}\right) c_{\ell_{3}}^{v \delta}\left(z_{2}, z_{3}\right) \\
& +c_{\ell_{1}}^{v \delta}\left(z_{3}, z_{1}\right) c_{\ell_{2}}^{\delta^{\prime} \delta}\left(z_{3}, z_{2}\right)+c_{\ell_{1}}^{\delta^{\prime} \delta}\left(z_{3}, z_{1}\right) c_{\ell_{2}}^{v \delta}\left(z_{3}, z_{2}\right) .
\end{aligned}
$$




\section{A.5 Term $\mathcal{H}^{-1} \partial_{r}^{2} v \delta$}

We do not compute this term here since this is done in Ref. [15] but we repeat the result for completeness.

$$
\begin{aligned}
b_{\ell_{1} \ell_{2} \ell_{3}}^{v^{\prime} \delta}= & c_{\ell_{2}}^{v^{\prime} \delta}\left(z_{1}, z_{2}\right) c_{\ell_{3}}^{\delta \delta}\left(z_{1}, z_{3}\right)+c_{\ell_{2}}^{\delta \delta}\left(z_{1}, z_{2}\right) c_{\ell_{3}}^{v^{\prime} \delta}\left(z_{1}, z_{3}\right) \\
& +c_{\ell_{1}}^{v^{\prime} \delta}\left(z_{2}, z_{1}\right) c_{\ell_{3}}^{\delta \delta}\left(z_{2}, z_{3}\right)+c_{\ell_{1}}^{\delta \delta}\left(z_{1}, z_{2}\right) c_{\ell_{3}}^{v^{\prime} \delta}\left(z_{2}, z_{3}\right) \\
& +c_{\ell_{1}}^{v^{\prime} \delta}\left(z_{3}, z_{1}\right) c_{\ell_{2}}^{\delta \delta}\left(z_{2}, z_{3}\right)+c_{\ell_{1}}^{\delta \delta}\left(z_{1}, z_{3}\right) c_{\ell_{2}}^{v^{\prime} \delta}\left(z_{3}, z_{2}\right)
\end{aligned}
$$

\section{A.6 Term $\mathcal{H}^{-1} \partial_{r}^{2} v \kappa$}

We compute the term

$$
\left\langle\left(-2 \mathcal{H}^{-1} \partial_{r}^{2} v \kappa\right)\left(\mathbf{n}_{1}, z_{1}\right) \delta\left(\mathbf{n}_{2}, z_{2}\right) \delta\left(\mathbf{n}_{3}, z_{3}\right)\right\rangle_{c}+\text { perm. . }
$$

Following the same approach of previous sections we obtain

$$
\begin{aligned}
\langle\ldots\rangle= & -\frac{1}{\mathcal{H}\left(z_{1}\right)(2 \pi)^{12}} \int d^{3} k d^{3} k_{1} d^{3} k_{2} d^{3} k_{3} \int_{0}^{r_{1}} d r^{\prime} \frac{r_{1}-r^{\prime}}{r_{1} r^{\prime}} k \partial_{\left(k r_{1}\right)}^{2} e^{i \mathbf{k} \cdot \mathbf{n}_{1} r_{1}} \\
& \times\left(\Delta_{2} e^{i \mathbf{k}_{1} \cdot \mathbf{n}_{1} r^{\prime}}\right) e^{i\left(\mathbf{k}_{2} \cdot \mathbf{n}_{2} r_{2}+\mathbf{k}_{3} \cdot \mathbf{n}_{3} r_{3}\right)} \\
& \times T_{v}\left(k, \eta_{1}\right) T_{\Phi+\Psi}\left(k_{1}, \eta^{\prime}\right) T_{\delta}\left(k_{2}, \eta_{2}\right) T_{\delta}\left(k_{3}, \eta_{3}\right)\left\langle R(\mathbf{k}) R\left(\mathbf{k}_{1}\right) R\left(\mathbf{k}_{2}\right) R\left(\mathbf{k}_{3}\right)\right\rangle \\
= & -\frac{1}{\mathcal{H}\left(z_{1}\right)(2 \pi)^{6}} \int d^{3} k_{2} d^{3} k_{3} \int_{0}^{r_{1}} d r^{\prime} \frac{r_{1}-r^{\prime}}{r_{1} r^{\prime}} k_{2} \partial_{\left(k_{2} r_{1}\right)}^{2} e^{-i \mathbf{k}_{2} \cdot \mathbf{n}_{1} r_{1}}\left(\Delta_{2} e^{-i \mathbf{k}_{3} \cdot \mathbf{n}_{1} r^{\prime}}\right) \\
& \times e^{i\left(\mathbf{k}_{2} \cdot \mathbf{n}_{2} r_{2}+\mathbf{k}_{3} \cdot \mathbf{n}_{3} r_{3}\right)} T_{v}\left(k_{2}, \eta_{1}\right) T_{\Phi+\Psi}\left(k_{3}, \eta^{\prime}\right) T_{\delta}\left(k_{2}, \eta_{2}\right) T_{\delta}\left(k_{3}, \eta_{3}\right) P_{R}\left(k_{2}\right) P_{R}\left(k_{3}\right) \\
& -\frac{1}{\mathcal{H}\left(z_{1}\right)(2 \pi)^{6}} \int d^{3} k_{2} d^{3} k_{3} \int_{0}^{r_{1}} d r^{\prime} \frac{r_{1}-r^{\prime}}{r_{1} r^{\prime}} k_{3} \partial_{\left(k_{3} r_{1}\right)}^{2} e^{-i \mathbf{k}_{3} \cdot \mathbf{n}_{1} r_{1}}\left(\Delta_{2} e^{-i \mathbf{k}_{2} \cdot \mathbf{n}_{1} r^{\prime}}\right) \\
& \times e^{i\left(\mathbf{k}_{2} \cdot \mathbf{n}_{2} r_{2}+\mathbf{k}_{3} \cdot \mathbf{n}_{3} r_{3}\right)} T_{v}\left(k_{3}, \eta_{1}\right) T_{\Phi+\Psi}\left(k_{2}, \eta^{\prime}\right) T_{\delta}\left(k_{2}, \eta_{2}\right) T_{\delta}\left(k_{3}, \eta_{3}\right) P_{R}\left(k_{2}\right) P_{R}\left(k_{3}\right)
\end{aligned}
$$

which leads to

$$
\begin{aligned}
B^{v^{\prime} \kappa}\left(\mathbf{n}_{1}, \mathbf{n}_{2}, \mathbf{n}_{3}, z_{1}, z_{2}, z_{3}\right)= & \frac{4}{\pi^{2}} \sum_{\substack{\ell, \ell^{\prime} \\
m, m^{\prime}}} Y_{\ell m}\left(\mathbf{n}_{1}\right) Y_{\ell^{\prime} m^{\prime}}\left(\mathbf{n}_{1}\right) Y_{\ell m}^{*}\left(\mathbf{n}_{2}\right) Y_{\ell^{\prime} m^{\prime}}^{*}\left(\mathbf{n}_{3}\right) Z_{\ell \ell^{\prime}}^{v^{\prime} \kappa}\left(z_{1}, z_{2}, z_{3}\right) \\
& + \text { perm. }
\end{aligned}
$$

where

$$
\begin{aligned}
& Z_{\ell \ell^{\prime}}^{v^{\prime}}\left(z_{1}, z_{2}, z_{3}\right) \\
& =\frac{1}{\mathcal{H}\left(z_{1}\right)} \int d k_{2} d k_{3} k_{2}^{3} k_{3}^{2} P_{R}\left(k_{2}\right) P_{R}\left(k_{3}\right) \int_{0}^{r_{1}} d r^{\prime} \frac{r_{1}-r^{\prime}}{r_{1} r^{\prime}} T_{v}\left(k_{2}, \eta_{1}\right) T_{\Psi+\Phi}\left(k_{3}, \eta^{\prime}\right) T_{\delta}\left(k_{2}, \eta_{2}\right) \\
& \times T_{\delta}\left(k_{3}, \eta_{3}\right) \ell^{\prime}\left(\ell^{\prime}+1\right) j_{\ell}^{\prime}\left(k_{2} r_{1}\right) j_{\ell^{\prime}}\left(k_{3} r^{\prime}\right) j_{\ell}\left(k_{2} r_{2}\right) j_{\ell^{\prime}}\left(k_{3} r_{3}\right) \\
& +\frac{1}{\mathcal{H}\left(z_{1}\right)} \int d k_{2} d k_{3} k_{2}^{2} k_{3}^{2} P_{R}\left(k_{2}\right) P_{R}\left(k_{3}\right) \int_{0}^{r_{1}} d r^{\prime} \frac{r_{1}-r^{\prime}}{r_{1} r^{\prime}} T_{v}\left(k_{3}, \eta_{1}\right) T_{\Psi+\Phi}\left(k_{2}, \eta^{\prime}\right) T_{\delta}\left(k_{2}, \eta_{2}\right) \\
& \times T_{\delta}\left(k_{3}, \eta_{3}\right) \ell(\ell+1) j_{\ell}\left(k_{2} r^{\prime}\right) j_{\ell^{\prime}}^{\prime}\left(k_{3} r_{1}\right) j_{\ell}\left(k_{2} r_{2}\right) j_{\ell^{\prime}}\left(k_{3} r_{3}\right) .
\end{aligned}
$$


Hence, the reduced bispectrum is

$$
b_{\ell_{1} \ell_{2} \ell_{3}}^{v^{\prime} \kappa}=\frac{4}{\pi^{2}} Z_{\ell_{2} \ell_{3}}^{v^{\prime} \kappa}\left(z_{1}, z_{2}, z_{3}\right)+\text { perm. }
$$

and it can be rewritten in terms of products of power spectra, using the transfer function in multipole space (3.30), as

$$
\begin{aligned}
b_{\ell_{1} \ell_{2} \ell_{3}}^{v^{\prime} \kappa}= & c_{\ell_{2}}^{v^{\prime} \delta}\left(z_{1}, z_{2}\right) c_{\ell_{3}}^{\kappa \delta}\left(z_{1}, z_{3}\right)+c_{\ell_{2}}^{\kappa \delta}\left(z_{1}, z_{2}\right) c_{\ell_{3}}^{v^{\prime} \delta}\left(z_{1}, z_{3}\right) \\
& +c_{\ell_{1}}^{v^{\prime} \delta}\left(z_{2}, z_{1}\right) c_{\ell_{3}}^{\kappa \delta}\left(z_{2}, z_{3}\right)+c_{\ell_{1}}^{\kappa \delta}\left(z_{2}, z_{1}\right) c_{\ell_{3}}^{v^{\prime} \delta}\left(z_{2}, z_{3}\right) \\
& +c_{\ell_{1}}^{v^{\prime} \delta}\left(z_{3}, z_{1}\right) c_{\ell_{2}}^{\kappa \delta}\left(z_{3}, z_{2}\right)+c_{\ell_{1}}^{\kappa \delta}\left(z_{3}, z_{1}\right) c_{\ell_{2}}^{v^{\prime} \delta}\left(z_{3}, z_{2}\right) .
\end{aligned}
$$

\section{A.7 Term $\kappa \delta$}

This term has been computed in detail in Ref. [15]. We repeat only the result here for completeness.

$$
\begin{aligned}
b_{\ell_{1} \ell_{2} \ell_{3}}^{\kappa \delta}= & c_{\ell_{2}}^{\kappa \delta}\left(z_{1}, z_{2}\right) c_{\ell_{3}}^{\delta \delta}\left(z_{1}, z_{3}\right)+c_{\ell_{3}}^{\kappa \delta}\left(z_{1}, z_{3}\right) c_{\ell_{2}}^{\delta \delta}\left(z_{1}, z_{2}\right) \\
& +c_{\ell_{1}}^{\kappa \delta}\left(z_{2}, z_{1}\right) c_{\ell_{3}}^{\delta \delta}\left(z_{2}, z_{3}\right)+c_{\ell_{3}}^{\kappa \delta}\left(z_{2}, z_{3}\right) c_{\ell_{1}}^{\delta \delta}\left(z_{1}, z_{2}\right) \\
& +c_{\ell_{1}}^{\kappa \delta}\left(z_{3}, z_{1}\right) c_{\ell_{2}}^{\delta \delta}\left(z_{2}, z_{3}\right)+c_{\ell_{2}}^{\kappa \delta}\left(z_{3}, z_{2}\right) c_{\ell_{1}}^{\delta \delta}\left(z_{1}, z_{3}\right) .
\end{aligned}
$$

\section{A.8 Term $\kappa^{2}$}

We consider

$$
\left\langle\left(2 \kappa^{2}\right)\left(\mathbf{n}_{1}, z_{1}\right) \delta\left(\mathbf{n}_{2}, z_{2}\right) \delta\left(\mathbf{n}_{3}, z_{3}\right)\right\rangle_{c}+\text { perm. },
$$

from which we compute

$$
\begin{aligned}
\langle\ldots\rangle= & \frac{1}{2(2 \pi)^{12}} \int d^{3} k d^{3} k_{1} d^{3} k_{2} d^{3} k_{3} \int_{0}^{r_{1}} d r \frac{r_{1}-r}{r_{1} r} \int_{0}^{r_{1}} d r^{\prime} \frac{r_{1}-r^{\prime}}{r_{1} r^{\prime}}\left(\Delta_{2} e^{i \mathbf{k} \cdot \mathbf{n}_{1} r}\right)\left(\Delta_{2} e^{i \mathbf{k}_{1} \cdot \mathbf{n}_{1} r^{\prime}}\right) \\
& \times e^{i\left(\mathbf{k}_{2} \cdot \mathbf{n}_{2} r_{2}+\mathbf{k}_{3} \cdot \mathbf{n}_{3} r_{3}\right)} T_{\Psi+\Phi}(k, \eta) T_{\Phi+\Psi}\left(k_{1}, \eta^{\prime}\right) \\
& \times T_{\delta}\left(k_{2}, \eta_{2}\right) T_{\delta}\left(k_{3}, \eta_{3}\right)\left\langle R(\mathbf{k}) R\left(\mathbf{k}_{1}\right) R\left(\mathbf{k}_{2}\right) R\left(\mathbf{k}_{3}\right)\right\rangle \\
= & \frac{1}{(2 \pi)^{6}} \int d^{3} k_{2} d^{3} k_{3} \int_{0}^{r_{1}} d r \frac{r_{1}-r}{r_{1} r} \int_{0}^{r_{1}} d r^{\prime} \frac{r_{1}-r^{\prime}}{r_{1} r^{\prime}}\left(\Delta_{2} e^{-i \mathbf{k}_{2} \cdot \mathbf{n}_{1} r}\right)\left(\Delta_{2} e^{-i \mathbf{k}_{3} \cdot \mathbf{n}_{1} r^{\prime}}\right) \\
& \times e^{i\left(\mathbf{k}_{2} \cdot \mathbf{n}_{2} r_{2}+\mathbf{k}_{3} \cdot \mathbf{n}_{3} r_{3}\right)} T_{\Psi+\Phi}\left(k_{2}, \eta\right) T_{\Phi+\Psi}\left(k_{3}, \eta^{\prime}\right) T_{\delta}\left(k_{2}, \eta_{2}\right) T_{\delta}\left(k_{3}, \eta_{3}\right) P_{R}\left(k_{2}\right) P_{R}\left(k_{3}\right) .
\end{aligned}
$$

Analogously to previous section, we get

$$
\begin{aligned}
B^{\kappa^{2}}\left(\mathbf{n}_{1}, \mathbf{n}_{2}, \mathbf{n}_{3}, z_{1}, z_{2}, z_{3}\right)= & \frac{4}{\pi^{2}} \sum_{\substack{\ell, \ell^{\prime} \\
m, m^{\prime}}} Y_{\ell m}\left(\mathbf{n}_{1}\right) Y_{\ell^{\prime} m^{\prime}}\left(\mathbf{n}_{1}\right) Y_{\ell m}^{*}\left(\mathbf{n}_{2}\right) Y_{\ell^{\prime} m^{\prime}}^{*}\left(\mathbf{n}_{3}\right) Z_{\ell \ell^{\prime}}^{\kappa^{2}}\left(z_{1}, z_{2}, z_{3}\right) \\
& + \text { perm. }
\end{aligned}
$$

where

$$
\begin{aligned}
Z_{\ell \ell^{\prime}}^{\kappa^{2}}\left(z_{1}, z_{2}, z_{3}\right)= & \int d k_{2} d k_{3} k_{2}^{2} k_{3}^{2} P_{R}\left(k_{2}\right) P_{R}\left(k_{3}\right) \int_{0}^{r_{1}} d r \frac{r_{1}-r}{r_{1} r} \int_{0}^{r_{1}} d r^{\prime} \frac{r_{1}-r^{\prime}}{r_{1} r^{\prime}} \\
& \times T_{\Psi+\Phi}\left(k_{2}, \eta\right) T_{\Psi+\Phi}\left(k_{3}, \eta^{\prime}\right) T_{\delta}\left(k_{2}, \eta_{2}\right) T_{\delta}\left(k_{3}, \eta_{3}\right) \\
& \times \ell(\ell+1) \ell^{\prime}\left(\ell^{\prime}+1\right) j_{\ell}\left(k_{2} r\right) j_{\ell^{\prime}}\left(k_{3} r^{\prime}\right) j_{\ell}\left(k_{2} r_{2}\right) j_{\ell^{\prime}}\left(k_{3} r_{3}\right)
\end{aligned}
$$


Therefore, the reduced bispectrum is

$$
b_{\ell_{1} \ell_{2} \ell_{3}}^{\kappa^{2}}=\frac{4}{\pi^{2}} Z_{\ell_{2} \ell_{3}}^{\kappa^{2}}\left(z_{1}, z_{2}, z_{3}\right)+\text { perm. }
$$

and in terms of products of power spectra it becomes

$$
b_{\ell_{1} \ell_{2} \ell_{3}}^{\kappa^{2}}=c_{\ell_{2}}^{\kappa \delta}\left(z_{1}, z_{2}\right) c_{\ell_{3}}^{\kappa \delta}\left(z_{1}, z_{3}\right)+c_{\ell_{1}}^{\kappa \delta}\left(z_{2}, z_{1}\right) c_{\ell_{3}}^{\kappa \delta}\left(z_{2}, z_{3}\right)+c_{\ell_{1}}^{\kappa \delta}\left(z_{3}, z_{1}\right) c_{\ell_{2}}^{\kappa \delta}\left(z_{3}, z_{2}\right) .
$$

We remark that in Eq. (2.13) only the two terms, $\mathcal{H}^{-2} \partial_{r}^{2} v \partial_{r}^{2} v$ and $2 \kappa^{2}$, enter as squares of first order perturbations, but with a different numerical pre-factor. Indeed in terms of the first order lensing, i.e. $-2 \kappa$, at second order we have $2 \kappa^{2}=(-2 \kappa)^{2} / 2$. For this reason Eq. (A.57) has no pre-factor 2.

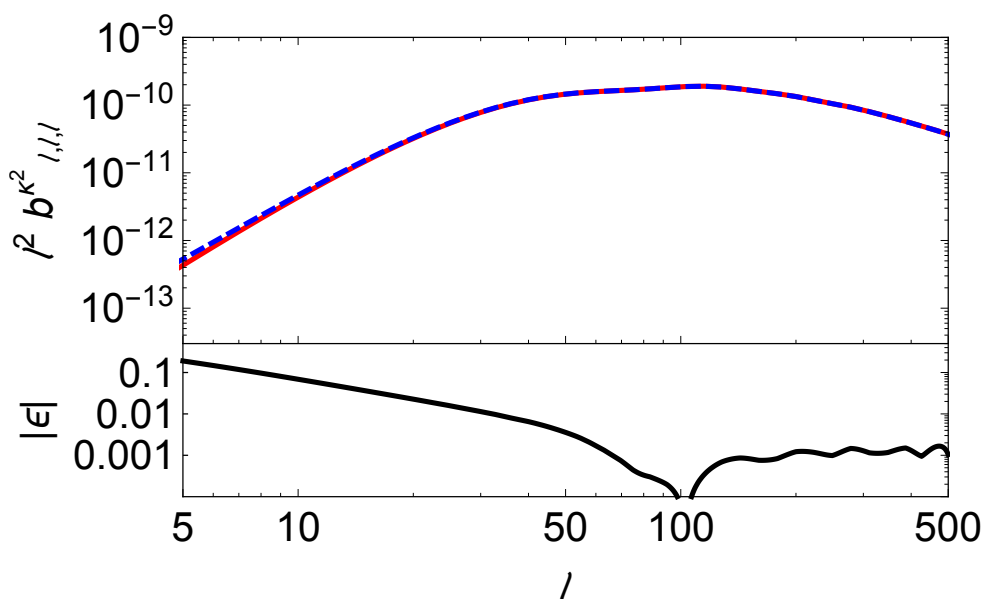

Figure 9. Limber approximation. The Limber approximation is excellent for all lensing like terms. Here we plot (top panel) the Limber approximation (solid red) and the full result (dashed blue) for the term $\kappa^{2}$ evaluated at the mean redshift $z=1$ and with a window function of width $\Delta z=1$, and the relative difference (bottom panel) $\epsilon=1-b_{\ell, \ell, \ell}^{\text {Limber }} / b_{\ell, \ell, \ell}^{\text {exact }}$.

In order to test the accuracy of the Limber approximation, that we use to evaluate the contribution of $b_{\ell_{1} \ell_{2} \ell_{3}}^{\int \nabla \kappa \Psi_{1}}$ and $b_{\ell_{1} \ell_{2} \ell_{3}}^{\int \Delta_{2}\left(\nabla \Psi_{1} \nabla \Psi_{1}\right)}$ to the total bispectrum with window functions (Fig. 8), we have checked it on the term $b_{\ell_{1} \ell_{2} \ell_{3}}^{\kappa^{2}}$. Our tests show that the Limber approximation for like-lensing terms in configurations with window functions is very accurate for $\ell \geq 20$. The difference between the fully integrated term and the Limber approximation is not visible by eye, see Fig. 9. Using Eq. (3.50) we easily obtain the following result for the Limber approximation of (A.55)

$$
\begin{aligned}
{\left[Z_{\ell \ell^{\prime}}^{\kappa^{2}}\left(z_{1}, z_{2}, z_{3}\right)\right]^{(\text {Limber })}=\Theta\left(z_{1}-z_{2}\right) \Theta\left(z_{1}-z_{3}\right) \frac{\pi^{2}}{4} \ell(\ell+1) \ell^{\prime}\left(\ell^{\prime}+1\right) \frac{1}{r_{2}^{2} r_{3}^{2}} \frac{r_{1}-r_{2}}{r_{1} r_{2}} \frac{r_{1}-r_{3}}{r_{1} r_{3}} } \\
\quad \times P_{R}\left(\frac{\ell+1 / 2}{r_{2}}\right) P_{R}\left(\frac{\ell^{\prime}+1 / 2}{r_{3}}\right) T_{\Psi+\Phi}\left(\frac{\ell+1 / 2}{r_{2}}, \eta_{2}\right) T_{\Psi+\Phi}\left(\frac{\ell^{\prime}+1 / 2}{r_{3}}, \eta_{3}\right) \\
\quad \times T_{\delta}\left(\frac{\ell+1 / 2}{r_{2}}, \eta_{2}\right) T_{\delta}\left(\frac{\ell^{\prime}+1 / 2}{r_{3}}, \eta_{3}\right) .
\end{aligned}
$$


It is interesting to note that here we have no Dirac- $\delta$ function, but two Heaviside- $\Theta$ functions due to the double integral along the line of sight. Therefore this term remains large even if all three redshifts are different, while in the $\kappa^{(2)}$-term in the Limber approximation, Eq. (3.57), at least two redshifts have to be equal for the result to remain substantial.

\section{A.9 Term $\nabla_{a} \delta \nabla^{a} \psi$}

Next we consider the lensing term,

$$
\left\langle\left(\nabla_{a} \delta \nabla^{a} \psi\right)\left(\mathbf{n}_{1}, z_{1}\right) \delta\left(\mathbf{n}_{2}, z_{2}\right) \delta\left(\mathbf{n}_{3}, z_{3}\right)\right\rangle_{c}+\text { perm. . }
$$

To profit from the known identities of the spin raising and spin lowering operators, see e.g. [51], we introduce the helicity basis $\left\{\mathbf{e}_{+}, \mathbf{e}_{-}\right\}$, where

$$
\mathbf{e}_{+}=\frac{\mathbf{e}_{1}-i \mathbf{e}_{2}}{\sqrt{2}}, \quad \mathbf{e}_{-}=\frac{\mathbf{e}_{1}+i \mathbf{e}_{2}}{\sqrt{2}}
$$

with $\left\{\mathbf{e}_{\mathbf{1}}, \mathbf{e}_{\mathbf{2}}\right\}=\left\{\partial_{\theta}, \frac{1}{\sin (\theta)} \partial_{\phi}\right\}$. In terms of the helicity basis the metric on the sphere is determined by $\gamma_{0}^{++}=\gamma_{0}^{--}=0$ and $\gamma_{0}^{+-}=\gamma_{0}^{-+}=1$ so that

$$
\left(\nabla_{a} \delta \nabla^{a} \psi\right)=\nabla_{+} \delta \nabla_{-} \psi+\nabla_{-} \delta \nabla_{+} \psi .
$$

Using Eq. (A4.69) in Ref. [51],

$$
\not \partial=-\sqrt{2} \nabla_{-} \quad \text { and } \quad \not \partial^{*}=-\sqrt{2} \nabla_{+},
$$

we can rewrite the previous expression in terms of lowering and rising spin operators,

$$
\left(\nabla_{a} \delta \nabla^{a} \psi\right)=\frac{1}{2} \not \partial^{*} \delta \not \partial \psi+\frac{1}{2} \not \partial \delta \not^{*} \psi .
$$

We then compute

$$
\begin{aligned}
\langle\cdots\rangle= & -\frac{1}{(2 \pi)^{12}} \int d k^{3} d k_{1}^{3} d k_{2}^{3} d k_{3}^{3} \int_{0}^{r_{1}} d r \frac{r_{1}-r}{r_{1} r} \\
& \times \frac{1}{2}\left(\not \partial^{*} e^{i \mathbf{k}_{1} \cdot \mathbf{n}_{1} r_{1}} \not \partial e^{i \mathbf{k} \cdot \mathbf{n}_{1} r}+\not \partial e^{i \mathbf{k}_{1} \cdot \mathbf{n}_{1} r_{1}} \not \partial^{*} e^{i \mathbf{k} \cdot \mathbf{n}_{1} r}\right) e^{i\left(\mathbf{k}_{2} \cdot \mathbf{n}_{2} r_{2}+\mathbf{k}_{3} \cdot \mathbf{n}_{3} r_{3}\right)} \\
& \times T_{\Psi+\Phi}(k, \eta) T_{\delta}\left(k_{1}, \eta_{1}\right) T_{\delta}\left(k_{2}, \eta_{2}\right) T_{\delta}\left(k_{3}, \eta_{3}\right)\left\langle R(\mathbf{k}) R\left(\mathbf{k}_{1}\right) R\left(\mathbf{k}_{2}\right) R\left(\mathbf{k}_{3}\right)\right\rangle \\
= & -\frac{1}{(2 \pi)^{6}} \frac{1}{2} \int d k_{2}^{3} d k_{3}^{3} \int_{0}^{r_{1}} d r \frac{r_{1}-r}{r_{1} r} \\
& \times\left(\not \partial^{*} e^{-i \mathbf{k}_{3} \cdot \mathbf{n}_{1} r_{1}} \not \partial e^{-i \mathbf{k}_{2} \cdot \mathbf{n}_{1} r}+\not \partial e^{-i \mathbf{k}_{3} \cdot \mathbf{n}_{1} r_{1}} \not \not^{*} e^{-i \mathbf{k}_{2} \cdot \mathbf{n}_{1} r}\right) e^{i\left(\mathbf{k}_{2} \cdot \mathbf{n}_{2} r_{2}+\mathbf{k}_{3} \cdot \mathbf{n}_{3} r_{3}\right)} \\
& \times T_{\Psi+\Phi}\left(k_{2}, \eta\right) T_{\delta}\left(k_{3}, \eta_{1}\right) T_{\delta}\left(k_{2}, \eta_{2}\right) T_{\delta}\left(k_{3}, \eta_{3}\right) P\left(k_{2}\right) P_{R}\left(k_{3}\right) \\
& -\frac{1}{(2 \pi)^{6}} \frac{1}{2} \int d k_{2}^{3} d k_{3}^{3} \int_{0}^{r_{1}} d r \frac{r_{1}-r}{r_{1} r} \\
& \times\left(\not \partial^{*} e^{-i \mathbf{k}_{2} \cdot \mathbf{n}_{1} r_{1}} \not \partial e^{-i \mathbf{k}_{3} \cdot \mathbf{n}_{1} r}+\not \partial e^{-i \mathbf{k}_{2} \cdot \mathbf{n}_{1} r_{1}} \not \not^{*} e^{-i \mathbf{k}_{3} \cdot \mathbf{n}_{1} r}\right) e^{i\left(\mathbf{k}_{2} \cdot \mathbf{n}_{2} r_{2}+\mathbf{k}_{3} \cdot \mathbf{n}_{3} r_{3}\right)} \\
& \times T_{\Psi+\Phi}\left(k_{3}, \eta\right) T_{\delta}\left(k_{2}, \eta_{1}\right) T_{\delta}\left(k_{2}, \eta_{2}\right) T_{\delta}\left(k_{3}, \eta_{3}\right) P_{R}\left(k_{2}\right) P_{R}\left(k_{3}\right) .
\end{aligned}
$$

We now use the identities

$$
\begin{aligned}
\not{ }_{s} Y_{\ell m} & =\sqrt{(\ell-s)(\ell+s+1)}{ }_{s+1} Y_{\ell m}, \\
\not^{*}{ }_{s} Y_{\ell m} & =-\sqrt{(\ell+s)(\ell-s+1)}{ }_{s-1} Y_{\ell m},
\end{aligned}
$$


which lead to

$$
\begin{aligned}
\not \partial e^{i \mathbf{k} \cdot \mathbf{n} r} & =4 \pi \sum_{\ell m} i^{\ell} j_{\ell}(k r) \sqrt{\ell(\ell+1)}{ }_{1} Y_{\ell m}(\mathbf{n}) Y_{\ell m}^{*}(\hat{\mathbf{k}}), \\
\not{ }^{*} e^{i \mathbf{k} \cdot \mathbf{n} r} & =-4 \pi \sum_{\ell m} i^{\ell} j_{\ell}(k r) \sqrt{\ell(\ell+1)}{ }_{-1} Y_{\ell m}(\mathbf{n}) Y_{\ell m}^{*}(\hat{\mathbf{k}}) .
\end{aligned}
$$

Inserting these identities in (A.64) we find the bispectrum

$$
\begin{aligned}
B^{\nabla \delta \nabla \psi}\left(\mathbf{n}_{1}, \mathbf{n}_{2}, \mathbf{n}_{3}, z_{1}, z_{2}, z_{3}\right)= & \frac{4}{\pi^{2}} \sum_{\substack{\ell, \ell^{\prime} \\
m, m^{\prime}}} \frac{1}{2}\left[{ }_{-1} Y_{\ell m}\left(\mathbf{n}_{1}\right)_{1} Y_{\ell^{\prime} m^{\prime}}\left(\mathbf{n}_{1}\right)+{ }_{1} Y_{\ell m}\left(\mathbf{n}_{1}\right)_{-1} Y_{\ell^{\prime} m^{\prime}}\left(\mathbf{n}_{1}\right)\right] \\
& \times Y_{\ell m}^{*}\left(\mathbf{n}_{2}\right) Y_{\ell^{\prime} m^{\prime}}^{*}\left(\mathbf{n}_{3}\right) Z_{\ell \ell^{\prime}}^{\nabla \delta \psi}\left(z_{1}, z_{2}, z_{3}\right)+\text { perm. }, \quad
\end{aligned}
$$

where

$$
\begin{aligned}
& Z_{\ell \ell^{\prime}}^{\nabla \delta \psi}\left(z_{1}, z_{2}, z_{3}\right) \\
&=\int d k_{2} k_{2}^{2} d k_{3} k_{3}^{2} \int_{0}^{r_{1}} d r \frac{r_{1}-r}{r_{1} r} \sqrt{\ell(\ell+1)} \sqrt{\ell^{\prime}\left(\ell^{\prime}+1\right)} j_{\ell}\left(k_{2} r\right) j_{\ell^{\prime}}\left(k_{3} r_{1}\right) j_{\ell}\left(k_{2} r_{2}\right) j_{\ell^{\prime}}\left(k_{3} r_{3}\right) \\
& \quad \times T_{\Psi+\Phi}\left(k_{2}, \eta\right) T_{\delta}\left(k_{3}, \eta_{1}\right) T_{\delta}\left(k_{2}, \eta_{2}\right) T_{\delta}\left(k_{3}, \eta_{3}\right) P_{R}\left(k_{2}\right) P_{R}\left(k_{3}\right) \\
&+\int d k_{2} k_{2}^{2} d k_{3} k_{3}^{2} \int_{0}^{r_{1}} d r \frac{r_{1}-r}{r_{1} r} \sqrt{\ell(\ell+1)} \sqrt{\ell^{\prime}\left(\ell^{\prime}+1\right)} j_{\ell^{\prime}}\left(k_{3} r\right) j_{\ell}\left(k_{2} r_{1}\right) j_{\ell}\left(k_{2} r_{2}\right) j_{\ell^{\prime}}\left(k_{3} r_{3}\right) \\
& \times T_{\Psi+\Phi}\left(k_{3}, \eta\right) T_{\delta}\left(k_{2}, \eta_{1}\right) T_{\delta}\left(k_{2}, \eta_{2}\right) T_{\delta}\left(k_{3}, \eta_{3}\right) P_{R}\left(k_{2}\right) P_{R}\left(k_{3}\right) .
\end{aligned}
$$

We now introduce the generalized Gaunt integral

$$
\begin{aligned}
& \int d \Omega s_{s_{1}} Y_{\ell_{1} m_{1}}(\mathbf{n}) s_{2} Y_{\ell_{2} m_{2}}(\mathbf{n}){ }_{s_{3}} Y_{\ell_{3} m_{3}}(\mathbf{n}) \\
&=\sqrt{\frac{\left(2 \ell_{1}+1\right)\left(2 \ell_{2}+1\right)\left(2 \ell_{3}+1\right)}{4 \pi}}\left(\begin{array}{ccc}
\ell_{1} & \ell_{2} & \ell_{3} \\
-s_{1} & -s_{2} & -s_{3}
\end{array}\right)\left(\begin{array}{ccc}
\ell_{1} & \ell_{2} & \ell_{3} \\
m_{1} & m_{2} & m_{3}
\end{array}\right),
\end{aligned}
$$

and we define

$$
A_{\ell_{1} \ell_{2} \ell_{3}} \equiv \frac{1}{2} \frac{\left[\left(\begin{array}{ccc}
\ell_{1} & \ell_{2} & \ell_{3} \\
0 & 1 & -1
\end{array}\right)+\left(\begin{array}{ccc}
\ell_{1} & \ell_{2} & \ell_{3} \\
0 & -1 & 1
\end{array}\right)\right]}{\left(\begin{array}{ccc}
\ell_{1} & \ell_{2} & \ell_{3} \\
0 & 0 & 0
\end{array}\right)} .
$$

With this we can express the reduced bispectrum as

$$
b_{\ell_{1} \ell_{2} \ell_{3}}^{\nabla \delta \nabla \psi}=\frac{4}{\pi^{2}} A_{\ell_{1} \ell_{2} \ell_{3}} Z_{\ell_{2} \ell_{3}}^{\nabla \delta \nabla \psi}\left(z_{1}, z_{2}, z_{3}\right)+\text { perm. . }
$$

This can also be written in terms of the following angular power spectra

$$
\begin{aligned}
b_{\ell_{1} \ell_{2} \ell_{3}}^{\nabla \delta \nabla \psi}= & A_{\ell_{1} \ell_{2} \ell_{3}}\left(\sqrt{\frac{\ell_{3}\left(\ell_{3}+1\right)}{\ell_{2}\left(\ell_{2}+1\right)}} c_{\ell_{2}}^{\kappa \delta}\left(z_{1}, z_{2}\right) c_{\ell_{3}}^{\delta \delta}\left(z_{1}, z_{3}\right)+\sqrt{\frac{\ell_{2}\left(\ell_{2}+1\right)}{\ell_{3}\left(\ell_{3}+1\right)}} c_{\ell_{3}}^{\kappa \delta}\left(z_{1}, z_{3}\right) c_{\ell_{2}}^{\delta \delta}\left(z_{1}, z_{2}\right)\right) \\
& +A_{\ell_{2} \ell_{1} \ell_{3}}\left(\sqrt{\frac{\ell_{3}\left(\ell_{3}+1\right)}{\ell_{1}\left(\ell_{1}+1\right)}} c_{\ell_{1}}^{\kappa \delta}\left(z_{2}, z_{1}\right) c_{\ell_{3}}^{\delta \delta}\left(z_{2}, z_{3}\right)+\sqrt{\frac{\ell_{1}\left(\ell_{1}+1\right)}{\ell_{3}\left(\ell_{3}+1\right)}} c_{\ell_{3}}^{\kappa \delta}\left(z_{2}, z_{3}\right) c_{\ell_{1}}^{\delta \delta}\left(z_{2}, z_{1}\right)\right) \\
& +A_{\ell_{3} \ell_{1} \ell_{2}}\left(\sqrt{\frac{\ell_{2}\left(\ell_{2}+1\right)}{\ell_{1}\left(\ell_{1}+1\right)}} c_{\ell_{1}}^{\kappa \delta}\left(z_{3}, z_{1}\right) c_{\ell_{2}}^{\delta \delta}\left(z_{3}, z_{2}\right)+\sqrt{\frac{\ell_{1}\left(\ell_{1}+1\right)}{\ell_{2}\left(\ell_{2}+1\right)}} c_{\ell_{2}}^{\kappa \delta}\left(z_{3}, z_{2}\right) c_{\ell_{1}}^{\delta \delta}\left(z_{3}, z_{1}\right)\right) .
\end{aligned}
$$




\section{A.10 Term $\mathcal{H}^{-1} \nabla_{a} \partial_{r}^{2} v \nabla^{a} \psi$}

We consider

$$
\left\langle\left(\mathcal{H}^{-1} \nabla_{a} \partial_{r}^{2} v \nabla^{a} \psi\right)\left(\mathbf{n}_{1}, z_{1}\right) \delta\left(\mathbf{n}_{2}, z_{2}\right) \delta\left(\mathbf{n}_{3}, z_{3}\right)\right\rangle_{c}+\text { perm. . }
$$

Analogously to the previous case we have

$$
\mathcal{H}^{-1} \nabla_{a} \partial_{r}^{2} v \nabla^{a} \psi=\frac{1}{2} \not \partial^{*} \partial_{r}^{2} v \not \partial \psi+\frac{1}{2} \not \partial \partial_{r}^{2} v \not \partial^{*} \psi
$$

Let us compute (A.75),

$$
\begin{aligned}
\langle\cdots\rangle= & -\frac{1}{(2 \pi)^{12}} \frac{1}{\mathcal{H}\left(z_{1}\right)} \int d k^{3} d k_{1}^{3} d k_{2}^{3} d k_{3}^{3} k_{1} \int_{0}^{r_{1}} d r \frac{r_{1}-r}{r_{1} r} \\
& \times \frac{1}{2}\left(\not \not^{*} \partial_{\left(k_{1} r_{1}\right)}^{2} e^{i \mathbf{k}_{1} \cdot \mathbf{n}_{1} r_{1}} \not \partial e^{i \mathbf{k} \cdot \mathbf{n}_{1} r}+\not \partial_{\left(k_{1} r_{1}\right)}^{2} e^{i \mathbf{k}_{1} \cdot \mathbf{n}_{1} r_{1}} \not \partial^{*} e^{i \mathbf{k} \cdot \mathbf{n}_{1} r}\right) e^{i\left(\mathbf{k}_{2} \cdot \mathbf{n}_{2} r_{2}+\mathbf{k}_{3} \cdot \mathbf{n}_{3} r_{3}\right)} \\
& \times T_{\Psi+\Phi}(k, \eta) T_{v}\left(k_{1}, \eta_{1}\right) T_{\delta}\left(k_{2}, \eta_{2}\right) T_{\delta}\left(k_{3}, \eta_{3}\right)\left\langle R(\mathbf{k}) R\left(\mathbf{k}_{1}\right) R\left(\mathbf{k}_{2}\right) R\left(\mathbf{k}_{3}\right)\right\rangle \\
= & -\frac{1}{(2 \pi)^{6}} \frac{1}{2 \mathcal{H}\left(z_{1}\right)} \int d k_{2}^{3} d k_{3}^{3} k_{3} \int_{0}^{r_{1}} d r \frac{r_{1}-r}{r_{1} r} \\
& \times\left(\partial^{*} \partial_{\left(k_{3} r_{1}\right)}^{2} e^{-i \mathbf{k}_{3} \cdot \mathbf{n}_{1} r_{1}} \not \partial e^{-i \mathbf{k}_{2} \cdot \mathbf{n}_{1} r}+\not \partial \partial_{\left(k_{3} r_{1}\right)}^{2} e^{-i \mathbf{k}_{3} \cdot \mathbf{n}_{1} r_{1}} \not \partial^{*} e^{-i \mathbf{k}_{2} \cdot \mathbf{n}_{1} r}\right) e^{i\left(\mathbf{k}_{2} \cdot \mathbf{n}_{2} r_{2}+\mathbf{k}_{3} \cdot \mathbf{n}_{3} r_{3}\right)} \\
& \times T_{\Psi+\Phi}\left(k_{2}, \eta\right) T_{\delta}\left(k_{3}, \eta_{1}\right) T_{\delta}\left(k_{2}, \eta_{2}\right) T_{\delta}\left(k_{3}, \eta_{3}\right) P\left(k_{2}\right) P_{R}\left(k_{3}\right) \\
& -\frac{1}{(2 \pi)^{6}} \frac{1}{2 \mathcal{H}\left(z_{1}\right)} \int d k_{2}^{3} d k_{3}^{3} k_{2} \int_{0}^{r_{1}} d r \frac{r_{1}-r}{r_{1} r} \\
& \times\left(\not \partial^{*} \partial_{\left(k_{2} r_{1}\right)}^{2} e^{-i \mathbf{k}_{2} \cdot \mathbf{n}_{1} r_{1}} \not \partial e^{-i \mathbf{k}_{3} \cdot \mathbf{n}_{1} r}+\not \partial \partial_{\left(k_{2} r_{1}\right)}^{2} e^{-i \mathbf{k}_{2} \cdot \mathbf{n}_{1} r_{1}} \not \partial^{*} e^{-i \mathbf{k}_{3} \cdot \mathbf{n}_{1} r}\right) e^{i\left(\mathbf{k}_{2} \cdot \mathbf{n}_{2} r_{2}+\mathbf{k}_{3} \cdot \mathbf{n}_{3} r_{3}\right)} \\
& \times T_{\Psi+\Phi}\left(k_{3}, \eta\right) T_{\delta}\left(k_{2}, \eta_{1}\right) T_{\delta}\left(k_{2}, \eta_{2}\right) T_{\delta}\left(k_{3}, \eta_{3}\right) P_{R}\left(k_{2}\right) P_{R}\left(k_{3}\right) .
\end{aligned}
$$

Again, $\eta=\eta_{0}-r, \eta_{1}=\eta_{0}-r_{1}=\eta\left(z_{1}\right)$ etc. With this, the bispectrum is given by

$$
\begin{aligned}
B^{\nabla v^{\prime} \nabla \psi}\left(\mathbf{n}_{1}, \mathbf{n}_{2}, \mathbf{n}_{3}, z_{1}, z_{2}, z_{3}\right) & \\
= & \frac{4}{\pi^{2}} \sum_{\substack{\ell, \ell^{\prime} \\
m, m^{\prime}}} \frac{1}{2}\left[{ }_{-1} Y_{\ell m}\left(\mathbf{n}_{1}\right)_{1} Y_{\ell^{\prime} m^{\prime}}\left(\mathbf{n}_{1}\right)+{ }_{1} Y_{\ell m}\left(\mathbf{n}_{1}\right)_{-1} Y_{\ell^{\prime} m^{\prime}}\left(\mathbf{n}_{1}\right)\right] \\
& \times Y_{\ell m}^{*}\left(\mathbf{n}_{2}\right) Y_{\ell^{\prime} m^{\prime}}^{*}\left(\mathbf{n}_{3}\right) Z_{\ell \ell^{\prime}}^{\nabla v^{\prime} \nabla \psi}\left(z_{1}, z_{2}, z_{3}\right)+\text { perm. },
\end{aligned}
$$

where

$$
\begin{aligned}
Z_{\ell \ell^{\prime}}^{\nabla v^{\prime} \nabla \psi}\left(z_{1}, z_{2}, z_{3}\right)= & \frac{1}{\mathcal{H}\left(z_{1}\right)} \int d k_{2} k_{2}^{2} d k_{3} k_{3}^{3} \int_{0}^{r_{1}} d r \frac{r_{1}-r}{r_{1} r} \\
& \times T_{\Psi+\Phi}\left(k_{2}, \eta\right) T_{v}\left(k_{3}, \eta_{1}\right) T_{\delta}\left(k_{2}, \eta_{2}\right) T_{\delta}\left(k_{3}, \eta_{3}\right) P_{R}\left(k_{2}\right) P_{R}\left(k_{3}\right) \\
& \times j_{\ell}\left(k_{2} r\right) j_{\ell^{\prime}}^{\prime \prime}\left(k_{3} r_{1}\right) j_{\ell}\left(k_{2} r_{2}\right) j_{\ell^{\prime}}\left(k_{3} r_{3}\right) \sqrt{\ell(\ell+1)} \sqrt{\ell^{\prime}\left(\ell^{\prime}+1\right)} \\
& +\frac{1}{\mathcal{H}\left(z_{1}\right)} \int d k_{2} k_{2}^{3} d k_{3} k_{3}^{2} \int_{0}^{r_{1}} d r \frac{r_{1}-r}{r_{1} r} \\
& \times T_{\Psi+\Phi}\left(k_{3}, \eta\right) T_{v}\left(k_{2}, \eta_{1}\right) T_{\delta}\left(k_{2}, \eta_{2}\right) T_{\delta}\left(k_{3}, \eta_{3}\right) P_{R}\left(k_{2}\right) P_{R}\left(k_{3}\right) \\
& \times j_{\ell^{\prime}}\left(k_{3} r\right) j_{\ell}^{\prime \prime}\left(k_{2} r_{1}\right) j_{\ell}\left(k_{2} r_{2}\right) j_{\ell^{\prime}}\left(k_{3} r_{3}\right) \sqrt{\ell(\ell+1)} \sqrt{\ell^{\prime}\left(\ell^{\prime}+1\right)} .
\end{aligned}
$$

Like in the previous case, with the use of the generalised Gaunt factors, the reduced bispectrum is then given by

$$
b_{\ell_{1} \ell_{2} \ell_{3}}^{\nabla v^{\prime} \nabla \psi}=\frac{4}{\pi^{2}} A_{\ell_{1} \ell_{2} \ell_{3}} Z_{\ell_{2} \ell_{3}}^{\nabla v^{\prime} \nabla \psi}\left(z_{1}, z_{2}, z_{3}\right)+\text { perm. . }
$$


In terms of the angular power spectra this can be rewritten as

$$
\begin{aligned}
b_{\ell_{1} \ell_{2} \ell_{3}}^{\nabla v^{\prime} \nabla \psi}= & A_{\ell_{1} \ell_{2} \ell_{3}}\left(\sqrt{\frac{\ell_{3}\left(\ell_{3}+1\right)}{\ell_{2}\left(\ell_{2}+1\right)}} c_{\ell_{2}}^{\kappa \delta}\left(z_{1}, z_{2}\right) c_{\ell_{3}}^{v^{\prime} \delta}\left(z_{1}, z_{3}\right)+\sqrt{\frac{\ell_{2}\left(\ell_{2}+1\right)}{\ell_{3}\left(\ell_{3}+1\right)}} c_{\ell_{3}}^{\kappa \delta}\left(z_{1}, z_{3}\right) c_{\ell_{2}}^{v^{\prime} \delta}\left(z_{1}, z_{2}\right)\right) \\
& +A_{\ell_{2} \ell_{1} \ell_{3}}\left(\sqrt{\frac{\ell_{3}\left(\ell_{3}+1\right)}{\ell_{1}\left(\ell_{1}+1\right)}} c_{\ell_{1}}^{\kappa \delta}\left(z_{2}, z_{1}\right) c_{\ell_{3}}^{v^{\prime} \delta}\left(z_{2}, z_{3}\right)+\sqrt{\frac{\ell_{1}\left(\ell_{1}+1\right)}{\ell_{3}\left(\ell_{3}+1\right)}} c_{\ell_{3}}^{\kappa \delta}\left(z_{2}, z_{3}\right) c_{\ell_{1}}^{v^{\prime} \delta}\left(z_{2}, z_{1}\right)\right) \\
& +A_{\ell_{3} \ell_{1} \ell_{2}}\left(\sqrt{\frac{\ell_{2}\left(\ell_{2}+1\right)}{\ell_{1}\left(\ell_{1}+1\right)}} c_{\ell_{1}}^{\kappa \delta}\left(z_{3}, z_{1}\right) c_{\ell_{2}}^{v^{\prime} \delta}\left(z_{3}, z_{2}\right)+\sqrt{\frac{\ell_{1}\left(\ell_{1}+1\right)}{\ell_{2}\left(\ell_{2}+1\right)}} c_{\ell_{2}}^{\kappa \delta}\left(z_{3}, z_{2}\right) c_{\ell_{1}}^{v^{\prime} \delta}\left(z_{3}, z_{1}\right)\right) .
\end{aligned}
$$

\section{A.11 Term $\nabla_{b} \kappa \nabla^{b} \psi$}

We consider

$$
\left\langle\left(-2 \nabla_{b} \kappa \nabla^{b} \psi\right)\left(\mathbf{n}_{1}, z_{1}\right) \delta\left(\mathbf{n}_{2}, z_{2}\right) \delta\left(\mathbf{n}_{3}, z_{3}\right)\right\rangle_{c}+\text { perm. . }
$$

We start by rewriting

$$
-2 \nabla_{b} \kappa \nabla^{b} \psi=\left(\nabla_{b} \Delta_{2} \psi\right) \nabla^{b} \psi=\frac{1}{2} \not \partial^{*} \Delta_{2} \psi \not \partial \psi+\frac{1}{2} \not \partial \Delta_{2} \psi \not \partial^{*} \psi .
$$

We now compute (A.82)

$$
\begin{aligned}
\langle\cdots\rangle= & \frac{1}{(2 \pi)^{12}} \int d k^{3} d k_{1}^{3} d k_{2}^{3} d k_{3}^{3} \int_{0}^{r_{1}} d r \frac{r_{1}-r}{r_{1} r} \int_{0}^{r_{1}} d r^{\prime} \frac{r_{1}-r^{\prime}}{r_{1} r^{\prime}} \\
& \times \frac{1}{2}\left(\not \partial^{*} \Delta_{2} e^{i \mathbf{k} \cdot \mathbf{n}_{1} r} \not \partial e^{i \mathbf{k}_{1} \cdot \mathbf{n}_{1} r^{\prime}}+\not \partial \Delta_{2} e^{i \mathbf{k} \cdot \mathbf{n}_{1} r} \not \partial^{*} e^{i \mathbf{k}_{1} \cdot \mathbf{n}_{1} r^{\prime}}\right) e^{i\left(\mathbf{k}_{2} \cdot \mathbf{n}_{2} r_{2}+\mathbf{k}_{3} \cdot \mathbf{n}_{3} r_{3}\right)} \\
& \times T_{\Psi+\Phi}(k, \eta) T_{\Psi+\Phi}\left(k_{1}, \eta^{\prime}\right) T_{\delta}\left(k_{2}, \eta_{2}\right) T_{\delta}\left(k_{3}, \eta_{3}\right)\left\langle R(\mathbf{k}) R\left(\mathbf{k}_{1}\right) R\left(\mathbf{k}_{2}\right) R\left(\mathbf{k}_{3}\right)\right\rangle \\
= & \frac{1}{(2 \pi)^{6}} \int d k_{2}^{3} d k_{3}^{3} \int_{0}^{r_{1}} d r \frac{r_{1}-r}{r_{1} r} \int_{0}^{r_{1}} d r^{\prime} \frac{r_{1}-r^{\prime}}{r_{1} r^{\prime}} \\
& \times \frac{1}{2}\left(\not \partial^{*} \Delta_{2} e^{-i \mathbf{k}_{2} \cdot \mathbf{n}_{1} r} \not \partial e^{-i \mathbf{k}_{3} \cdot \mathbf{n}_{1} r^{\prime}}+\not \Delta_{2} e^{-i \mathbf{k}_{2} \cdot \mathbf{n}_{1} r} \not \not^{*} e^{-i \mathbf{k}_{3} \cdot \mathbf{n}_{1} r^{\prime}}\right) e^{i\left(\mathbf{k}_{2} \cdot \mathbf{n}_{2} r_{2}+\mathbf{k}_{3} \cdot \mathbf{n}_{3} r_{3}\right)} \\
& \times T_{\Psi+\Phi}\left(k_{2}, \eta\right) T_{\Psi+\Phi}\left(k_{3}, \eta^{\prime}\right) T_{\delta}\left(k_{2}, \eta_{2}\right) T_{\delta}\left(k_{3}, \eta_{3}\right) P_{R}\left(k_{2}\right) P_{R}\left(k_{3}\right) \\
& +\frac{1}{(2 \pi)^{6}} \int d k_{2}^{3} d k_{3}^{3} \int_{0}^{r_{1}} d r \frac{r_{1}-r}{r_{1} r} \int_{0}^{r_{1}} d r^{\prime} \frac{r_{1}-r^{\prime}}{r_{1} r^{\prime}} \\
& \times \frac{1}{2}\left(\not \partial^{*} \Delta_{2} e^{-i \mathbf{k}_{3} \cdot \mathbf{n}_{1} r} \not \partial e^{-i \mathbf{k}_{2} \cdot \mathbf{n}_{1} r^{\prime}}+\not \partial \Delta_{2} e^{-i \mathbf{k}_{3} \cdot \mathbf{n}_{1} r} \not \not^{*} e^{-i \mathbf{k}_{2} \cdot \mathbf{n}_{1} r^{\prime}}\right) e^{i\left(\mathbf{k}_{2} \cdot \mathbf{n}_{2} r_{2}+\mathbf{k}_{3} \cdot \mathbf{n}_{3} r_{3}\right)} \\
& \times T_{\Psi+\Phi}\left(k_{3}, \eta\right) T_{\Psi+\Phi}\left(k_{2}, \eta^{\prime}\right) T_{\delta}\left(k_{2}, \eta_{2}\right) T_{\delta}\left(k_{3}, \eta_{3}\right) P_{R}\left(k_{2}\right) P_{R}\left(k_{3}\right) .
\end{aligned}
$$

With this the bispectrum becomes

$$
\begin{aligned}
B^{\nabla_{b} \kappa \nabla^{b} \psi} & \left(\mathbf{n}_{1}, \mathbf{n}_{2}, \mathbf{n}_{3}, z_{1}, z_{2}, z_{3}\right) \\
= & \frac{4}{\pi^{2}} \sum_{\substack{\ell, \ell^{\prime} \\
m, m^{\prime}}} \frac{1}{2}\left[{ }_{1} Y_{\ell m}\left(\mathbf{n}_{1}\right)_{1} Y_{\ell^{\prime} m^{\prime}}\left(\mathbf{n}_{1}\right)+{ }_{1} Y_{\ell m}\left(\mathbf{n}_{1}\right)_{-1} Y_{\ell^{\prime} m^{\prime}}\left(\mathbf{n}_{1}\right)\right] Y_{\ell m}^{*}\left(\mathbf{n}_{2}\right) Y_{\ell^{\prime} m^{\prime}}^{*}\left(\mathbf{n}_{3}\right) \\
& \times Z_{\ell \ell^{\prime}}^{\nabla_{b} \kappa \nabla^{b} \psi}\left(z_{1}, z_{2}, z_{3}\right)+\text { perm. }
\end{aligned}
$$


where

$$
\begin{aligned}
Z_{\ell \ell^{\prime}}^{\nabla_{b} \kappa \nabla^{b} \psi}\left(z_{1}, z_{2}, z_{3}\right)= & \int d k_{2} k_{2}^{2} d k_{3} k_{3}^{3} \int_{0}^{r_{1}} d r \frac{r_{1}-r}{r_{1} r} \int_{0}^{r_{1}} d r^{\prime} \frac{r_{1}-r^{\prime}}{r_{1} r^{\prime}} \\
& \times T_{\Psi+\Phi}\left(k_{2}, \eta\right) T_{\Psi+\Phi}\left(k_{3}, \eta^{\prime}\right) T_{\delta}\left(k_{2}, \eta_{2}\right) T_{\delta}\left(k_{3}, \eta_{3}\right) P_{R}\left(k_{2}\right) P_{R}\left(k_{3}\right) \\
& \times j_{\ell}\left(k_{2} r\right) j_{\ell^{\prime}}\left(k_{3} r^{\prime}\right) j_{\ell}\left(k_{2} r_{2}\right) j_{\ell^{\prime}}\left(k_{3} r_{3}\right)(\ell(\ell+1))^{3 / 2} \sqrt{\ell^{\prime}\left(\ell^{\prime}+1\right)} \\
& +\int d k_{2} k_{2}^{3} d k_{3} k_{3}^{2} \int_{0}^{r_{1}} d r \frac{r_{1}-r}{r_{1} r} \int_{0}^{r_{1}} d r^{\prime} \frac{r_{1}-r^{\prime}}{r_{1} r^{\prime}} \\
& \times T_{\Psi+\Phi}\left(k_{3}, \eta\right) T_{\Psi+\Phi}\left(k_{2}, \eta^{\prime}\right) T_{\delta}\left(k_{2}, \eta_{2}\right) T_{\delta}\left(k_{3}, \eta_{3}\right) P_{R}\left(k_{2}\right) P_{R}\left(k_{3}\right) \\
& \times j_{\ell^{\prime}}\left(k_{3} r\right) j_{\ell}\left(k_{2} r^{\prime}\right) j_{\ell}\left(k_{2} r_{2}\right) j_{\ell^{\prime}}\left(k_{3} r_{3}\right) \sqrt{\ell(\ell+1)}\left(\ell^{\prime}\left(\ell^{\prime}+1\right)\right)^{3 / 2} .(\mathrm{A} .8
\end{aligned}
$$

From which the reduced bispectrum is given by

$$
b_{\ell_{1} \ell_{2} \ell_{3}}^{\nabla \kappa \nabla \psi}=\frac{4}{\pi^{2}} A_{\ell_{1} \ell_{2} \ell_{3}} Z_{\ell_{2} \ell_{3}}^{\nabla_{b} \kappa \nabla^{b} \psi}\left(z_{1}, z_{2}, z_{3}\right)+\text { perm. . }
$$

In terms of power spectra this leads to

$$
\begin{aligned}
b_{\ell_{1} \ell_{2} \ell_{3}}^{\nabla \kappa \nabla}= & A_{\ell_{1} \ell_{2} \ell_{3}}\left(\sqrt{\frac{\ell_{2}\left(\ell_{2}+1\right)}{\ell_{3}\left(\ell_{3}+1\right)}} c_{\ell_{2}}^{\kappa \delta}\left(z_{1}, z_{2}\right) c_{\ell_{3}}^{\kappa \delta}\left(z_{1}, z_{3}\right)+\sqrt{\frac{\ell_{3}\left(\ell_{3}+1\right)}{\ell_{2}\left(\ell_{2}+1\right)}} c_{\ell_{3}}^{\kappa \delta}\left(z_{1}, z_{3}\right) c_{\ell_{2}}^{\kappa \delta}\left(z_{1}, z_{2}\right)\right) \\
& +A_{\ell_{2} \ell_{1} \ell_{3}}\left(\sqrt{\frac{\ell_{1}\left(\ell_{1}+1\right)}{\ell_{3}\left(\ell_{3}+1\right)}} c_{\ell_{1}}^{\kappa \delta}\left(z_{2}, z_{1}\right) c_{\ell_{3}}^{\kappa \delta}\left(z_{2}, z_{3}\right)+\sqrt{\frac{\ell_{3}\left(\ell_{3}+1\right)}{\ell_{1}\left(\ell_{1}+1\right)}} c_{\ell_{3}}^{\kappa \delta}\left(z_{2}, z_{3}\right) c_{\ell_{1}}^{\kappa \delta}\left(z_{2}, z_{1}\right)\right) \\
& +A_{\ell_{3} \ell_{1} \ell_{2}}\left(\sqrt{\frac{\ell_{1}\left(\ell_{1}+1\right)}{\ell_{2}\left(\ell_{2}+1\right)}} c_{\ell_{1}}^{\kappa \delta}\left(z_{3}, z_{1}\right) c_{\ell_{2}}^{\kappa \delta}\left(z_{3}, z_{2}\right)+\sqrt{\frac{\ell_{2}\left(\ell_{2}+1\right)}{\ell_{1}\left(\ell_{1}+1\right)}} c_{\ell_{2}}^{\kappa \delta}\left(z_{3}, z_{2}\right) c_{\ell_{1}}^{\kappa \delta}\left(z_{3}, z_{1}\right)\right) \\
& =A_{\ell_{1} \ell_{2} \ell_{3}} \frac{\ell_{2}\left(\ell_{2}+1\right)+\ell_{3}\left(\ell_{3}+1\right)}{\sqrt{\ell_{2}\left(\ell_{2}+1\right) \ell_{3}\left(\ell_{3}+1\right)}} c_{\ell_{2}}^{\kappa \delta}\left(z_{1}, z_{2}\right) c_{\ell_{3}}^{\kappa \delta}\left(z_{1}, z_{3}\right) \\
& +A_{\ell_{2} \ell_{1} \ell_{3}} \frac{\ell_{1}\left(\ell_{1}+1\right)+\ell_{3}\left(\ell_{3}+1\right)}{\sqrt{\ell_{1}\left(\ell_{1}+1\right) \ell_{3}\left(\ell_{3}+1\right)}} c_{\ell_{1}}^{\kappa \delta}\left(z_{2}, z_{1}\right) c_{\ell_{3}}^{\kappa \delta}\left(z_{2}, z_{3}\right) \\
& +A_{\ell_{3} \ell_{1} \ell_{2}} \frac{\ell_{1}\left(\ell_{1}+1\right)+\ell_{2}\left(\ell_{2}+1\right)}{\sqrt{\ell_{1}\left(\ell_{1}+1\right) \ell_{2}\left(\ell_{2}+1\right)}} c_{\ell_{1}}^{\kappa \delta}\left(z_{3}, z_{1}\right) c_{\ell_{2}}^{\kappa \delta}\left(z_{3}, z_{2}\right) .
\end{aligned}
$$

\section{A.12 Term $\int_{0}^{r(z)} \frac{d r}{r} \nabla^{b} \kappa \nabla_{b} \Psi_{1}$}

We want to compute

$$
\left\langle\left(-2 \int_{0}^{r_{1}} \frac{d r}{r} \nabla^{b} \kappa \nabla_{b} \Psi_{1}\right)\left(\mathbf{n}_{1}, z_{1}\right) \delta\left(\mathbf{n}_{2}, z_{2}\right) \delta\left(\mathbf{n}_{3}, z_{3}\right)\right\rangle_{c}+\text { perm. } .
$$


Expressing the perturbation variables in Fourier space, we find

$$
\begin{aligned}
\langle\cdots\rangle= & -\frac{1}{(2 \pi)^{12}} \int_{0}^{r_{1}} \frac{d r}{r^{2}} \int d^{3} k d^{3} k_{1} d^{3} k_{2} d^{3} k_{3} \int_{0}^{r} d r^{\prime} \frac{r-r^{\prime}}{r r^{\prime}} \int_{0}^{r} d r^{\prime \prime} \\
& \times \frac{1}{2}\left(\not \partial^{*} \Delta_{2} e^{i \mathbf{k} \cdot \mathbf{n}_{1} r^{\prime}} \not \partial e^{i \mathbf{k}_{1} \cdot \mathbf{n}_{1} r^{\prime \prime}}+\not \Delta_{2} e^{i \mathbf{k} \cdot \mathbf{n}_{1} r^{\prime}} \not \partial^{*} e^{i \mathbf{k}_{1} \cdot \mathbf{n}_{1} r^{\prime \prime}}\right) e^{i\left(\mathbf{k}_{2} \cdot \mathbf{n}_{2} r_{2}+\mathbf{k}_{3} \cdot \mathbf{n}_{3} r_{3}\right)} \\
& \times T_{\Psi+\Phi}\left(k, \eta^{\prime}\right) T_{\Psi+\Phi}\left(k_{1}, \eta^{\prime \prime}\right) T_{\delta}\left(k_{2}, \eta_{2}\right) T_{\delta}\left(k_{3}, \eta_{3}\right)\left\langle R(\mathbf{k}) R\left(\mathbf{k}_{1}\right) R\left(\mathbf{k}_{2}\right) R\left(\mathbf{k}_{3}\right)\right\rangle \\
= & -\frac{1}{(2 \pi)^{6}} \int_{0}^{r_{1}} \frac{d r}{r^{2}} \int d^{3} k_{2} d^{3} k_{3} \int_{0}^{r} d r^{\prime} \frac{r-r^{\prime}}{r r^{\prime}} \int_{0}^{r} d r^{\prime \prime} \\
& \times \frac{1}{2}\left(\not \partial^{*} \Delta_{2} e^{-i \mathbf{k}_{2} \cdot \mathbf{n}_{1} r^{\prime}} \not \partial e^{-i \mathbf{k}_{3} \cdot \mathbf{n}_{1} r^{\prime \prime}}+\not \partial \Delta_{2} e^{-i \mathbf{k}_{2} \cdot \mathbf{n}_{1} r^{\prime}} \not \partial^{*} e^{-i \mathbf{k}_{3} \cdot \mathbf{n}_{1} r^{\prime \prime}}\right) e^{i\left(\mathbf{k}_{2} \cdot \mathbf{n}_{2} r_{2}+\mathbf{k}_{3} \cdot \mathbf{n}_{3} r_{3}\right)} \\
& \times T_{\Psi+\Phi}\left(k_{2}, \eta^{\prime}\right) T_{\Psi+\Phi}\left(k_{3}, \eta^{\prime \prime}\right) T_{\delta}\left(k_{2}, \eta_{2}\right) T_{\delta}\left(k_{3}, \eta_{3}\right) P\left(k_{2}\right) P_{R}\left(k_{3}\right) \\
& -\frac{1}{(2 \pi)^{6}} \int_{0}^{r_{1}} \frac{d r}{r^{2}} \int d^{3} k_{2} d^{3} k_{3} \int_{0}^{r} d r^{\prime} \frac{r-r^{\prime}}{r r^{\prime}} \int_{0}^{r} d r^{\prime \prime} \\
& \times \frac{1}{2}\left(\not \partial^{*} \Delta_{2} e^{-i \mathbf{k}_{3} \cdot \mathbf{n}_{1} r^{\prime}} \not \partial e^{-i \mathbf{k}_{2} \cdot \mathbf{n}_{1} r^{\prime \prime}}+\not \partial \Delta_{2} e^{-i \mathbf{k}_{3} \cdot \mathbf{n}_{1} r^{\prime}} \not \partial^{*} e^{-i \mathbf{k}_{2} \cdot \mathbf{n}_{1} r^{\prime \prime}}\right) e^{i\left(\mathbf{k}_{2} \cdot \mathbf{n}_{2} r_{2}+\mathbf{k}_{3} \cdot \mathbf{n}_{3} r_{3}\right)} \\
& \times T_{\Psi+\Phi}\left(k_{3}, \eta^{\prime}\right) T_{\Psi+\Phi}\left(k_{2}, \eta^{\prime \prime}\right) T_{\delta}\left(k_{2}, \eta_{2}\right) T_{\delta}\left(k_{3}, \eta_{3}\right) P\left(k_{2}\right) P_{R}\left(k_{3}\right) .
\end{aligned}
$$

With this we obtain the following expression for the bispectrum

$$
\begin{aligned}
& B_{0}^{\int_{0}^{r_{1}} \frac{d r}{r} \nabla^{b} \kappa \nabla_{b} \Psi_{1}}\left(\mathbf{n}_{1}, \mathbf{n}_{2}, \mathbf{n}_{3}, z_{1}, z_{2}, z_{3}\right) \\
& =\frac{4}{\pi^{2}} \sum_{\substack{\ell, \ell^{\prime} \\
m, m^{\prime}}} \frac{1}{2}\left[-1 Y_{\ell m}\left(\mathbf{n}_{1}\right)_{1} Y_{\ell^{\prime} m^{\prime}}\left(\mathbf{n}_{1}\right)+{ }_{1} Y_{\ell m}\left(\mathbf{n}_{1}\right)_{-1} Y_{\ell^{\prime} m^{\prime}}\left(\mathbf{n}_{1}\right)\right] Y_{\ell m}^{*}\left(\mathbf{n}_{2}\right) Y_{\ell^{\prime} m^{\prime}}^{*}\left(\mathbf{n}_{3}\right) \\
& \quad \times Z_{\ell \ell^{\prime}}^{\int_{0}^{r_{1}} \frac{d r}{r} \nabla^{b} \kappa \nabla_{b} \Psi_{1}}\left(z_{1}, z_{2}, z_{3}\right)+\text { perm. }
\end{aligned}
$$

where

$$
\begin{aligned}
Z_{\ell \ell^{\prime}}^{\int_{0}^{r_{1}} \frac{d r}{r} \nabla^{b} \kappa \nabla_{b} \Psi_{1}}\left(z_{1}, z_{2}, z_{3}\right)=-\int_{0}^{r_{1}} \frac{d r}{r^{2}} \int d k_{2} k_{2}^{2} d k_{3} k_{3}^{2} \int_{0}^{r} d r^{\prime} \frac{r-r^{\prime}}{r r^{\prime}} \int_{0}^{r} d r^{\prime \prime} \\
\times T_{\Psi+\Phi}\left(k_{2}, \eta^{\prime}\right) T_{\Psi+\Phi}\left(k_{3}, \eta^{\prime \prime}\right) T_{\delta}\left(k_{2}, \eta_{2}\right) T_{\delta}\left(k_{3}, \eta_{3}\right) P_{R}\left(k_{2}\right) P_{R}\left(k_{3}\right) \\
\times(\ell(\ell+1))^{3 / 2} \sqrt{\ell^{\prime}\left(\ell^{\prime}+1\right)} j_{\ell}\left(k_{2} r^{\prime}\right) j_{\ell^{\prime}}\left(k_{3} r^{\prime \prime}\right) j_{\ell}\left(k_{2} r_{2}\right) j_{\ell^{\prime}}\left(k_{3} r_{3}\right) \\
\quad-\int_{0}^{r_{1}} \frac{d r}{r^{2}} \int d k_{2} k_{2}^{2} d k_{3} k_{3}^{2} \int_{0}^{r} d r^{\prime} \frac{r-r^{\prime}}{r r^{\prime}} \int_{0}^{r} d r^{\prime \prime} \\
\quad \times T_{\Psi+\Phi}\left(k_{3}, \eta^{\prime}\right) T_{\Psi+\Phi}\left(k_{2}, \eta^{\prime \prime}\right) T_{\delta}\left(k_{2}, \eta_{2}\right) T_{\delta}\left(k_{3}, \eta_{3}\right) P_{R}\left(k_{2}\right) P_{R}\left(k_{3}\right) \\
\times \sqrt{\ell(\ell+1)}\left(\ell^{\prime}\left(\ell^{\prime}+1\right)\right)^{3 / 2} j_{\ell}\left(k_{2} r^{\prime \prime}\right) j \ell_{\ell^{\prime}}\left(k_{3} r^{\prime}\right) j_{\ell}\left(k_{2} r_{2}\right) j_{\ell^{\prime}}\left(k_{3} r_{3}\right) .
\end{aligned}
$$

Inserting this above, we obtain the reduced bispectrum

$$
b_{\ell_{1} \ell_{2} \ell_{3}}^{\int \nabla \kappa \nabla \Psi_{1}}=\frac{4}{\pi^{2}} A_{\ell_{1} \ell_{2} \ell_{3}} Z_{\ell_{2} \ell_{3}}^{\int_{0}^{r_{1}} \frac{d r}{r} \nabla^{b} \kappa \nabla_{b} \Psi_{1}}\left(z_{1}, z_{2}, z_{3}\right)+\text { perm. . }
$$


To express this in terms of angular power spectra, we define an additional transfer function in multipole space (3.31). With this we can rewrite the reduced bispectrum as follows

$$
\begin{aligned}
b_{\ell_{1} \ell_{2} \ell_{3}}^{\int \nabla \kappa \Psi_{1}}= & -A_{\ell_{1} \ell_{2} \ell_{3}} \sqrt{\ell_{2}\left(\ell_{2}+1\right)} \sqrt{\ell_{3}\left(\ell_{3}+1\right)} \\
& \times \int_{0}^{r_{1}} \frac{d r}{r}\left[c_{\ell_{2}}^{\kappa \delta}\left(z, z_{2}\right) c_{\ell_{3}}^{\Psi_{1} \delta}\left(z, z_{3}\right)+c_{\ell_{3}}^{\kappa \delta}\left(z, z_{3}\right) c_{\ell_{2}}^{\Psi_{1} \delta}\left(z, z_{2}\right)\right] \\
& -A_{\ell_{2} \ell_{1} \ell_{3}} \sqrt{\ell_{1}\left(\ell_{1}+1\right)} \sqrt{\ell_{3}\left(\ell_{3}+1\right)} \\
& \times \int_{0}^{r_{2}} \frac{d r}{r}\left[c_{\ell_{1}}^{\kappa \delta}\left(z, z_{1}\right) c_{\ell_{3}}^{\Psi_{1} \delta}\left(z, z_{3}\right)+c_{\ell_{3}}^{\kappa \delta}\left(z, z_{3}\right) c_{\ell_{1}}^{\Psi_{1} \delta}\left(z, z_{1}\right)\right] \\
& -A_{\ell_{3} \ell_{1} \ell_{2}} \sqrt{\ell_{1}\left(\ell_{1}+1\right)} \sqrt{\ell_{2}\left(\ell_{2}+1\right)} \\
& \times \int_{0}^{r_{3}} \frac{d r}{r}\left[c_{\ell_{1}}^{\kappa \delta}\left(z, z_{1}\right) c_{\ell_{2}}^{\Psi_{1} \delta}\left(z, z_{2}\right) c_{\ell_{2}}^{\kappa \delta}\left(z, z_{2}\right) c_{\ell_{1}}^{\Psi_{1} \delta}\left(z, z_{1}\right)\right],
\end{aligned}
$$

where $z=z(r)$ everywhere.

To include this and the following terms in the configuration with window functions we apply Limber approximation. Using the Limber approximation for the $k_{2}$ and $k_{3}$ integrals of Eq. (A.92) we obtain

$$
\begin{aligned}
& {\left[Z_{\ell \ell^{\prime}}^{\int_{0}^{r_{1}} \frac{d r}{r} \nabla^{b} \kappa \nabla_{b} \Psi_{1}}\left(z_{1}, z_{2}, z_{3}\right)\right]^{\text {(Limber) }}=-\frac{\pi^{2}}{4} \int_{0}^{r_{1}} \frac{d r}{r^{2}} \frac{r-r_{2}}{r r_{2}} \Theta\left(r-r_{2}\right) \Theta\left(r-r_{3}\right)} \\
& \times \frac{1}{r_{2}^{2} r_{3}^{2}} P_{R}\left(\frac{\ell+1 / 2}{r_{2}}\right) P_{R}\left(\frac{\ell^{\prime}+1 / 2}{r_{3}}\right) T_{\Psi+\Phi}\left(\frac{\ell+1 / 2}{r_{2}}, \eta_{2}\right) T_{\Psi+\Phi}\left(\frac{\ell^{\prime}+1 / 2}{r_{3}}, \eta_{3}\right) \\
& \times T_{\delta}\left(\frac{\ell+1 / 2}{r_{2}}, \eta_{2}\right) T_{\delta}\left(\frac{\ell^{\prime}+1 / 2}{r_{3}}, \eta_{3}\right)[\ell(\ell+1)]^{3 / 2} \sqrt{\ell^{\prime}\left(\ell^{\prime}+1\right)} \\
& -\frac{\pi^{2}}{4} \int_{0}^{r_{1}} \frac{d r}{r^{2}} \frac{r-r_{3}}{r r_{3}} \Theta\left(r-r_{2}\right) \Theta\left(r-r_{3}\right) \frac{1}{r_{2}^{2} r_{3}^{2}} P_{R}\left(\frac{\ell+1 / 2}{r_{2}}\right) P_{R}\left(\frac{\ell^{\prime}+1 / 2}{r_{3}}\right) \\
& \times T_{\Psi+\Phi}\left(\frac{\ell+1 / 2}{r_{2}}, \eta_{2}\right) T_{\Psi+\Phi}\left(\frac{\ell^{\prime}+1 / 2}{r_{3}}, \eta_{3}\right) \\
& \times T_{\delta}\left(\frac{\ell+1 / 2}{r_{2}}, \eta_{2}\right) T_{\delta}\left(\frac{\ell^{\prime}+1 / 2}{r_{3}}, \eta_{3}\right)\left[\ell^{\prime}\left(\ell^{\prime}+1\right)\right]^{3 / 2} \sqrt{\ell(\ell+1)} .
\end{aligned}
$$


The integral over $r$ can now be performed analytically, and we are finally left with

$$
\begin{aligned}
& {\left[Z_{\ell \ell^{\prime}}^{\int_{0}^{r_{1}} \frac{d r}{r} \nabla^{b} \kappa \nabla_{b} \Psi_{1}}\left(z_{1}, z_{2}, z_{3}\right)\right]^{(\text {Limber })}=-\Theta\left(z_{1}-z_{2}\right) \Theta\left(z_{2}-z_{3}\right) \frac{\pi^{2}}{4} \frac{1}{r_{2}^{2} r_{3}^{2}}} \\
& \times P_{R}\left(\frac{\ell+1 / 2}{r_{2}}\right) P_{R}\left(\frac{\ell^{\prime}+1 / 2}{r_{3}}\right) T_{\Psi+\Phi}\left(\frac{\ell+1 / 2}{r_{2}}, \eta_{2}\right) T_{\Psi+\Phi}\left(\frac{\ell^{\prime}+1 / 2}{r_{3}}, \eta_{3}\right) \\
& \times T_{\delta}\left(\frac{\ell+1 / 2}{r_{2}}, \eta_{2}\right) T_{\delta}\left(\frac{\ell^{\prime}+1 / 2}{r_{3}}, \eta_{3}\right) \frac{\left(r_{1}-r_{2}\right)}{2 r_{1}^{2} r_{2}^{2}} \\
& \times\left[(\ell(\ell+1))^{3 / 2} \sqrt{\ell^{\prime}\left(\ell^{\prime}+1\right)}\left(r_{1}-r_{2}\right)+\left(\ell^{\prime}\left(\ell^{\prime}+1\right)\right)^{3 / 2} \sqrt{\ell(\ell+1)} \frac{2 r_{1} r_{2}-r_{3}\left(r_{1}+r_{2}\right)}{r_{3}}\right] \\
& -\Theta\left(z_{1}-z_{3}\right) \Theta\left(z_{3}-z_{2}\right) \frac{\pi^{2}}{4} \frac{1}{r_{2}^{2} r_{3}^{2}} \\
& \times P_{R}\left(\frac{\ell+1 / 2}{r_{2}}\right) P_{R}\left(\frac{\ell^{\prime}+1 / 2}{r_{3}}\right) T_{\Psi+\Phi}\left(\frac{\ell+1 / 2}{r_{2}}, \eta_{2}\right) T_{\Psi+\Phi}\left(\frac{\ell^{\prime}+1 / 2}{r_{3}}, \eta_{3}\right) \\
& \times T_{\delta}\left(\frac{\ell+1 / 2}{r_{2}}, \eta_{2}\right) T_{\delta}\left(\frac{\ell^{\prime}+1 / 2}{r_{3}}, \eta_{3}\right) \frac{\left(r_{1}-r_{3}\right)}{2 r_{1}^{2} r_{3}^{2}} \\
& \times\left[(\ell(\ell+1))^{3 / 2} \sqrt{\ell^{\prime}\left(\ell^{\prime}+1\right)} \frac{2 r_{1} r_{3}-r_{2}\left(r_{1}+r_{3}\right)}{r_{2}}+\left(\ell^{\prime}\left(\ell^{\prime}+1\right)\right)^{3 / 2} \sqrt{\ell(\ell+1)}\left(r_{1}-r_{3}\right)\right] .
\end{aligned}
$$

\section{A.13 Term $\int_{0}^{r_{s}} d r \frac{r_{s}-r}{r_{s} r} \Delta_{2}\left(\nabla^{b} \Psi_{1} \nabla_{b} \Psi_{1}\right)$}

It is convenient to split this term as follows

$$
\begin{aligned}
-\frac{1}{2} \int_{0}^{r_{s}} d r & \frac{r_{s}-r}{r_{s} r} \Delta_{2}\left(\nabla^{b} \Psi_{1} \nabla_{b} \Psi_{1}\right) \\
& =-\frac{1}{2} \int_{0}^{r_{s}} d r \frac{r_{s}-r}{r_{s} r}\left[2\left(\nabla^{b} \Delta_{2} \Psi_{1}\right) \nabla_{b} \Psi_{1}+2 \nabla^{a} \nabla^{b} \Psi_{1} \nabla_{a} \nabla_{b} \Psi_{1}\right] \\
& =-\frac{1}{2} \int_{0}^{r_{s}} d r \frac{r_{s}-r}{r_{s} r}\left[2\left(\nabla^{b} \Delta_{2} \Psi_{1}\right) \nabla_{b} \Psi_{1}+\not \partial^{* 2} \Psi_{1} \not \partial^{2} \Psi_{1}+\left(\Delta_{2} \Psi_{1}\right)^{2}\right]
\end{aligned}
$$

We want to compute the expectation value of the first of these terms

$$
\left\langle\left(-\int_{0}^{r} d r^{\prime} \frac{r-r^{\prime}}{r r^{\prime}}\left(\nabla^{b} \Delta_{2} \Psi_{1}\right) \nabla_{b} \Psi_{1}\right)\left(\mathbf{n}_{1}, z_{1}\right) \delta\left(\mathbf{n}_{2}, z_{2}\right) \delta\left(\mathbf{n}_{3}, z_{3}\right)\right\rangle_{c}+\text { perm. }
$$

We can use the results of section (A.11) finding the bispectrum

$$
\begin{aligned}
& B^{\int_{0}^{r_{s}} d r \frac{r_{s}-r}{r_{s} r}\left(\left(\nabla^{b} \Delta_{2} \Psi_{1}\right) \nabla_{b} \Psi_{1}\right)}\left(\mathbf{n}_{1}, \mathbf{n}_{2}, \mathbf{n}_{3}, z_{1}, z_{2}, z_{3}\right) \\
& =\frac{4}{\pi^{2}} \sum_{\substack{\ell, \ell^{\prime} \\
m, m^{\prime}}} \frac{1}{2}\left[{ }_{1} Y_{\ell m}\left(\mathbf{n}_{1}\right)_{1} Y_{\ell^{\prime} m^{\prime}}\left(\mathbf{n}_{1}\right)+{ }_{1} Y_{\ell m}\left(\mathbf{n}_{1}\right)_{-1} Y_{\ell^{\prime} m^{\prime}}\left(\mathbf{n}_{1}\right)\right] Y_{\ell m}^{*}\left(\mathbf{n}_{2}\right) Y_{\ell^{\prime} m^{\prime}}^{*}\left(\mathbf{n}_{3}\right) \\
& \times Z_{\ell \ell^{\prime}}^{\int_{0}^{r_{s}} d r \frac{r_{s}-r}{r_{s} r}\left(\nabla^{b} \Delta_{2} \Psi_{1}\right) \nabla_{b} \Psi_{1}}\left(z_{1}, z_{2}, z_{3}\right)+\text { perm. },
\end{aligned}
$$


where

$$
\begin{aligned}
Z_{\ell \ell^{\prime}}^{\int_{0}^{r_{s}} d r \frac{r_{s}-r}{r_{s} r}} & \left(\nabla^{b} \Delta_{2} \Psi_{1}\right) \nabla_{b} \Psi_{1}\left(z_{1}, z_{2}, z_{3}\right) \\
& =-\int_{0}^{r_{1}} d r \frac{\left(r_{1}-r\right)}{r_{1} r^{3}} \int d k_{2} k_{2}^{2} d k_{3} k_{3}^{2} \int_{0}^{r} d r^{\prime} \int_{0}^{r} d r^{\prime \prime} \\
& \times\left[T_{\Psi+\Phi}\left(k_{2}, \eta^{\prime}\right) T_{\Psi+\Phi}\left(k_{3}, \eta^{\prime \prime}\right) T_{\delta}\left(k_{2}, \eta_{2}\right) T_{\delta}\left(k_{3}, \eta_{3}\right) P_{R}\left(k_{2}\right) P_{R}\left(k_{3}\right)\right. \\
& \left.\times(\ell(\ell+1))^{3 / 2} \sqrt{\ell^{\prime}\left(\ell^{\prime}+1\right)} j_{\ell}\left(k_{2} r^{\prime}\right) j_{\ell^{\prime}}\left(k_{3} r^{\prime \prime}\right) j_{\ell}\left(k_{2} r_{2}\right) j_{\ell^{\prime}}\left(k_{3} r_{3}\right)\right] \\
& -\int_{0}^{r_{1}} d r \frac{\left(r_{1}-r\right)}{r_{1} r^{3}} \int d k_{2} k_{2}^{2} d k_{3} k_{3}^{2} \int_{0}^{r} d r^{\prime} \int_{0}^{r} d r^{\prime \prime} \\
& \times\left[T_{\Psi+\Phi}\left(k_{2}, \eta^{\prime \prime}\right) T_{\Psi+\Phi}\left(k_{3}, \eta^{\prime}\right) T_{\delta}\left(k_{2}, \eta_{2}\right) T_{\delta}\left(k_{3}, \eta_{3}\right) P_{R}\left(k_{2}\right) P_{R}\left(k_{3}\right)\right. \\
& \left.\times \sqrt{\ell(\ell+1)}\left(\ell^{\prime}\left(\ell^{\prime}+1\right)\right)^{3 / 2} j_{\ell}\left(k_{2} r^{\prime \prime}\right) j_{\ell^{\prime}}\left(k_{3} r^{\prime}\right) j_{\ell}\left(k_{2} r_{2}\right) j_{\ell^{\prime}}\left(k_{3} r_{3}\right)\right] .
\end{aligned}
$$

Again, the reduced bispectrum is

$$
b_{\ell_{1} \ell_{2} \ell_{3}}^{\int\left(\nabla \Delta_{2} \Psi_{1}\right) \nabla \Psi_{1}}=\frac{4}{\pi^{2}} A_{\ell_{1} \ell_{2} \ell_{3}} Z_{\ell_{2} \ell_{3}}^{\int_{0}^{r_{s}} d r \frac{r_{s}-r}{r_{s} r}\left(\nabla^{b} \Delta_{2} \Psi_{1}\right) \nabla_{b} \Psi_{1}}\left(z_{1}, z_{2}, z_{3}\right)+\text { perm. },
$$

and in terms of power spectra

$$
\begin{aligned}
b_{\ell_{1} \ell_{2} \ell_{3}}^{\int\left(\nabla \Delta_{2} \Psi_{1}\right) \nabla \Psi_{1}}= & -A_{\ell_{1} \ell_{2} \ell_{3}} \sqrt{\ell_{2}\left(\ell_{2}+1\right) \ell_{3}\left(\ell_{3}+1\right)}\left[\ell_{2}\left(\ell_{2}+1\right)+\ell_{3}\left(\ell_{3}+1\right)\right] \\
& \times \int_{0}^{r_{1}} d r \frac{r_{1}-r}{r r_{1}} c_{\ell_{2}}^{\Psi_{1} \delta}\left(z(r), z_{2}\right) c_{\ell_{3}}^{\Psi_{1} \delta}\left(z(r), z_{3}\right) \\
& -A_{\ell_{2} \ell_{1} \ell_{3}} \sqrt{\ell_{1}\left(\ell_{1}+1\right) \ell_{3}\left(\ell_{3}+1\right)}\left[\ell_{1}\left(\ell_{1}+1\right)+\ell_{3}\left(\ell_{3}+1\right)\right] \\
& \times \int_{0}^{r_{2}} d r \frac{r_{2}-r}{r r_{2}} c_{\ell_{2}}^{\Psi_{1} \delta}\left(z(r), z_{1}\right) c_{\ell_{3}}^{\Psi_{1} \delta}\left(z(r), z_{3}\right) \\
& -A_{\ell_{3} \ell_{1} \ell_{2}} \sqrt{\ell_{1}\left(\ell_{1}+1\right) \ell_{2}\left(\ell_{2}+1\right)}\left[\ell_{1}\left(\ell_{1}+1\right)+\ell_{2}\left(\ell_{2}+1\right)\right] \\
& \times \int_{0}^{r_{3}} d r \frac{r_{3}-r}{r r_{3}} c_{\ell_{2}}^{\Psi_{1} \delta}\left(z(r), z_{1}\right) c_{\ell_{3}}^{\Psi_{1} \delta}\left(z(r), z_{2}\right) .
\end{aligned}
$$

Next we consider the second term in Eq. (A.97),

$$
\left\langle\left(-\frac{1}{2} \int_{0}^{r} d r^{\prime} \frac{r-r^{\prime}}{r r^{\prime}} \not^{* 2} \Psi_{1} \not \partial^{2} \Psi_{1}\right)\left(\mathbf{n}_{1}, z_{1}\right) \delta\left(\mathbf{n}_{2}, z_{2}\right) \delta\left(\mathbf{n}_{3}, z_{3}\right)\right\rangle_{c}+\text { perm. . }
$$

From (A.65) and (A.66) it follows

$$
\begin{aligned}
\not \partial \not e^{i \mathbf{k} \cdot \mathbf{n} r} & =4 \pi \sum_{\ell m} i^{\ell} j_{\ell}(k r) \sqrt{\frac{(\ell+2) !}{(\ell-2) !}}{ }_{2} Y_{\ell m}(\mathbf{n}) Y_{\ell m}^{*}(\hat{\mathbf{k}}), \\
\not{ }^{*} \not \partial^{*} e^{i \mathbf{k} \cdot \mathbf{n} r} & =4 \pi \sum_{\ell m} i^{\ell} j_{\ell}(k r) \sqrt{\frac{(\ell+2) !}{(\ell-2) !}}-{ }_{2} Y_{\ell m}(\mathbf{n}) Y_{\ell m}^{*}(\hat{\mathbf{k}}) .
\end{aligned}
$$


With this we find

$$
\begin{aligned}
B^{\int_{0}^{r} d r^{\prime}} \frac{\frac{r-r^{\prime}}{r r^{\prime}} \not \phi^{* 2} \Psi_{1} \not \phi^{2} \Psi_{1}}{\left(\mathbf{n}_{1}, \mathbf{n}_{2}, \mathbf{n}_{3}, z_{1}, z_{2}, z_{3}\right)} \\
=\frac{4}{\pi^{2}} \sum_{\substack{\ell, \ell^{\prime} \\
m, m^{\prime}}} \frac{1}{2}\left[{ }_{2} Y_{\ell m}\left(\mathbf{n}_{1}\right){ }_{-2} Y_{\ell^{\prime} m^{\prime}}\left(\mathbf{n}_{1}\right)+{ }_{-2} Y_{\ell m}\left(\mathbf{n}_{1}\right){ }_{2} Y_{\ell^{\prime} m^{\prime}}\left(\mathbf{n}_{1}\right)\right] Y_{\ell m}^{*}\left(\mathbf{n}_{2}\right) Y_{\ell^{\prime} m^{\prime}}^{*}\left(\mathbf{n}_{3}\right) \\
\quad \times Z_{\ell \ell^{\prime}}^{\int_{0}^{r} d r^{\prime} \frac{r-r^{\prime}}{r r^{\prime}} \not \phi^{* 2} \Psi_{1} \not \phi^{2} \Psi_{1}}\left(z_{1}, z_{2}, z_{3}\right)+\text { perm. }
\end{aligned}
$$

where

$$
\begin{aligned}
& Z_{\ell \ell^{\prime}}^{\int_{0}^{r} d r^{\prime} \frac{r-r^{\prime}}{r r^{\prime}} \not \not^{2} \Psi_{1} \not \not^{2} \Psi_{1}}\left(z_{1}, z_{2}, z_{3}\right) \\
& =-\int_{0}^{r_{1}} d r \frac{r_{1}-r}{r^{3} r_{1}} \int d k_{2} k_{2}^{2} d k_{3} k_{3}^{2} \int_{0}^{r} d r^{\prime} \int_{0}^{r} d r^{\prime \prime} T_{\Psi+\Phi}\left(k_{2}, \eta^{\prime}\right) T_{\Psi+\Phi}\left(k_{3}, \eta^{\prime \prime}\right) T_{\delta}\left(k_{2}, \eta_{2}\right) T_{\delta}\left(k_{3}, \eta_{3}\right) \\
& \times P_{R}\left(k_{2}\right) P_{R}\left(k_{3}\right) j_{\ell}\left(k_{2} r^{\prime}\right) j_{\ell^{\prime}}\left(k_{3} r^{\prime \prime}\right) j_{\ell}\left(k_{2} r_{2}\right) j_{\ell^{\prime}}\left(k_{3} r_{3}\right) \sqrt{\frac{(\ell+2) !}{(\ell-2) !}} \sqrt{\frac{\left(\ell^{\prime}+2\right) !}{\left(\ell^{\prime}-2\right) !}} .
\end{aligned}
$$

We now define

$$
C_{\ell_{1} \ell_{2} \ell_{3}} \equiv \frac{1}{2} \frac{\left(\begin{array}{ccc}
\ell_{1} & \ell_{2} & \ell_{3} \\
0 & 2 & -2
\end{array}\right)+\left(\begin{array}{ccc}
\ell_{1} & \ell_{2} & \ell_{3} \\
0 & -2 & 2
\end{array}\right)}{\left(\begin{array}{ccc}
\ell_{1} & \ell_{2} & \ell_{3} \\
0 & 0 & 0
\end{array}\right)}
$$

With this, the reduced bispectrum of the second term is

$$
b_{\ell_{1} \ell_{2} \ell_{3}}^{\left(\int_{0}^{r} d r^{\prime} \frac{r-r^{\prime}}{r r^{\prime}} \not \not^{* 2} \Psi_{1} \not \not^{2} \Psi_{1}\right) \delta \delta}=\frac{4}{\pi^{2}} C_{\ell_{1} \ell_{2} \ell_{3}} Z_{\ell_{2} \ell_{3}}^{\int_{0}^{r} d r^{\prime} \frac{r-r^{\prime}}{r r^{\prime}} \not \not^{* 2} \Psi_{1} \not \phi^{2} \Psi_{1}}\left(z_{1}, z_{2}, z_{3}\right)+\text { perm. },
$$

and in terms of angular power spectra

$$
\begin{aligned}
b_{\ell_{1} \ell_{2} \ell_{3}}^{\int \frac{r-r^{\prime}}{r^{\prime}} \phi^{* 2} \Psi_{1} \not \phi^{2} \Psi_{1}}= & -C_{\ell_{1} \ell_{2} \ell_{3}} \sqrt{\frac{\left(\ell_{2}+2\right) !}{\left(\ell_{2}-2\right) !}} \sqrt{\frac{\left(\ell_{3}+2\right) !}{\left(\ell_{3}-2\right) !}} \int_{0}^{r_{1}} d r \frac{r_{1}-r}{r r_{1}} c_{\ell_{2}}^{\Psi_{1} \delta}\left(z, z_{2}\right) c_{\ell_{3}}^{\Psi_{1} \delta}\left(z, z_{3}\right) \\
& -C_{\ell_{2} \ell_{1} \ell_{3}} \sqrt{\frac{\left(\ell_{1}+2\right) !}{\left(\ell_{1}-2\right) !}} \sqrt{\frac{\left(\ell_{3}+2\right) !}{\left(\ell_{3}-2\right) !}} \int_{0}^{r_{2}} d r \frac{r_{2}-r}{r r_{2}} c_{\ell_{1}}^{\Psi_{1} \delta}\left(z, z_{1}\right) c_{\ell_{3}}^{\Psi_{1} \delta}\left(z, z_{3}\right) \\
& -C_{\ell_{3} \ell_{1} \ell_{2}} \sqrt{\frac{\left(\ell_{1}+2\right) !}{\left(\ell_{1}-2\right) !}} \sqrt{\frac{\left(\ell_{2}+2\right) !}{\left(\ell_{2}-2\right) !}} \int_{0}^{r_{3}} d r \frac{r_{3}-r}{r r_{3}} c_{\ell_{1}}^{\Psi_{1} \delta}\left(z, z_{1}\right) c_{\ell_{2}}^{\Psi_{1} \delta}\left(z, z_{2}\right) .
\end{aligned}
$$

Finally, we compute also the third term of Eq. (A.97),

$$
\left\langle\left(-\frac{1}{2} \int_{0}^{r} d r^{\prime} \frac{r-r^{\prime}}{r r^{\prime}}\left(\Delta_{2} \Psi_{1}\right)^{2}\right)\left(\mathbf{n}_{1}, z_{1}\right) \delta\left(\mathbf{n}_{2}, z_{2}\right) \delta\left(\mathbf{n}_{3}, z_{3}\right)\right\rangle_{c}+\text { perm. . }
$$

We then obtain

$$
b_{\ell_{1} \ell_{2} \ell_{3}}^{\int \frac{r-r^{\prime}}{r \prime^{\prime}}}\left(\Delta_{2} \Psi_{1}\right)^{2}=\frac{4}{\pi^{2}} Z_{\ell_{2} \ell_{3}}^{\int \frac{r-r^{\prime}}{r r^{\prime}}\left(\Delta_{2} \Psi_{1}\right)^{2}}
$$


with

$$
\begin{aligned}
& Z_{\ell \ell^{\prime}}^{\int \frac{r-r^{\prime}}{r r^{\prime}}\left(\Delta_{2} \Psi_{1}\right)^{2}}\left(z_{1}, z_{2}, z_{3}\right) \\
& =-\int_{0}^{r_{1}} d r \frac{r_{1}-r}{r^{3} r_{1}} \int d k_{2} k_{2}^{2} d k_{3} k_{3}^{3} \int_{0}^{r} d r^{\prime} \int_{0}^{r} d r^{\prime \prime} T_{\Psi+\Phi}\left(k_{2}, \eta^{\prime}\right) T_{\Psi+\Phi}\left(k_{3}, \eta^{\prime \prime}\right) T_{\delta}\left(k_{2}, \eta_{2}\right) T_{\delta}\left(k_{3}, \eta_{3}\right) \\
& \quad \times P_{R}\left(k_{2}\right) P_{R}\left(k_{3}\right) j_{\ell}\left(k_{2} r^{\prime}\right) j_{\ell^{\prime}}\left(k_{3} r^{\prime \prime}\right) j_{\ell}\left(k_{2} r_{2}\right) j_{\ell^{\prime}}\left(k_{3} r_{3}\right) \ell(\ell+1) \ell^{\prime}\left(\ell^{\prime}+1\right) .
\end{aligned}
$$

which leads directly to

$$
\begin{aligned}
b_{\ell_{1} \ell_{2} \ell_{3}}^{\int \frac{r-r^{\prime}}{r r^{\prime}}\left(\Delta_{2} \Psi_{1}\right)^{2}}= & -\ell_{2}\left(\ell_{2}+1\right) \ell_{3}\left(\ell_{3}+1\right) \int_{0}^{r_{1}} d r \frac{\left(r_{1}-r\right)}{r r_{1}} c_{\ell_{2}}^{\Psi_{1} \delta}\left(z, z_{2}\right) c_{\ell_{3}}^{\Psi_{1} \delta}\left(z, z_{3}\right) \\
& -\ell_{1}\left(\ell_{1}+1\right) \ell_{3}\left(\ell_{3}+1\right) \int_{0}^{r_{2}} d r \frac{\left(r_{2}-r\right)}{r r_{2}} c_{\ell_{2}}^{\Psi_{1} \delta}\left(z, z_{1}\right) c_{\ell_{3}}^{\Psi_{1} \delta}\left(z, z_{3}\right) \\
& -\ell_{1}\left(\ell_{1}+1\right) \ell_{2}\left(\ell_{2}+1\right) \int_{0}^{r_{3}} d r \frac{\left(r_{3}-r\right)}{r r_{3}} c_{\ell_{1}}^{\Psi_{1} \delta}\left(z, z_{1}\right) c_{\ell_{3}}^{\Psi_{1} \delta}\left(z, z_{3}\right)
\end{aligned}
$$

The sum of the bispectra given in Eqs. (A.102), (A.110) and (A.114) yields the second integrated lensing term $b_{\ell_{1} \ell_{2} \ell_{3}}^{\int} \Delta_{2}\left(\nabla \Psi_{1} \nabla \Psi_{1}\right)$, given in Eq. (3.39).

Let us now use the Limber approximation on Eqs. (A.100), (A.107) and (A.113) to simplify these results. After some manipulation we obtain

$$
\begin{aligned}
& {\left[Z_{\ell \ell^{\prime}}^{\int_{0}^{r_{s}} d r \frac{r_{s}-r}{r_{s} r}\left(\nabla^{b} \Delta_{2} \Psi_{1}\right) \nabla_{b} \Psi_{1}}\left(z_{1}, z_{2}, z_{3}\right)\right]^{(\text {Limber })}} \\
& =-\left[\Theta\left(z_{1}-z_{2}\right) \Theta\left(z_{2}-z_{3}\right) \frac{\left(r_{1}-r_{2}\right)^{2}}{2 r_{1}^{2} r_{2}^{2}}+\Theta\left(z_{1}-z_{3}\right) \Theta\left(z_{3}-z_{2}\right) \frac{\left(r_{1}-r_{3}\right)^{2}}{2 r_{1}^{2} r_{3}^{2}}\right] \\
& \times\left[\sqrt{\ell^{\prime}\left(\ell^{\prime}+1\right)}(\ell(\ell+1))^{3 / 2}+\sqrt{\ell(\ell+1)}\left(\ell^{\prime}\left(\ell^{\prime}+1\right)\right)^{3 / 2}\right] \frac{\pi^{2}}{4} \frac{1}{r_{2}^{2} r_{3}^{2}} \\
& \times P_{R}\left(\frac{\ell+1 / 2}{r_{2}}\right) P_{R}\left(\frac{\ell^{\prime}+1 / 2}{r_{3}}\right) T_{\Psi+\Phi}\left(\frac{\ell+1 / 2}{r_{2}}, \eta_{2}\right) T_{\Psi+\Phi}\left(\frac{\ell^{\prime}+1 / 2}{r_{3}}, \eta_{3}\right) \\
& \times T_{\delta}\left(\frac{\ell+1 / 2}{r_{2}}, \eta_{2}\right) T_{\delta}\left(\frac{\ell^{\prime}+1 / 2}{r_{3}}, \eta_{3}\right), \\
& {\left[Z_{\ell \ell^{\prime}}^{\int_{0}^{r} d r^{\prime} \frac{r-r^{\prime}}{r r^{\prime}} \not \not^{* 2} \Psi_{1} \not \not^{2} \Psi_{1}}\left(z_{1}, z_{2}, z_{3}\right)\right]^{(\text {Limber })}} \\
& =-\left[\Theta\left(z_{1}-z_{2}\right) \Theta\left(z_{2}-z_{3}\right) \frac{\left(r_{1}-r_{2}\right)^{2}}{2 r_{1}^{2} r_{2}^{2}}+\Theta\left(z_{1}-z_{3}\right) \Theta\left(z_{3}-z_{2}\right) \frac{\left(r_{1}-r_{3}\right)^{2}}{2 r_{1}^{2} r_{3}^{2}}\right] \\
& \times \sqrt{\frac{(\ell+2) !}{(\ell-2) !}} \sqrt{\frac{\left(\ell^{\prime}+2\right) !}{\left(\ell^{\prime}-2\right) !}} \frac{\pi^{2}}{4} \frac{1}{r_{2}^{2} r_{3}^{2}} \\
& \times P_{R}\left(\frac{\ell+1 / 2}{r_{2}}\right) P_{R}\left(\frac{\ell^{\prime}+1 / 2}{r_{3}}\right) T_{\Psi+\Phi}\left(\frac{\ell+1 / 2}{r_{2}}, \eta_{2}\right) T_{\Psi+\Phi}\left(\frac{\ell^{\prime}+1 / 2}{r_{3}}, \eta_{3}\right) \\
& \times T_{\delta}\left(\frac{\ell+1 / 2}{r_{2}}, \eta_{2}\right) T_{\delta}\left(\frac{\ell^{\prime}+1 / 2}{r_{3}}, \eta_{3}\right),
\end{aligned}
$$




$$
\begin{aligned}
& {\left[Z_{\ell \ell^{\prime}}^{\int \frac{r-r^{\prime}}{r r^{\prime}}\left(\Delta_{2} \Psi_{1}\right)^{2}}\left(z_{1}, z_{2}, z_{3}\right)\right]^{(\text {Limber })}} \\
& =-\left[\Theta\left(z_{1}-z_{2}\right) \Theta\left(z_{2}-z_{3}\right) \frac{\left(r_{1}-r_{2}\right)^{2}}{2 r_{1}^{2} r_{2}^{2}}+\Theta\left(z_{1}-z_{3}\right) \Theta\left(z_{3}-z_{2}\right) \frac{\left(r_{1}-r_{3}\right)^{2}}{2 r_{1}^{2} r_{3}^{2}}\right] \\
& \times \ell(\ell+1) \ell^{\prime}\left(\ell^{\prime}+1\right) \frac{\pi^{2}}{4} \frac{1}{r_{2}^{2} r_{3}^{2}} \\
& \times P_{R}\left(\frac{\ell+1 / 2}{r_{2}}\right) P_{R}\left(\frac{\ell^{\prime}+1 / 2}{r_{3}}\right) T_{\Psi+\Phi}\left(\frac{\ell+1 / 2}{r_{2}}, \eta_{2}\right) T_{\Psi+\Phi}\left(\frac{\ell^{\prime}+1 / 2}{r_{3}}, \eta_{3}\right) \\
& \times T_{\delta}\left(\frac{\ell+1 / 2}{r_{2}}, \eta_{2}\right) T_{\delta}\left(\frac{\ell^{\prime}+1 / 2}{r_{3}}, \eta_{3}\right) .
\end{aligned}
$$

\section{B Magnification Bias}

In an experiment the observed number of galaxies does not only depend on the true number of galaxies but also on their luminosity, since the experiment has a limiting sensitivity. It can therefore happen that an intrinsically less luminous galaxy which is magnified by foreground matter concentrations makes it into a survey whereas another intrinsically more luminous one which is de-magnified is below the flux limit of the experiment. This phenomenon, called magnification bias, is relevant as long as an experiment cannot see 'all galaxies'.

Denoting the number of galaxies per redshift bin and solid angle above a flux limit $F$ by $N(\mathbf{n}, z, F)$ and the number of galaxies with intrinsic luminosity $L$ by $N(\mathbf{n}, z, L)$, following [15], we have to second order

$$
\begin{gathered}
N(\mathbf{n}, z, \bar{F})=N(\mathbf{n}, z, F)+\frac{\partial}{\partial L} N(\mathbf{n}, z, \bar{L})\left(\delta L^{(1)}+\delta L^{(2)}\right)+\frac{1}{2} \frac{\partial^{2}}{\partial L^{2}} N(\mathbf{n}, z, \bar{L})\left(\delta L^{(1)}\right)^{2} \\
=N(\bar{z}, \bar{L})\left[1+\Delta^{(1)}+\Delta^{(2)}+\frac{\partial_{L} \bar{\rho}}{\bar{\rho}}\left(\delta L^{(1)}+\delta L^{(2)}\right)+\frac{1}{2} \frac{\partial_{L}^{2} \bar{\rho}}{\bar{\rho}}\left(\delta L^{(1)}\right)^{2}+\right. \\
\left.+\frac{\left(\partial_{L} \rho-\partial_{L} \bar{\rho}\right)^{(1)}}{\bar{\rho}} \delta L^{(1)}+\frac{\partial_{\eta}\left(\partial_{L} \bar{\rho}\right)}{\bar{\rho}} \delta L^{(1)} \frac{\delta z^{(1)}}{\mathcal{H}}\right]
\end{gathered}
$$

where $\delta z^{(1)}$ is the first order redshift perturbation given by (see [15])

$$
\delta z^{(1)}=-\partial_{r} v^{(1)}-\Psi^{(1)}-2 \int_{0}^{r(z)} d r \partial_{\eta} \Phi_{W}^{(1)} .
$$

Using that the flux is given by $F=L /\left(4 \pi d_{L}^{2}\right)=\bar{L} /\left(4 \pi \bar{d}_{L}^{2}\right)$, we have that at fixed flux the fluctuation of the intrinsic luminosity $L$ is given by the fluctuation of the luminosity distance squared. The perturbation of the luminosity distance to first order has been calculated in Ref. [52] and the result to second order can be found in [17-19]. Introducing also

$$
\begin{gathered}
\left(\frac{\partial \ln \bar{\rho}}{\partial \ln L}\right)(z, \bar{L})=-\frac{5}{2} s(z, \bar{L}), \quad \frac{\partial^{2}}{\partial(\ln L)^{2}}(\ln \bar{\rho})(z, \bar{L})=-\frac{5}{2} t(z, \bar{L}), \\
\left(1+\delta^{(1)}\right) \frac{\partial \ln \rho}{\partial \ln L}-\frac{\partial \ln \bar{\rho}}{\partial \ln L}=-\frac{5}{2}(\delta s)^{(1)}(z, \bar{L}),
\end{gathered}
$$


one obtains the following result to first order in perturbations (we neglect anisotropic stresses so that $\Phi^{(1)}=\Psi^{(1)}$, for the leading terms this make no difference)

$$
\begin{aligned}
\Delta^{(1)}(\mathbf{n}, z)= & \left(\frac{2-5 s}{\mathcal{H} r(z)}+5 s+\frac{\mathcal{H}^{\prime}}{\mathcal{H}^{2}}\right)\left(\partial_{r} v^{(1)}+\Psi^{(1)}+2 \int_{0}^{r(z)} d r \partial_{\eta} \Psi^{(1)}\right)+(5 s-1) \Psi^{(1)} \\
& +(2-5 s)\left(2 \Psi_{1}-\kappa^{(1)}\right)+\frac{1}{\mathcal{H}}\left(\partial_{\eta} \Psi^{(1)}+\partial_{r}^{2} v^{(1)}\right)+\delta^{(1)}
\end{aligned}
$$

This result has been originally derived in [14], see also [33] for details.

At second order one then obtains the following result for the leading contributions

$$
\begin{aligned}
\Sigma^{(2)}(\mathbf{n}, z)= & \delta^{(2)}+\mathcal{H}^{-1} \partial_{r}^{2} v^{(2)}-2\left(1-\frac{5}{2} s\right) \kappa^{(2)}+\mathcal{H}^{-2}\left[\left(\partial_{r}^{2} v\right)^{2}+\partial_{r} v \partial_{r}^{3} v\right] \\
& +\mathcal{H}^{-1}\left(\partial_{r} v \partial_{r} \delta+\partial_{r}^{2} v \delta\right)-2 \delta \kappa+5(\delta s)^{(1)} \kappa+\nabla_{a} \delta \nabla^{a} \psi \\
& +\mathcal{H}^{-1}\left[-2\left(1-\frac{5}{2} s\right) \partial_{r}^{2} v \kappa+\nabla_{a} \partial_{r}^{2} v \nabla^{a} \psi\right]+2\left(1-5 s+\frac{25}{4} s^{2}-\frac{5}{2} t\right) \kappa^{2} \\
& -2\left(1-\frac{5}{2} s\right) \nabla_{b} \kappa \nabla^{b} \psi-\left(1-\frac{5}{2} s\right) \frac{1}{2 r(z)} \int_{0}^{r(z)} d r \frac{r(z)-r}{r} \Delta_{2}\left(\nabla^{b} \Psi_{1} \nabla_{b} \Psi_{1}\right) \\
& -2\left(1-\frac{5}{2} s\right) \int_{0}^{r(z)} \frac{d r}{r^{2}} \nabla^{a} \Psi_{1} \nabla_{a} \kappa,
\end{aligned}
$$

If the number of galaxies depend on luminosity as a power law, $\rho \propto L^{p}$, Eqs. (B.4) and (B.5) imply $s=-2 p / 5, t=0$ and $(\delta s)^{(1)}=s \delta^{(1)}=-2 p \delta^{(1)} / 5$. It is interesting to note that the lensing terms vanish with $p=-1$, or equivalently $s=2 / 5$ at first order. Whereas, at second order there are non-vanishing lensing terms which contribute to the galaxy number counts. Indeed the terms $\nabla_{a} \delta \nabla^{a} \psi+\mathcal{H}^{-1} \nabla_{a} \partial_{r}^{2} v \nabla^{a} \psi$ are not affected by magnification bias.

\section{Intensity mapping}

In this section we show how to use the formalism developed in this work in the context of radio surveys measuring the intensity of the $21 \mathrm{~cm}$ emission line of neutral hydrogen, which can be used as a biased tracer of the dark matter field. This complementary observable does not resolve individual galaxies. It is based on the measurement of photon flux intensity, or equivalently on surface brightness temperature mapping.

To extend the calculation presented in [15] and in the current work we use the fact that, as shown in [53], the surface brightness temperature $T_{b}$ is directly proportional to the density distribution of the neutral atomic hydrogen $\Delta_{n_{\mathrm{HI}}}$ and inverse proportional to the square of the angular diameter distance, namely

$$
T_{b}(\mathbf{n}, z) \propto \frac{\Delta_{n_{\mathrm{HI}}}(\mathbf{n}, z)}{d_{A}^{2}(\mathbf{n}, z)}=\frac{\Delta_{n_{\mathrm{HI}}}(\mathbf{n}, z)}{(1+z)^{4} d_{L}^{2}(\mathbf{n}, z)} .
$$

Hence, we need consider the luminosity distance to second order [17-19] to compute the temperature fluctuations at the same order in perturbation theory. At first order one obtains [53]

$$
\Delta_{T}^{(1)}=\left(2+\frac{\mathcal{H}^{\prime}}{\mathcal{H}^{2}}\right)\left(\partial_{r} v^{(1)}+\Psi^{(1)}+2 \int_{0}^{r(z)} d r \partial_{\eta} \Psi^{(1)}\right)+\Psi^{(1)}+\frac{1}{\mathcal{H}}\left(\partial_{\eta} \Psi^{(1)}+\partial_{r}^{2} v^{(1)}\right)+\delta^{(1)} .
$$


(we have again neglected anisotropic stress), and at second order we find for the leading terms

$$
\begin{aligned}
\Sigma_{T}^{(2)}(\mathbf{n}, z)= & \delta^{(2)}+\mathcal{H}^{-1} \partial_{r}^{2} v^{(2)}+\mathcal{H}^{-2}\left[\left(\partial_{r}^{2} v\right)^{2}+\partial_{r} v \partial_{r}^{3} v\right] \\
& +\mathcal{H}^{-1}\left(\partial_{r} v \partial_{r} \delta+\partial_{r}^{2} v \delta\right)+\nabla_{a} \delta \nabla^{a} \psi+\mathcal{H}^{-1} \nabla_{a} \partial_{r}^{2} v \nabla^{a} \psi
\end{aligned}
$$

This expression agrees with [54]. Let us note that the result for 2nd order intensity mapping can be obtained from the one for galaxy number counts by simply setting $s=2 / 5,(\delta s)^{(1)}=$ $2 / 5 \delta^{(1)}$ and $t=0$. From Eqs. (C.2, C.3) it is evident that there is no lensing term for intensity mapping at first order (as a consequence of photons conservation), whereas there are non-vanishing lensing terms at second order. These terms, given by the product of the deflection angle $\nabla^{a} \psi$ with gradients of density and redshift space distortion, are analogous ones of those obtained when studying lensing of the cosmic microwave background (CMB), one just has to replace the temperature fluctuations, $\delta T / T$ by $\delta^{(1)}+\mathcal{H}^{-1} \partial_{r}^{2} v^{(1)}$. They simply stem from the fact that we have to evaluate the density and redshift space distortion in the unlensed direction $\mathbf{n}+\boldsymbol{\nabla} \psi$, see e.g. [51]. However, contrary to CMB analysis, when computing the second order power spectrum or the bispectrum the correlations between the deflection angles and density fluctuations can not be neglected in $\mathrm{HI}$ intensity mapping.

\section{References}

[1] Planck Collaboration, R. Adam et al., Planck 2015 results. I. Overview of products and scientific results, arXiv:1502.01582.

[2] Planck Collaboration, P. Ade et al., Planck 2015 results. XIII. Cosmological parameters, arXiv: 1502.01589.

[3] L. Samushia, B. A. Reid, M. White, W. J. Percival, A. J. Cuesta, et al., The Clustering of Galaxies in the SDSS-III Baryon Oscillation Spectroscopic Survey (BOSS): measuring growth rate and geometry with anisotropic clustering, Mon.Not.Roy.Astron.Soc. 439 (2014) 3504-3519, [arXiv: 1312.4899].

[4] BOSS Collaboration Collaboration, T. Delubac et al., Baryon Acoustic Oscillations in the Ly-alpha forest of BOSS DR11 quasars, arXiv:1404.1801.

[5] J. Frieman, Probing the accelerating universe, Phys.Today 67 (2014), no. 4 28-33.

[6] Euclid Theory Working Group Collaboration, L. Amendola et al., Cosmology and fundamental physics with the Euclid satellite, Living Rev.Rel. 16 (2013) 6, [arXiv:1206.1225].

[7] Cosmology SWG Collaboration, F. B. Abdalla et al., Cosmology from HI galaxy surveys with the $S K A$, arXiv: 1501.04035.

[8] J. M. Maldacena, Non-Gaussian features of primordial fluctuations in single field inflationary models, JHEP 0305 (2003) 013, [astro-ph/0210603].

[9] J. M. Maldacena and G. L. Pimentel, On graviton non-Gaussianities during inflation, JHEP 09 (2011) 045, [arXiv:1104.2846].

[10] F. Bernardeau, S. Colombi, E. Gaztanaga, and R. Scoccimarro, Large scale structure of the universe and cosmological perturbation theory, Phys.Rept. 367 (2002) 1-248, [astro-ph/0112551].

[11] J. Yoo, A. L. Fitzpatrick, and M. Zaldarriaga, A New Perspective on Galaxy Clustering as a Cosmological Probe: General Relativistic Effects, Phys.Rev. D80 (2009) 083514, [arXiv:0907.0707]. 
[12] J. Yoo, General Relativistic Description of the Observed Galaxy Power Spectrum: Do We Understand What We Measure?, Phys.Rev. D82 (2010) 083508, [arXiv:1009.3021].

[13] C. Bonvin and R. Durrer, What galaxy surveys really measure, Phys.Rev. D84 (2011) 063505, [arXiv: 1105.5280].

[14] A. Challinor and A. Lewis, The linear power spectrum of observed source number counts, Phys.Rev. D84 (2011) 043516, [arXiv: 1105.5292].

[15] E. Di Dio, R. Durrer, G. Marozzi, and F. Montanari, Galaxy number counts to second order and their bispectrum, JCAP 1412 (2014) 017, [arXiv:1407.0376]. [Erratum: JCAP1506,no.06,E01(2015)].

[16] M. Gasperini, G. Marozzi, F. Nugier, and G. Veneziano, Light-cone averaging in cosmology: Formalism and applications, JCAP 1107 (2011) 008, [arXiv:1104.1167].

[17] I. Ben-Dayan, G. Marozzi, F. Nugier, and G. Veneziano, The second-order luminosity-redshift relation in a generic inhomogeneous cosmology, JCAP 1211 (2012) 045, [arXiv: 1209.4326].

[18] G. Fanizza, M. Gasperini, G. Marozzi, and G. Veneziano, An exact Jacobi map in the geodesic light-cone gauge, JCAP 1311 (2013) 019, [arXiv:1308.4935].

[19] G. Marozzi, The luminosity distance-redshift relation up to second order in the Poisson gauge with anisotropic stress, Class. Quant. Grav. 32 (2015), no. 4 045004, [arXiv:1406.1135]. [Corrigendum: Class. Quant. Grav.32,179501(2015)].

[20] D. Bertacca, R. Maartens, and C. Clarkson, Observed galaxy number counts on the lightcone up to second order: I. Main result, JCAP 1409 (2014), no. 09 037, [arXiv: 1405.4403].

[21] D. Bertacca, R. Maartens, and C. Clarkson, Observed galaxy number counts on the lightcone up to second order: II. Derivation, JCAP 1411 (2014), no. 11 013, [arXiv:1406.0319].

[22] J. Yoo and M. Zaldarriaga, Beyond the Linear-Order Relativistic Effect in Galaxy Clustering: Second-Order Gauge-Invariant Formalism, Phys.Rev. D90 (2014) 023513, [arXiv: 1406.4140].

[23] A. Kehagias, A. M. Dizgah, J. Noreña, H. Perrier, and A. Riotto, A Consistency Relation for the Observed Galaxy Bispectrum and the Local non-Gaussianity from Relativistic Corrections, JCAP 1508 (2015), no. 08 018, [arXiv:1503.04467].

[24] J. Yoo and J.-O. Gong, Relativistic effects and primordial non-Gaussianity in the matter density fluctuation, arXiv:1509.08466.

[25] P. Peebles, The Large-Scale Structure of the Universe. Princeton University Press, 1980.

[26] L. Anderson et al., The clustering of galaxies in the SDSS-III Baryon Oscillation Spectroscopic Survey: measuring $D_{A}$ and $H$ at $z=0.57$ from the baryon acoustic peak in the Data Release 9 spectroscopic Galaxy sample, Mon. Not. Roy. Astron. Soc. 439 (2014), no. 1 83-101, [arXiv: 1303.4666].

[27] F. Montanari and R. Durrer, Measuring the lensing potential with galaxy clustering, JCAP 1510 (2015), no. 10 070, [arXiv:1506.01369].

[28] C. Bonvin, C. Clarkson, R. Durrer, R. Maartens, and O. Umeh, Do we care about the distance to the CMB? Clarifying the impact of second-order lensing, JCAP 1506 (2015), no. 06050 , [arXiv: 1503.07831].

[29] G. Fanizza, M. Gasperini, G. Marozzi, and G. Veneziano, A new approach to the propagation of light-like signals in perturbed cosmological backgrounds, JCAP 1508 (2015), no. 08020 , [arXiv: 1506.02003].

[30] V. Acquaviva, N. Bartolo, S. Matarrese, and A. Riotto, Second order cosmological perturbations from inflation, Nucl.Phys. B667 (2003) 119-148, [astro-ph/0209156].

[31] M. Goroff, B. Grinstein, S. Rey, and M. B. Wise, Coupling of Modes of Cosmological Mass Density Fluctuations, Astrophys.J. 311 (1986) 6-14. 
[32] N. Bartolo, D. Bertacca, M. Bruni, K. Koyama, R. Maartens, et al., A relativistic signature in large-scale structure: Scale-dependent bias from single-field inflation, arXiv:1506.00915.

[33] E. Di Dio, F. Montanari, J. Lesgourgues, and R. Durrer, The CLASSgal code for Relativistic Cosmological Large Scale Structure, JCAP 1311 (2013) 044, [arXiv:1307.1459].

[34] D. Bertacca, Observed galaxy number counts on the lightcone up to second order: III. Magnification Bias, Class. Quant. Grav. 32 (2015), no. 19 195011, [arXiv:1409.2024].

[35] M. Abramowitz and I. Stegun, Handbook of Mathematical Functions. Dover Publications, New York, 9th printing ed., 1970.

[36] N. Kaiser, Weak gravitational lensing of distant galaxies, Astrophys. J. 388 (1992) 272.

[37] M. LoVerde and N. Afshordi, Extended Limber Approximation, Phys.Rev. D78 (2008) 123506, [arXiv:0809.5112].

[38] F. Bernardeau, C. Bonvin, N. Van de Rijt, and F. Vernizzi, Cosmic shear bispectrum from second-order perturbations in General Relativity, Phys.Rev. D86 (2012) 023001, [arXiv:1112.4430].

[39] I. Gradshteyn and R. E.M, Table of Integrals, Series, and Products. Academic Press, 2007.

[40] J. Adamek, R. Durrer, and V. Tansella, Lensing signals from Spin-2 perturbations, arXiv: 1510.01566.

[41] D. Blas, J. Lesgourgues, and T. Tram, The Cosmic Linear Anisotropy Solving System (CLASS) II: Approximation schemes, JCAP 1107 (2011) 034, [arXiv:1104.2933].

[42] E. Di Dio, F. Montanari, R. Durrer, and J. Lesgourgues, Cosmological Parameter Estimation with Large Scale Structure Observations, JCAP 1401 (2014) 042, [arXiv:1308.6186].

[43] P. Fosalba, M. Crocce, E. Gaztaaga, and F. J. Castander, The MICE grand challenge lightcone simulation I. Dark matter clustering, Mon. Not. Roy. Astron. Soc. 448 (2015), no. 4 2987-3000, [arXiv: 1312.1707].

[44] P. Fosalba, E. Gaztaaga, F. J. Castander, and M. Crocce, The MICE Grand Challenge light-cone simulation III. Galaxy lensing mocks from all-sky lensing maps, Mon. Not. Roy. Astron. Soc. 447 (2015), no. 2 1319-1332, [arXiv:1312.2947].

[45] J. Adamek, D. Daverio, R. Durrer, and M. Kunz, General relativity and cosmic structure formation, arXiv:1509.01699.

[46] J. Adamek, R. Durrer, and M. Kunz, N-body methods for relativistic cosmology, Class. Quant. Grav. 31 (2014), no. 23 234006, [arXiv: 1408.3352].

[47] D. N. Spergel and D. M. Goldberg, Microwave background bispectrum. 1. Basic formalism, Phys. Rev. D59 (1999) 103001, [astro-ph/9811252].

[48] D. Varshalovich, A. Moskalev, and Khersonskii, Quantum Theory of Angular Momentum. World Scientific Pub Co Inc, 1988.

[49] E. Komatsu, The pursuit of non-gaussian fluctuations in the cosmic microwave background. PhD thesis, Tohoku U., Astron. Inst., 2001. astro-ph/0206039.

[50] H. T. Johansson and C. Forssén, Fast and Accurate Evaluation of Wigner 3j, 6j, and 9j Symbols using Prime Factorisation and Multi-Word Integer Arithmetic, arXiv:1504.08329.

[51] R. Durrer, The Cosmic Microwave Background. Cambridge University Press, 2008.

[52] C. Bonvin, R. Durrer, and M. A. Gasparini, Fluctuations of the luminosity distance, Phys.Rev. D73 (2006) 023523, [astro-ph/0511183].

[53] A. Hall, C. Bonvin, and A. Challinor, Testing General Relativity with 21-cm intensity mapping, Phys.Rev. D87 (2013), no. 6 064026, [arXiv: 1212.0728]. 
[54] O. Umeh, R. Maartens, and M. Santos, Nonlinear modulation of the HI power spectrum on ultra-large scales. I, arXiv:1509.03786. 